\title{
DETERMINATION OF HUMAN POWERED HELICOPTER STABILITY CHARACTERISTICS USING MULTI-BODY SYSTEM SIMULATION TECHNIQUES
}

\author{
A Thesis \\ Presented to \\ the Faculty of California Polytechnic State University \\ San Luis Obispo
}

\author{
In Partial Fulfillment \\ of the Requirements for the Degree \\ Master of Science in Aerospace Engineering
}

by

Sean Brown

November 2012 
(C) 2012

Sean Brown

ALL RIGHTS RESERVED 


\section{COMMITTEE MEMBERSHIP}

TITLE: Determination of Human Powered Helicopter Stability characteristics using Multi-Body System Simulation techniques

AUTHOR:

Sean Brown

DATE SUBMITTED: November 2012

COMMITTEE CHAIR: $\quad$ Eric Mehiel, Ph.D.

COMMITTEE MEMBER: Rob McDonald, Ph.D.

COMmitTEE MEMBER: Kurt Colvin, Ph.D.

COMMITTEE MEMBER: Pete Muller 


\begin{abstract}
Determination of Human Powered Helicopter Stability characteristics using Multi-Body System Simulation techniques
\end{abstract}

Sean Brown

\begin{abstract}
Multi-Body System Simulation combined with System Identification was developed as a method for determining the stability characteristics of a human powered helicopter(HPH) configurations. HPH stability remains a key component for meeting competition requirements, but has not been properly treated. Traditional helicopter dynamic analysis is not suited to the HPH due to its low rotation speeds and light weight. Multi-Body System Simulation is able to generate dynamic response data for any HPH configuration. System identification and linear stability theory are used to determine the stability characteristics from the dynamic response. This thesis focuses on the method development and doesn't present any $\mathrm{HPH}$ analysis results.
\end{abstract}




\section{Acknowledgements}

First and foremost, I would like to thank my parents for putting me on the path to success, for pushing me to go to college and for being supportive while I was here. I would like to express my greatest appreciation to my advisor, Dr. Eric Mehiel, who provided insight and patience. I wish to acknowledge the help provided by Dr. McDonald, whose door open was always open. The assistance provided by my committee members, Dr. Kurt Colvin and Pete Muller, was greatly appreciated.

I would like to thank my Uncle Butch for encouraging me to pursue my Master's Degree. Finally, I would like to offer a special thanks to Lindsay for her endless support and patience. 


\section{Contents}

List of Tables $\quad$ ix

List of Figures $\quad$ X

Nomenclature xiii

1 Introduction 1

1.1 Competition . . . . . . . . . . . . . . . . . 1

1.2 Previous Work ..................... 4

1.3 Solution . . . . . . . . . . . . . . . . . . 11

1.4 Thesis Scope. . . . . . . . . . . . . . . . . 14

1.5 Thesis Outline . . . . . . . . . . . . . . . 15

$\begin{array}{lll}2 & \text { Theory } & 17\end{array}$

2.1 Helicopter Configurations . . . . . . . . . . . . . . 17

2.1.1 The Human-Powered Helicopter Configuration . . . . . . . 18

2.2 Helicopter Dynamics . . . . . . . . . . . . . . . . . . . 20

2.2.1 Rigid Body Motion . . . . . . . . . . . . . . 22

2.2 .2 Blade Flap . . . . . . . . . . . . . . . . . . . . 24

2.3 Multi-Body System Simulation _. . . . . . . . . . . . . 30

2.4 Aerodynamic Model . . . . . . . . . . . . . . . . . . 30

2.4.1 Methodology .................. 32

2.4.2 Flow effects ..................... 35

2.5 System Identification . . . . . . . . . . . . . . . . . . . 38

2.6 Stability . . . . . . . . . . . . . . . . . . . . . . . 39

2.7 Summary . . . . . . . . . . . . . . . . . 41 
3 Implementation $\quad 42$

3.1 SimMechanics Implementation . . . . . . . . . . . . . . . 43

3.2 Text User Interface . . . . . . . . . . . . . . . . . . . . . 46

3.3 System Identification . . . . . . . . . . . . . . . . . . . . 49

3.4 Aerodynamic Model . . . . . . . . . . . . . . . . . . . . . . . 49

3.4.1 Verification .................... 52

3.4.2 Validation ................... 53

3.5 Summary . . . . . . . . . . . . . . . 60

4 Results $\quad 62$

4.1 Time Responses . . . . . . . . . . . . . . . . . . . 68

4.1.1 Gamera: Base Configuration . . . . . . . . . . . . . 69

4.1.2 Gamera: Uneven Rotor Inertia . . . . . . . . . . . . . 72

4.1.3 Gamera: Uneven Rotor Mass . . . . . . . . . . . . . 72

4.1.4 Gamera: Uneven Rotor Pitch . . . . . . . . . . . . . 73

4.1.5 Gamera: Initial Angular Offset . . . . . . . . . . . . . . 74

4.1.6 Gamera: Initial Linear Velocity . . . . . . . . . . . 76

4.2 Parameter Studies . . . . . . . . . . . . . . . . 77

4.3 Summary . . . . . . . . . . . . . . . . . 80

5 Conclusion $\quad 82$

5.1 Summary . . . . . . . . . . . . . . . . 82

5.2 Future Work . . . . . . . . . . . . . . . . 85

5.2.1 Model Development............... 85

$\begin{array}{ll}\text { Bibliography } & 87\end{array}$

A Custom Library Blocks $\quad 93$

A.1 Aerodynamic Model . . . . . . . . . . . . . . . . . . . 94

A.2 Force actuated joint . . . . . . . . . . . . . . . 95

B How to's $\quad 96$

B.1 Build a Compliant SimMechanics Model . . . . . . . . . . . . . . 99

B.2 Use the Model Discovery Class . . . . . . . . . . . . . . . . . . . . 101

B.3 Generate Time Response Plots . . . . . . . . . . . . . . . . . 102 
B.4 Add Structural Model . . . . . . . . . . . . . . . . . . . . . 103

B.5 Add Powerplant Model . . . . . . . . . . . . . . . . . . . . 105 


\section{List of Tables}

2.1 Helicopter Characteristics . . . . . . . . . . . . . . 19

3.1 Discretization Error: Richardson Extrapolation . . . . . . . . 53

3.2 Experimental Test Description . . . . . . . . . . . . . 54

4.1 Quadrotor Baseline configuration characteristics . . . . . . . 68

B.1 Coaxial Helicopter Characteristics . . . . . . . . . . . . . . . 98

B.2 SimMechanics Coaxial Model characteristics . . . . . . . . . . . 99 


\section{List of Figures}

1.1 Gamera II Quadrotor configuration, $[1]$. . . . . . . . . . . 2

1.2 Yuri I quadrotor configuration, $[2] \ldots \ldots$. . . . . . . . . 2

1.3 Gamera II's Highest flight, $[3]$. . . . . . . . . . . . . . . . 3

1.4 Gamera Triangular Truss Structure, $[3]$. . . . . . . . . . . . 6

1.5 Phase I Preliminary Design of Daedalus Project, $[4]$. . . . . . . . 6

1.6 Human Powered Ornithopter, Snowbird,[4] . . . . . . . . . . . 7

1.7 The VK-1 single main rotor with twin tails \& winglets [5] . . . . 8

1.8 Tip-Mounted Control system for Da Vinci II $[6]$. . . . . . . . . . . 9

1.9 Da Vinci configuration[6] . . . . . . . . . . . . . . . . . 10

1.10 Multi-body Simulation Method . . . . . . . . . . . . . . . 13

2.1 Lynx DRA Research Helicopter . . . . . . . . . . . . . . 18

2.2 Hinged main rotor degrees of freedom, [7] . . . . . . . . . . . . 21

2.3 Helicopter Swashplate mechanism, [7] . . . . . . . . . . . . 21

2.4 Flapping hinge coordinate system definition . . . . . . . . . . 26

2.5 Two Dimensional Aerodynamic Model . . . . . . . . . . . . . . . 28

2.6 Ground Effect Data from UMD along with the corresponding trendline . . . . . . . . . . . . . . . . . 38

3.1 Double Pendulum Drawing $[8]$. . . . . . . . . . . . . . 45

3.2 Double Pendulum Model[8] . . . . . . . . . . . . . . . . . 46

3.3 Class Diagram . . . . . . . . . . . . . . . . . . . . 48

3.4 Aerodynamic model implemented in Simulink . . . . . . . . . . 51 
3.5 Grid Refinement Study for Aerodynamic model on representative

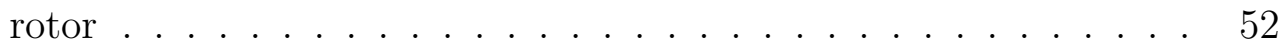

3.6 SimMechanics Validation using UMD GETR data $\Omega=54 \mathrm{rpm} \quad z / R=$

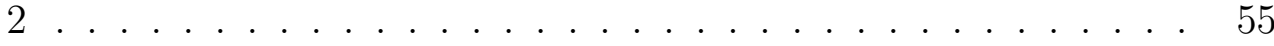

3.7 SimMechanics Validation using UMD GETR data $\Omega=66 \mathrm{rpm} \quad z / R=$ $2 \ldots \ldots \ldots \ldots \ldots \ldots \ldots \ldots$

3.8 SimMechanics Validation using UMD GETR data $\Omega=78 \mathrm{rpm} \quad z / R=$ $2 \ldots \ldots \ldots \ldots \ldots \ldots \ldots \ldots \ldots$

3.9 SimMechanics Validation using UMD GETR data $\Omega=82 \mathrm{rpm} \quad z / R=$ $2 \ldots \ldots \ldots \ldots \ldots \ldots \ldots \ldots$

3.10 SimMechanics Validation using UMD FSTR data $\Omega=18 \mathrm{rpm} \quad z / R=$ $.1 \ldots \ldots \ldots \ldots \ldots \ldots \ldots \ldots \ldots$

3.11 SimMechanics Validation using UMD Full Scale Test Rig data $\Omega=$ $18 \mathrm{rpm} \quad z / R=.2 \ldots \ldots \ldots \ldots \ldots \ldots \ldots$

4.1 SimMechanics Model of tip rotor configuration . . . . . . . . . . 63

4.2 SimMechanics Visualizations of tip rotor configuration . . . . . 64

4.3 SimMechanics Model of quadrotor configuration . . . . . . . . . . 65

4.4 SimMechanics Visualizations of quadrotor configuration . . . . . 66

4.5 Time Response for Base Da Vinci III configuration . . . . . . . 67

4.6 Time response for base quadrotor configuration . . . . . . . . 70

4.7 Torque applied to rotors on base configuration . . . . . . . . . . 71

4.8 Time response for the quadrotor with a uneven inertia $\ldots . . .72$

4.9 Time response for the quadrotor with a uneven mass _ . . . . 73

4.10 Time response for the quadrotor with a uneven geometric twist . 74

4.11 Time Response for the quadrotor with a initial angular displacement 75

4.12 Torque Applied to rotors . . . . . . . . . . . . . . . 76

4.13 Time Response for the quadrotor with a initial linear velocity . . 77

4.14 Eigenvalue Magnitude for change in Rotor Dihedral . . . . . . . 79

4.15 Eigenvalue Magnitude for changing carrier vertical location . . . . 80

A.1 Class Diagram . . . . . . . . . . . . . . . . . . . . 93

B.1 How to: . . . . . . . . . . . . . . . . . 97 
B.2 SimMechanics Model of CoAxial HPH configuration . . . . . . . . 100

B.3 Carrier Time Response . . . . . . . . . . . . . . . . . . . . . . . . 102

B.4 SimMechanics Model of Elastic Blade element . . . . . . . . . . . 104 


\section{Nomenclature}

$\begin{array}{ll}\text { Variables } & \\ A, B, C, D, E, F & \text { Inertia tensor components } \\ a_{0} & \text { Airfoil lift curve slope, } 1 / \mathrm{rad} \\ A R & \text { Aspect ratio, }- \\ \vec{a}_{p} & \text { Particle linear acceleration, } \mathrm{m} / \mathrm{sec}^{2} \\ c & \text { Airfoil chord length, } \mathrm{m} \\ C_{d} & \text { Local drag coefficient, }- \\ C_{P} & \text { Coefficient of power } \\ C_{T r e q} & \text { Thrust coefficient Required, }- \\ e & \text { Oswald efficiency, }- \\ e & \text { error } \\ F & \text { External force, } N \\ h & \text { angular momentum } \\ I_{\beta} & \text { Blade flap inertia, } N \mathrm{~m}^{2} \\ k & \text { Current Time step number } \\ K_{\beta} & \text { Equivalent center spring stiffness, } \mathrm{Nm} / \mathrm{rad}\end{array}$


$L, M, N$

$N$

$p$

$p, q, r$

$Q$

$\vec{r}_{p / b}$

$R$

$r$

$r$

$R_{m}$

$U$

$u$

$u, v, w$

$\mathbf{v}$

$x$

$X, Y, Z$

$x, y, z$

$y$

Z

$z$

\section{Greek Symbols}

Aerodynamic lift force function

Torque components, $\mathrm{Nm}$

Number of elements

Apparent order

Angular velocity components, $\mathrm{rad} / \mathrm{sec}$

External torque, $\mathrm{Nm}$

Distance between body origin and body particle, $m$

Rotor radius, $m$

Grid refinement factor

Non-dimensional radial coordinate, -

Rotation matrix

Local freestream velocity, $\mathrm{m} / \mathrm{sec}$

Input

Linear velocity components, $\mathrm{m} / \mathrm{sec}$

Body linear velocity, $\mathrm{m} / \mathrm{sec}$

State vector

Force components, $N$

Linear distance components, $m$

Output vector

Eigenvalues

Rotor vertical distance above the ground 


$\begin{array}{ll}\alpha & \text { Local airfoil angle of attack, } \mathrm{rad} \\ \beta & \text { Blade flap angle, } \mathrm{rad} \\ \dot{\beta} & \text { Blade flap angular velocity, } \mathrm{rad} / \mathrm{sec} \\ \lambda & \text { Inflow ratio, }- \\ \lambda_{\beta}^{2} & \text { Flap frequency ratio } \\ \omega & \text { Body angular velocity, } \mathrm{rad} / \mathrm{sec} \\ \mu & \text { Kinematic viscosity } \\ \Omega & \text { Rotor rotation speed }, \mathrm{rad} / \mathrm{sec} \\ \phi & \text { Inflow angle, } \mathrm{rad} \\ \psi & \text { Azimuth position, } \mathrm{rad} \\ \rho & \text { Air density, } \mathrm{kg} / \mathrm{m}^{3} \\ \sigma & \text { Rotor solidity, }- \\ \theta & \text { Geometric twist } / \mathrm{pitch}, \mathrm{rad}\end{array}$

Abbreviations

AHS

American Helicopter Society

BEMT

Combined Blade Element-Momentum Theory

DOF

Degrees of Freedom

FSTR Full Scale Test Rig

GCI Grid convergence index

GETR Ground Effect Test Rig

GUI Graphical User Interface

HPA Human Powered Aircraft 


$\begin{array}{ll}\text { HPH } & \text { Human Powered Helicopter } \\ \text { IGE } & \text { In Ground Effect } \\ \text { MBSSIM } & \text { Multi-Body System Simulation } \\ \text { OGE } & \text { Out of Ground Effect } \\ \text { PID } & \text { Proportional-Integral-Derivative (controller) } \\ \text { UMD } & \text { University of Maryland }\end{array}$

Subscripts

o Initial value 


\section{Chapter 1}

\section{Introduction}

\subsection{Competition}

In 1980, the American Helicopter Society (AHS) announced the Igor I. Sikorsky Human Powered Helicopter(HPH) Competition. The prize is a quarter million dollars for the first group to reach a height of 3 meters, stay aloft for sixty seconds and stay within a 10 meter square box[9]. This competition continues today.

Between 1980 and today, there has been a long list of attempts. Hawkins[5] presents a list of historical vehicles up to 1996. The Da Vinci III stands out on Hawkins' list because it was the first to achieve lift-off. The Da Vinci series was built by a team from California Polytechnic State University San Luis Obispo in 1989[10]. The Da Vinci III used propellers at the tips of a much larger main rotor with the rider slung under the vehicle along the axis of rotation of the main rotor[6]. One large main rotor allows this configuration to have low structural weight for the amount of thrust produced. Table 2.1 contains a more detailed description of the Da Vinci III. The record flight of the vehicle was plagued by stability issues shortly after lift-off, [11].

The Gamera from UMD is the most recent attempt and has also become the 
current record holder, setting the record for the longest and highest flight. The Gamera I successfully achieved flight in 2011[12], and the Gamera II continues to set records in Fall 2012[13]. The Gamera configuration consists of 4 direct-drive rotors[14], shown in figure 1.1.

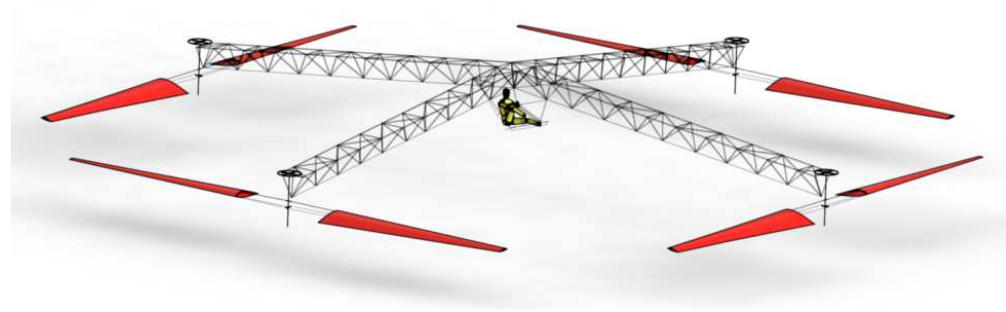

Figure 1.1: Gamera II Quadrotor configuration,[1]

The rider and the rotors are positioned near the ground with the structure extending upwards. This design was originally successful as Nihon University with the Yuri[15] shown in figure 1.2. The distribution of thrust among four rotors give the configuration better stability characteristics than the Da Vinci configuration.

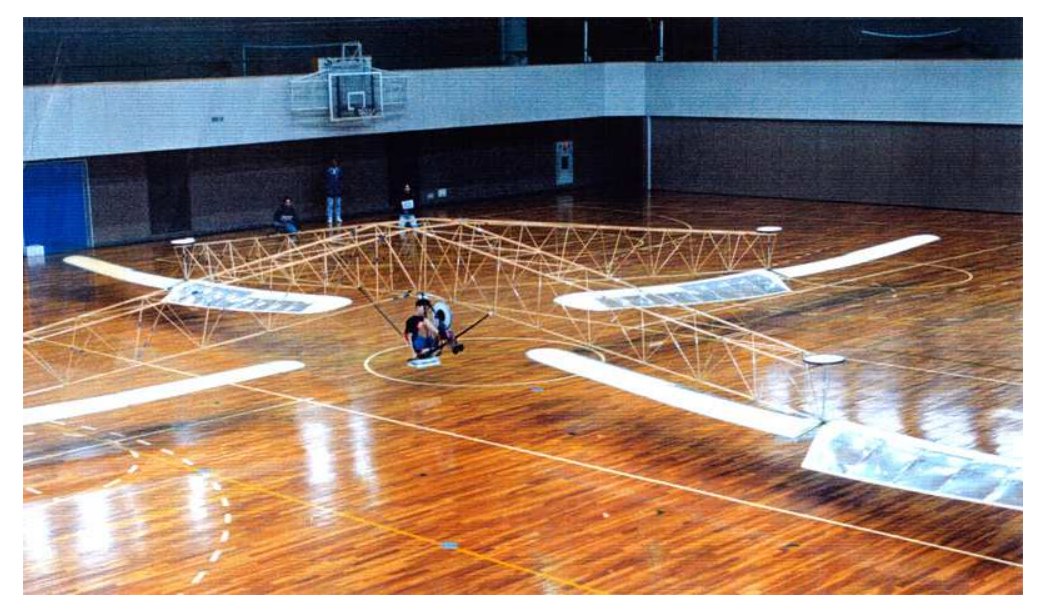

Figure 1.2: Yuri I quadrotor configuration,[2]

The Gamera II was able to climb to approximately 8 feet. Figure 1.3 shows the Gamera II in this 8 foot flight. Translation was a issue for the Yuri I and the same can be seen in the Gamera II,[16, 2]. Table 2.1 contains some of the important characteristics of the Gamera II compiled from works from UMD. 


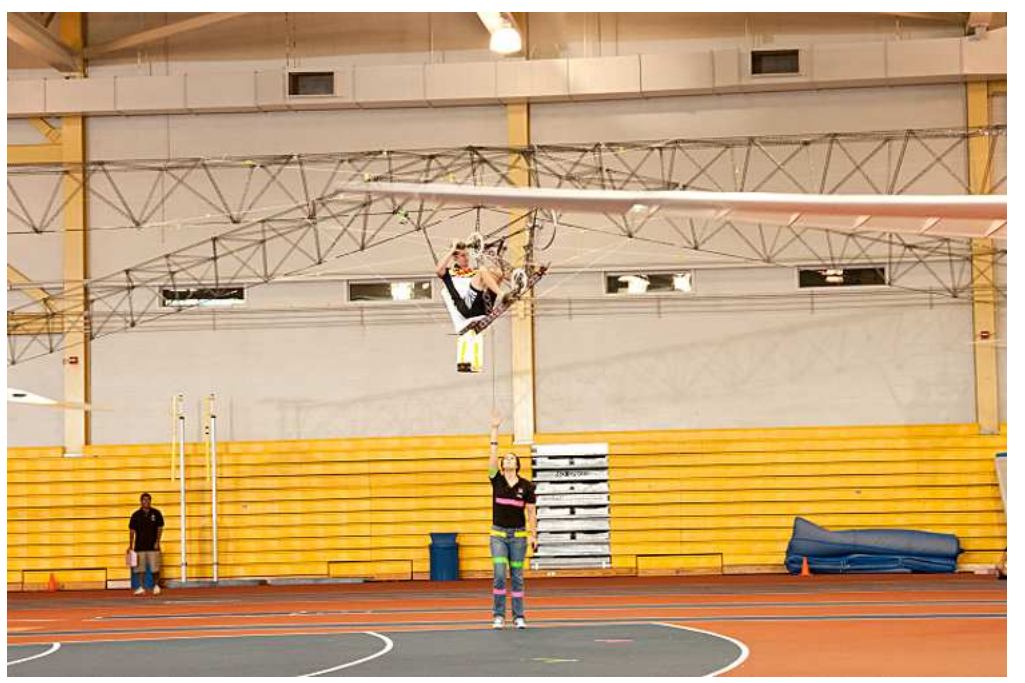

Figure 1.3: Gamera II's Highest flight,[3]

The Da Vinci Series from Cal Poly and the Gamera series from UMD demonstrate requirements of the problem. The $\mathrm{HPH}$ competition requires a vehicle that can meet aerodynamic, structural and dynamic stability requirements. The aerodynamic and structural requirements are coupled. The lifting surfaces of the vehicle must produce thrust greater than the weight of the vehicle, but at a power level that is achievable by a human powerplant. The human powerplant has limited torque, actuation speed and endurance. The structure must support the loads experienced by the HPH without failure, but cannot be heavier than the total thrust the vehicle can produce. Dynamic stability is an important requirement that is revealed from investigation of past attempts that have achieved lift-off. The Yuri and Gamera experienced uncontrolled lateral translation, while the Da Vinci experienced lateral axis angular oscillations. The HPH cannot sustain flight for 60 seconds or reach 3 meter of altitude without meeting requirements of stability. These three requirements must be satisfied to clinch the Sikorsky Prize. 


\subsection{Previous Work}

There has been many attempts at the prize. Each attempt requires thousands of man-hours of design and construction. This time holds no value for the current work on the HPH unless the analysis and results are recorded. The analysis focuses on the three requirements described above.

The aerodynamic requirements for the HPH are assessed through analysis of rotor performance. Gilad[17] validated theoretical aerodynamic models using the experiments conducted for the Gamera project. Gilad validated combined blade element-momentum theory, and prescribed wake theory with experimental data from a full scale Gamera blade test setup, and a sub-scale ground effect test setup. Gilad's report concluded that the combined blade element-momentum theory were sufficient for preliminary studies of flexible rotor behavior. Gilad also concluded that modelling blade bending was necessary to achieve accurate performance predictions. Gilad's thesis contributes to the HPH knowledge through validation of classical aerodynamic model. These aerodynamic model can now be used in the design process to determine if the aerodynamic requirement has been met.

Larwood and Saiki[10] treat the aerodynamic design and testing for the Da Vinci configuration. Larwood and Saiki used a free wake analysis code to determine performance trends for the main and tip rotors of the Da Vinci. The code had inputs for rotor geometry, thrust and rotational speed, and would return rotor profile and induced power requirements. Two Hundred cases were run on the NASA-Ames CRAY XM-P computer. The Da Vinci tip propellers were numerically optimized by finding the minimum induced loss propeller geometry. The propellers were tested in the 7-by-10 foot subsonic wind tunnel in the NASA Ames research center at Moffett Field. The results of the wind tunnel tests showed that the maximum propeller efficiency was well predicted by the theoretical analysis. The experimental tests, however, showed the propellers performed better at higher advance ratios, than was shown by the theoretical analysis. The aerodynamic numerical and experimental analysis data was applied to the design of the Da Vinci III. The Da Vinci III would go on to set the record for the first 
lift-off by a Human Powered Helicopter.

The aerodynamic requirements of the HPH have been investigated and the results used in the design of the respective HPH, as shown by these two reports.

The structural requirements for the HPH are assessed through analysis of the vehicle design loads and the subsequent design weight required to withstand those loads. Staruk et al. [12], Berry et al. [1] and ,Schmaus et al. [14] summarize the design and construction of the Gamera I \& Gamera II structure. The effort focuses on the weight of the vehicle and the effort involved to reduce it. Weight reduction must be achieved while still maintaining a safety margin. The reports focus on blade and airframe design. The blade had a mostly empty design using expanded polystyrene foam trailing edge ribs with a single layer of Mylar film used as the blade skin. The leading edge constructed of expanded polystyrene foam ribs and a extruded polystyrene foam shell. The blade spar was a triangular truss structure of pultruded uni-directional carbon fiber-vinyl ester composite tubes at the corners with a shear web of discrete unidirection carbon fiber-epoxy composite bundles laid at 45 degree angles span-wise. The triangular edge of the spar was set at the bottom of the airfoil section, with the triangle point at the top of the airfoil. Several experimental tests were performed which correlated well with Euler-Bernoulli theory and ANSYS numerical analysis. The Airframe structural analysis for the Gamera I used space truss theory and a genetic algorithm for minimization of weight. The airframe truss structure was a scaled version of the blade spar design. The Gamera II airframe design used Micro-Trusses structure with varied diameter. Figure 1.4 shows the empty triangular micro truss structure for the Gamera project. 


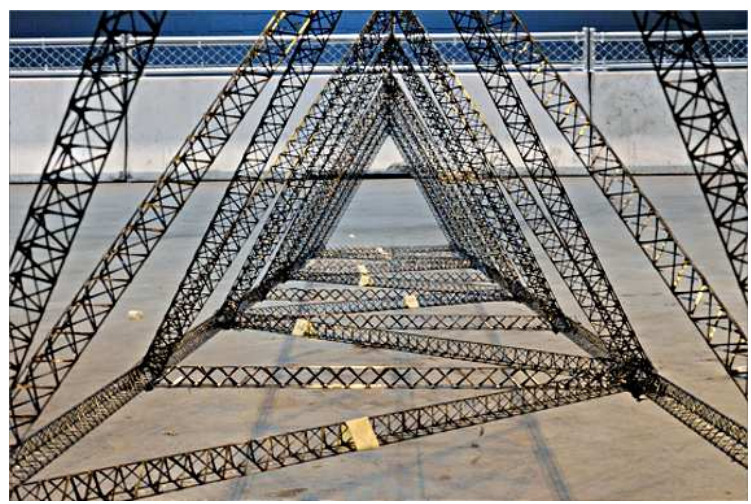

Figure 1.4: Gamera Triangular Truss Structure,[3]

Work done on human powered fixed wing aircraft(HPA) is applicable to the HPH. [18, 19, 20, 21, 22, 23, 24] detail structural analysis and testing related to the HPA project. Figure 1.5 shows an early Daedalus project design that has wing properties and construction similar to the HPH designs.

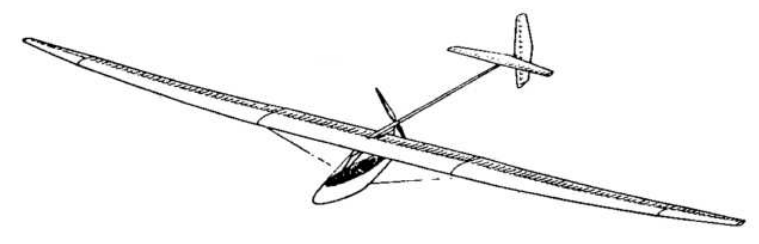

Figure 1.5: Phase I Preliminary Design of Daedalus Project, [4]

The first human powered ornithopter flew at the University of Toronto in 2010, and was accompanied by more modern report in the area of lightweight structures, and low speed aerodynamics $([25,26,27,28,29,30])$. Figure 1.6 shows the University of Toronto Snowbird, which has lightweight structures and large lifting surface spars similar to the HPH. This same team is now testing a human powered helicopter, named Atlas, and has already achieved first flight [31]. These reports contribute to the HPH knowledge through the development of light weight structures to meet the structural requirements for the HPH. 


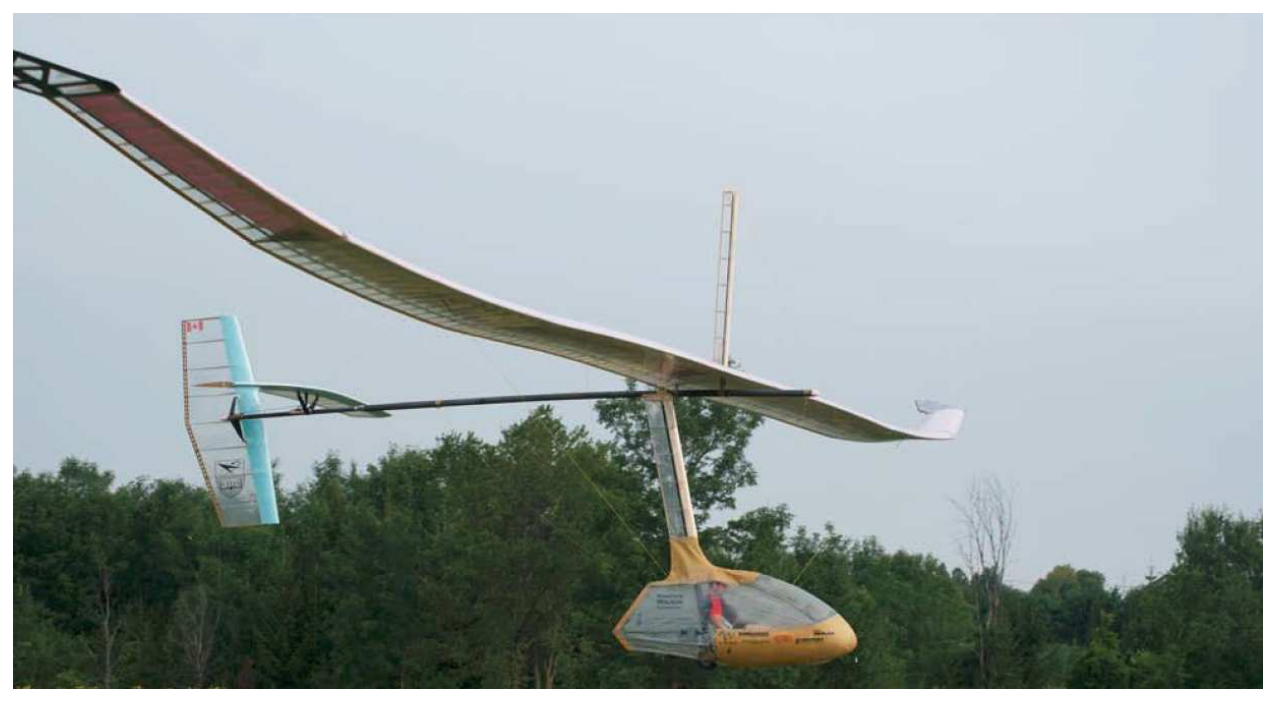

Figure 1.6: Human Powered Ornithopter, Snowbird,[4]

Hawkins[5] provided a wide breadth of HPH-specific preliminary design and performance analysis. Hawkins performs configuration trade studies using estimates for configuration weight, size and power required. Hawkins adds detail to the configuration trade study by including effects of airfoil selection, rotor radius, rotor tip speed, rotor chord, rotor twist and rotor taper. Hawkins' performance analysis culminated in a 1-dof detailed dynamics model to assess the HPH flight profile. This model included ground effects, blade coning, vertical climb/descent inflow, blade pitch control and a engine model. Hawkins' simulated the VK-1 configuration shown in figure 1.7. The simulation showed that this configuration was a "strong candidate" for the competition. Hawkins' concluded that "brute force" in any one area will not be sufficient to claim this prize. Perhaps the most important contribution that Hawkins' had is a discussion on stability and control analysis:

"It can not be overemphasized how important a complete stability analysis of the HPH could be to the overall success of the project. Understanding and leveraging the system dynamics is one of the key components to success." Hawkins [5] 

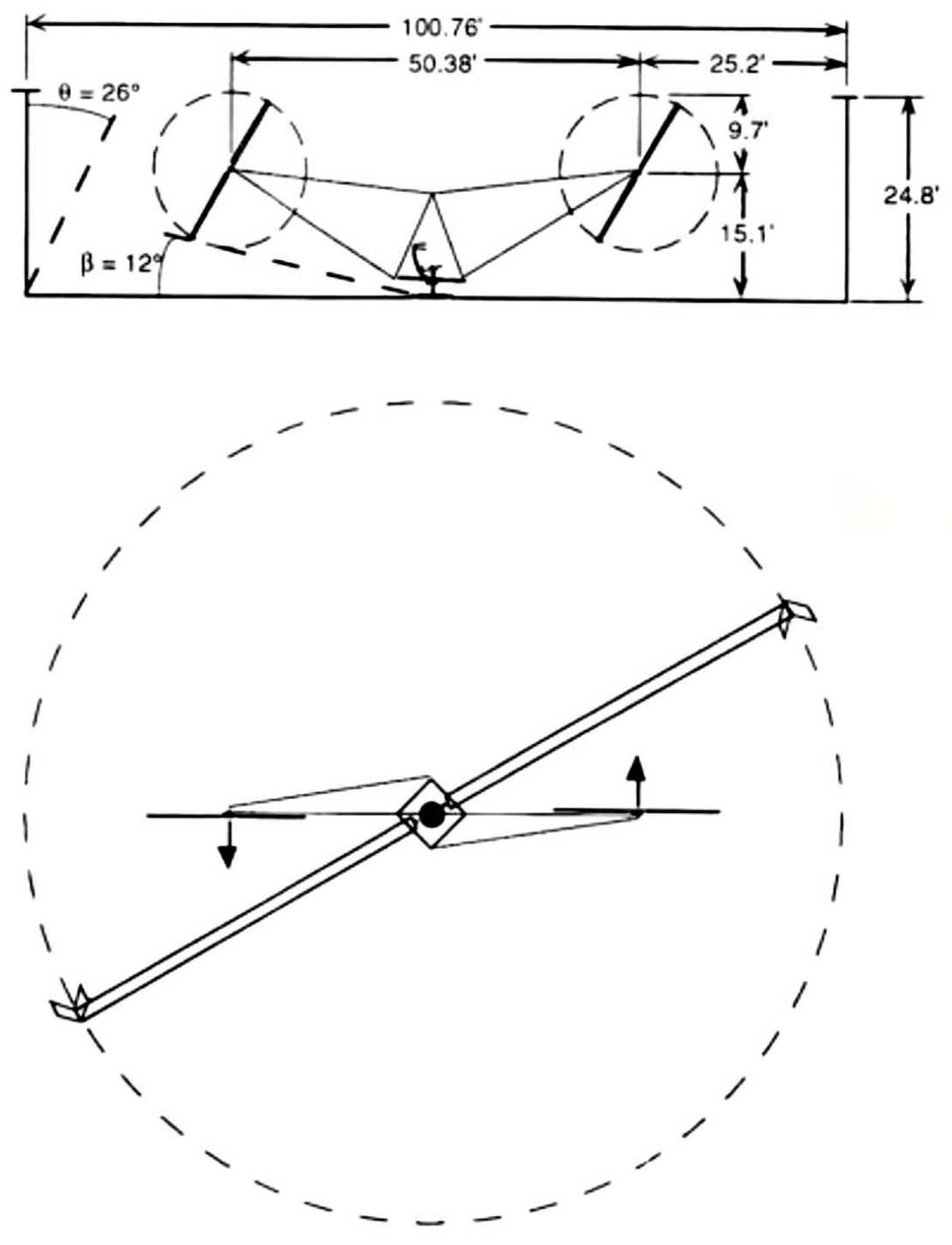

Figure 1.7: The VK-1 single main rotor with twin tails \& winglets [5] 
Stability is a requirement of the HPH. Totah, Patterson[6] and Guttieri [32] focused on the stability and possible control of the Da Vinci series from Cal Poly. Totah and Patterson used a five degree of freedom kinematic model to study the affect of control on the Da Vinci II. The dynamic model used equations and assumptions presented by Bramwell[7]. The dynamic model ignored stall effect, pitch-flap coupling, and vertical, longitudinal and lateral axis coupling. The dynamic model made the quasi-steady assumption and small angle assumption. The dynamic model included an aeroelastic effect through assumption of a constant flap or coning angle. The dynamic model included ground effect and tip-losses. Totah and Patterson solved the equations of motion through the Adams-Bashforth numerical integrator.

The dynamic response shows that the Da Vinci configuration without a control system is unstable. This conclusion agrees with video footage of the Da Vinci III[11]. Totah and Patterson modelled a tip mounted control system, that deflects a blade section at each tip. The control system uses a linear control law based on the differential tip height-to-ground sensed optically at each tip. Figure 1.8 show the tip-mounted control system. As an aside, the tip mounted control system was recently implemented by NTS works Upturn, [33].

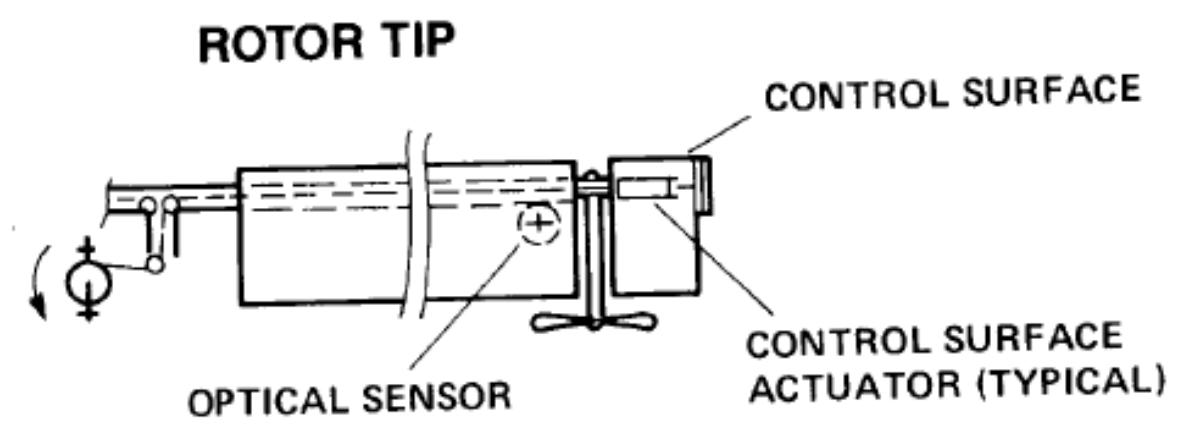

Figure 1.8: Tip-Mounted Control system for Da Vinci II[6]

Figure 1.9 shows the full Totah and Patterson model. Totah and Patterson present response data for the control simulation showing a stable system. The Da Vinci II model with a control system with rate and position limiting concluded to be stable by Totah and Patterson. Totah and Patterson concluded the dynamic 
model could not be validated due to the lack of available flight test data, and was therefore considered preliminary.

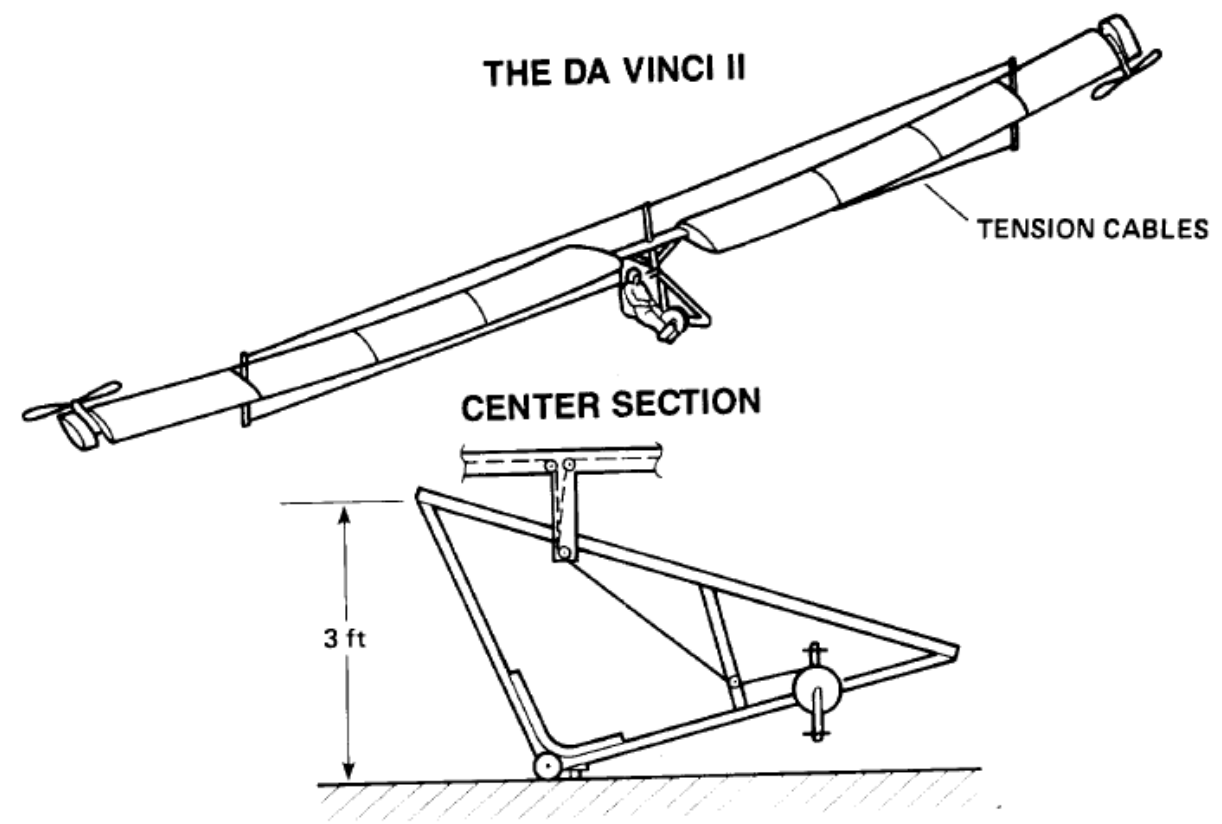

Figure 1.9: Da Vinci configuration[6]

Guttieri's report on the HPH stability and control was written after the Totah and Patterson report. Guttieri derives the dynamics equations of the Da Vinci from first principles and assesses the quasi-steady assumption for validity by comparing the blade response when the quasi-steady assumption is used to the response when it is not used. Guttieri concludes the quasi-steady assumption for analysis of the dynamic response of the $\mathrm{HPH}$ is inappropriate. The conclusion of Guttieri nullifies the conclusions of Totah and Patterson. There have been no contributions to knowledge of the stability requirement for the human powered helicopter problem.

The HPH problem has aerodynamics, structures and dynamic stability requirements. Aerodynamics and structures have been treated in numerous reports. The stability requirement of the HPH has not been treated, and cannot be easily approached by traditional helicopter dynamics methods. 


\subsection{Solution}

Traditional helicopter dynamic analysis builds the equations of motion from the Newton-Euler equations. These non-linear equations are linearized for preliminary analysis using several helicopter-specific assumptions([7, 34]). Traditional linear stability theory is used to determine the stability of the linearized equations. The assumptions, however, must be applied with discretion. The helicopter assumptions must be reassessed for validity if the configuration is altered or if the vehicle operates in a different flight regime.

Multi-body system simulation(MBSSIM) is an alternate approach to determining the stability characteristics of helicopter configurations. Multi-body system simulation is a discipline within Mechanical Engineering developed in the 1960s for treatment of complex satellite systems[35]. By the 1980s, computer software was developed to allow for simulation and animation of these complex systems. Systems are broken into discrete rigid bodies with non-zero mass and inertia. These bodies are then connected using joints to allow relative movement[36]. Forces and Moments are generated to set the system in motion through the use of compliant elements. Research has been done using the multi-body systems approach in the field of Aeronautic and Machinery Design[37, 38], Biomechanics[39], and Robotics[40]. Flexibility and reconfigurability are the major advantages of MBSSIM. MBSSIM allow assumptions to be implemented by the designer, through the development of discrete physics models. MBSSIM relies on numerical integration, thus the stability of the system cannot be determined directly from the system.

MBSSIM will produce the dynamic response of the system. System Identification is used to find a linear state space system from the dynamic response. System identification is a field of control engineering whereby statistical methods are used to determine parameters of a mathematical model from a set of measured data [41]. System identification can be used in any field where the researcher requires a mathematical model to be produced from a data set. Specific applications are found in aerospace and aeronautic [42]. System identification 
will produce a discrete linear state space system. The stability properties of the discrete linear state space system can be found through common linear stability analysis theory.

This method solves the problem of determining the stability characteristics of a human-powered helicopter, without the use of traditional helicopter dynamic analysis. Figure 1.10 shows a information flow chart of the method developed and implemented in this thesis. 


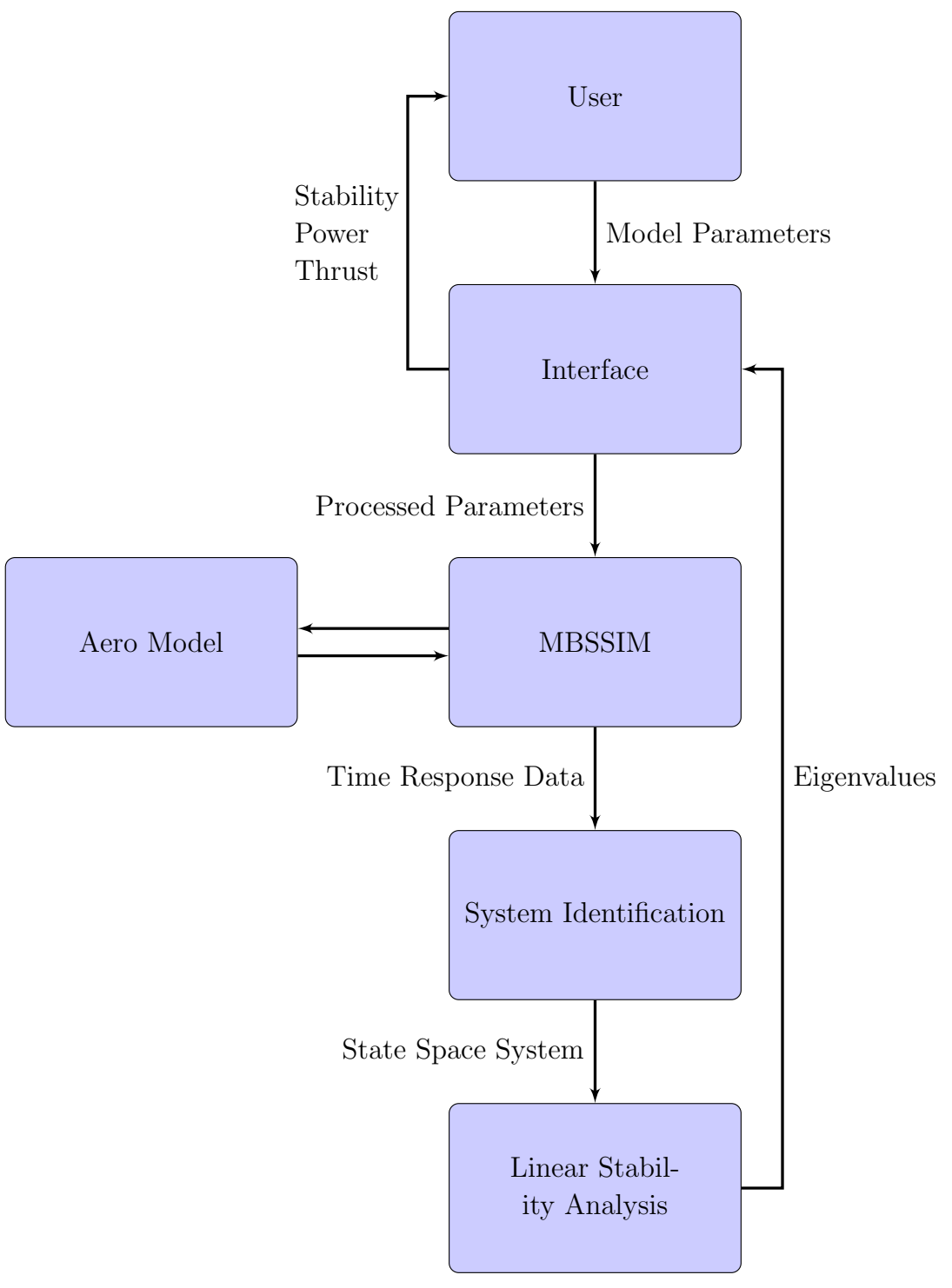

Figure 1.10: Multi-body Simulation Method 


\subsection{Thesis Scope}

This thesis contribute to the knowledge of HPH stability through the development of a method, which uses of multi-body system simulation, system identification and linear stability analysis theory.

This thesis will present traditional helicopter dynamic theory. Presentation of the traditional helicopter dynamic theory will be used to justify its abandonment. This justification will focus on comparison of traditional helicopters and HPH using non-dimensional terms important in rotor blade flap response. Helicopter blade flap dynamics will be derived.

Combined Blade Element-Momentum theory is presented for use in modelling the aerodynamics of the HPH. Combined Blade Element-Momentum theory discretizes a rotor blade into blade elements. The force acting at each blade element will be a function of the local conditions. Momentum theory produces an equation for inflow which models the effects of the increase freestream velocity through the rotor due to thrust.

This thesis will briefly discuss MBSSIM, system identification, and linear stability analysis theory. MBSSIM uses first principle equations to automatically generate equations of motion based on the discrete bodies and joints present in the system. System identification finds parameters that will give a response that matches the data set best, using probability techniques along with the equation for the desired mathematical model. In this these the desired mathematical model will be a discrete-time linear state space system of varying order. The system identification section will treat system identification as a black box. The section will only discuss the inputs and outputs to system identification and the options available to the user. The solution to and stability of the discrete-time linear state space equations will be discussed in the linear stability analysis section. The stability analysis will present several theorem for determining the stability of a linear system, but will not present proofs for them.

This thesis will discuss implementation of the HPH system into a commercial MBSSIM Graphical User Interface(GUI), and the interfacing program used to 
manipulate the GUI. MathWorks ${ }^{\circledR}$ SimMechanics toolbox ${ }^{\mathrm{TM}}$ is the MBSSIM used in this thesis.

The interface to the MBSSIM GUI is developed in MathWorks ${ }^{\circledR}$ MATLAB ${ }^{\circledR}$ for this thesis to allow for scripting of interactions with the MBSSIM. The interface uses object-oriented programming techniques to control data access and flow with the MBSSIM. The aerodynamic model is verified through a Richardson extrapolation and an effort is made to validate it with experimental data from UMD's Gamera project. The experimental data used didn't include experimental uncertainty data, and thus the aerodynamic model will not be validated due to insufficiencies in experimental data.

The results of this thesis present a demonstration of the method for dynamic analysis of the Gamera quadrotor configuration. Time responses for the base quadrotor configuration and the base quadrotor configuration with varied properties are presented. The stability properties identified through system identification and linear stability analysis are presented. Parametric studies are presented to demonstrate the methods use in a design capacity. The results show this method of dynamics analysis can be used to determine the dynamic response and stability properties of a HPH configuration. This work is only meant to discuss tool development; and does not endorse any HPH design decisions.

\subsection{Thesis Outline}

Section 2.2 discusses traditional helicopter vehicle dynamics. This section will also present a formal argument against the use of traditional helicopter dynamic analysis. MBSSIM is be discussed in section 2.3. Rotor aerodynamic theory will be presented in section 2.4. Next, the methods for system identification and stability analysis will be discussed in sections 2.5 and 2.6, respectively. In chapter 3, the specific implementation of MBSSIM will be discussed, along with the verification and validation of the implemented aerodynamic model. Finally, in Chapter 4, the author will demonstrate the abilities of this method. Section 
2.1 will discuss the traditional helicopter and a comparison to the HPH. 


\section{Chapter 2}

\section{Theory}

Helicopters produce required lift through the rotation of a lifting surface about a locally vertical axis. Control of a helicopter is found through the cyclic or collective change in the lifting surface pitch. The power required for lifting surface rotation is produced from an on-board heat engine. The difference between HPHs and traditional helicopters is the heat engine. HPHs have limited power and endurance, compared to the traditional helicopter. This difference leads to a branching of the design characteristics.

\subsection{Helicopter Configurations}

Traditional helicopter heat engines have a high power capability and enable the production of a large amount of thrust via helicopter blades. The high power capability creates a large design space for the traditional helicopter, with design missions spanning cargo transport to high speed reconnaissance[43]. This large design space allows the traditional helicopter to be designed to meet structure, aerodynamic and dynamic stability requirements with a margin in each. Characteristics for some traditional helicopters from, Padfield[34], are shown in Table 2.1. Figure 2.1 shows a traditional helicopter. 


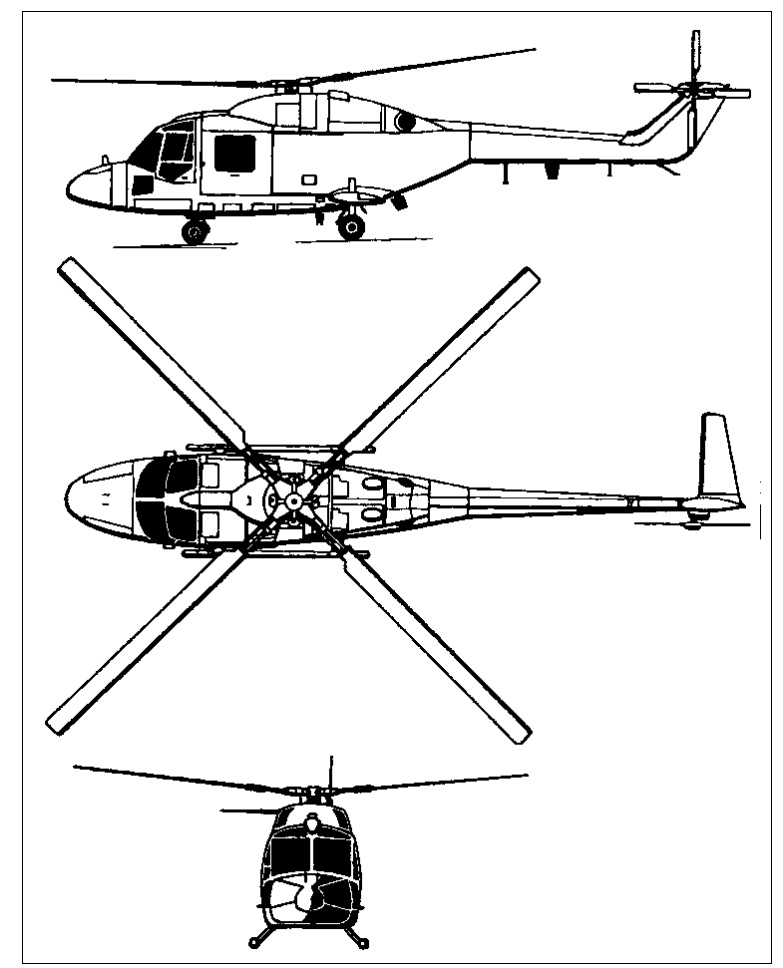

Figure 2.1: Lynx DRA Research Helicopter

\subsubsection{The Human-Powered Helicopter Configuration}

The power specification for a human, approximately $1 \mathrm{~kW}$, confines the design space for a human-powered helicopter. Designers are driven to selecting configurations that share the qualities of being light-weight, 55-100 lbs, and high total wing spans, between 100-130 ft[44, 5]. For these reasons, the most successful materials for HPH are carbon fiber composite, balsa wood, stiff foam, and some type of shrink wrap film. Rotor blades are mostly empty with a carbon fiber composite spar, wood or foam ribs and a shrink wrapped skin wing skin to make and keep a smooth airfoil shape.

The four most common HPH designs are the coaxial rotor, tip propellerdriven main rotor, a quadrotor, and the conventional main and tail rotor[5]. The quadrotor and the tip propeller-driven main rotor configurations will be examined in this thesis due to their success. Characteristics for the Da Vinci III and Gamera II, will utilized for the Quadrotor and tip-propeller driven main 
rotor, respectively.

Table 2.1: Helicopter Characteristics

\begin{tabular}{|l|l|l|l|l|l|}
\hline Characteristic & Lynx & Bo105 & Puma & Gamera I & Da Vinci III \\
\hline Rotor Radius, $R(\mathrm{~m})$ & 6.4 & 4.91 & 7.5 & 6.5 & 20.42 \\
\hline Rotation Speed, $\Omega(\mathrm{rad} / \mathrm{s})$ & 35.63 & 44.4 & 27 & 1.8 & .6283 \\
\hline Blades \# (-) & 4 & 4 & 4 & 2 & 2 \\
\hline$K_{\beta}(\mathrm{Nm} / \mathrm{rad})$ & 166,352 & 113,330 & 48,149 & - & 74,167 \\
\hline Lock Number, $\gamma(-)$ & 7.12 & 5.087 & 9.374 & - & 399.07 \\
\hline Flap Frequency Ratio, $\lambda_{\beta}^{2}$ & 1.193 & 1.248 & 1.052 & - & 20.77 \\
\hline Power Loading $(\mathrm{kg} / \mathrm{kW})$ & 3.1916 & 3.9936 & 2.47 & 160 & 30.19 \\
\hline Disk Loading $\left(\mathrm{kg} / \mathrm{m}^{2}\right)$ & 105.3 & 91.25 & 103.2 & 1.2 & .098 \\
\hline Tip Reynold's \# $\left(\times 10^{5}\right)$ & 58.5 & 38.6 & 71.7 & $5-9$ & 6 \\
\hline
\end{tabular}




\subsection{Helicopter Dynamics}

We begin our discussion of helicopter dynamics with a quote to describe the complexity taken on in helicopter dynamics analysis.

"...Not only is the aerodynamic environment of the rotor unsteady, but at the same time it is also non-linear, governed by noninfinitesimal motion and periodicity in conditions traditionally held constant with fixed wing applications...In addition to the problems directly arising from unsteady aerodynamics those related to the fact that the rotor blades of contemporary helicopter rotors are 'structural lightweights'compared with their fixed wing counterparts. They achieve a large measure of their stiffening from the tension induced by the rotational centrifugal force field. The rotational environment of the rotor blades also give rise to a host of rotation-related phenomena: gyroscopic characteristics, Coriolis forces, and a variety of non-linear inertial loadings. While still governed principally by linear operators, the resulting aeroelastic description of rotor blade elastic responses is consequently fraught with non-linearities that modify the result obtained using only linear analysis." - Bielawa [45]

The main rotor will be the centerpiece of this discussion of helicopter dynamics. Helicopter main rotor blades are designed to be flexible, in contrast to fixed wing aircraft which are generally designed to be static. The degrees of freedom for the blades of the main rotor are separated into out-of-plane rotation, in-plane rotation about the vertical axis, and in-plane rotation about the span-wise axis, which are known as flap, lag and feather, respectively. Figure 2.2 shows the rotor degrees of freedom for a hinged rotor. 


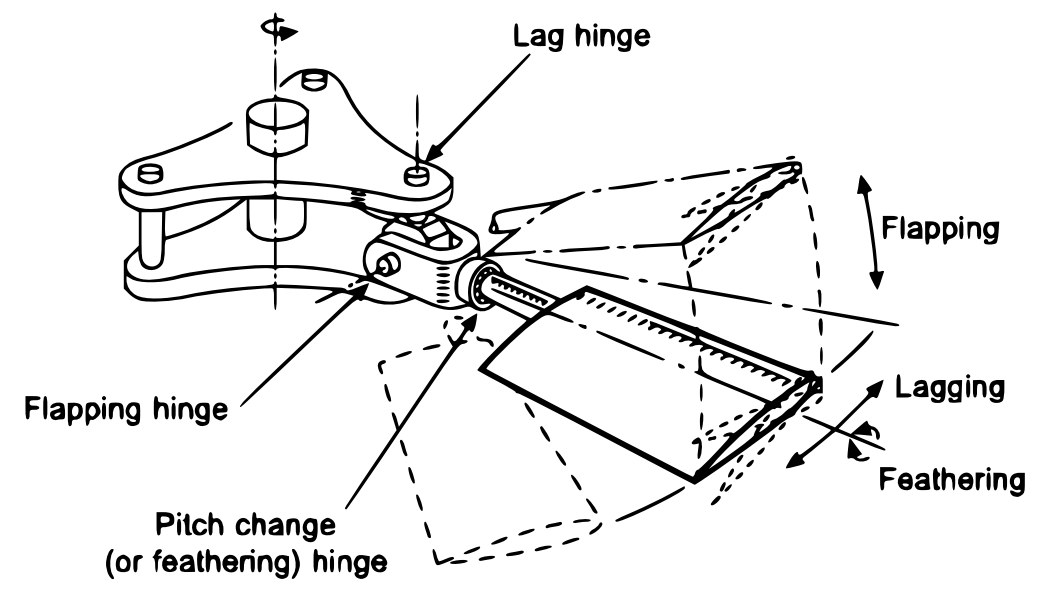

Figure 2.2: Hinged main rotor degrees of freedom, [7]

Traditional helicopters use the swash plate mechanism, shown in figure 2.3 to change the feather degree of freedom in a collective or cyclic pattern, this is the main control mechanism for a traditional helicopter

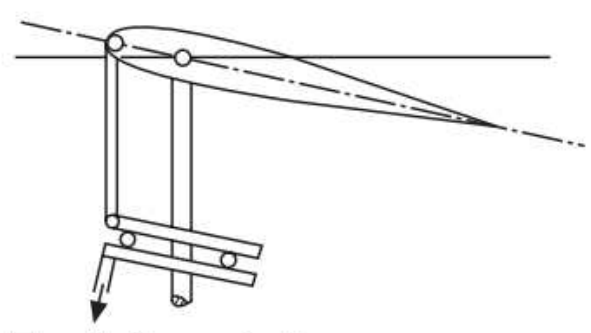

To pilot's control

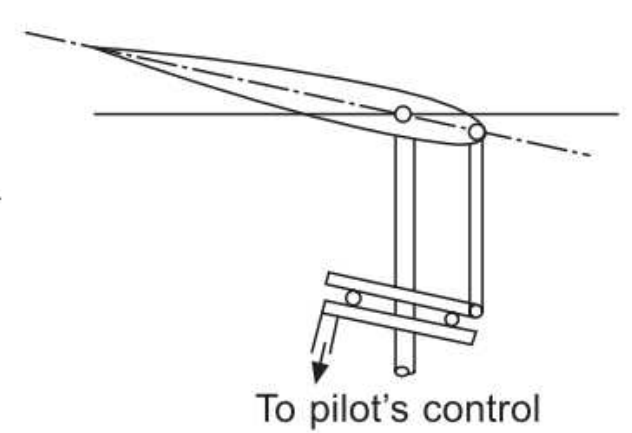

To pilot's control

Figure 2.3: Helicopter Swashplate mechanism, [7]

A hingeless rotor is typical for most HPH and the degrees of freedom are described the same but for the addition of elasticity in the blade response. Flap is the focus of helicopter dynamics literature([7],[34]) in preliminary analysis phase.

Reassessment of helicopter dynamic analysis theory is required due to the differences between a HPH and the traditional helicopter. Dynamic analysis for an air vehicle involves the quantification and combination of the aerodynamic, structural and inertial responses. Furthermore, an individual set of equations must be found for each helicopter configuration evaluated. The simplifying assump- 
tions cannot be used in dynamic analysis if the components assumed negligible are not. Each assumption must be evaluated to determine the difference in responses. The assumption can be used if the difference is negligible. Bramwell[7] and Padfield[34] are referenced for all the material in this section.

\subsubsection{Rigid Body Motion}

This section will derive the equations for general translation and rotation of a rigid body as a preface to the discussion of blade flap dynamics. Beginning with the fundamental Newton equation,

$$
\begin{array}{r}
F=m \frac{d \mathbf{v}}{d t} \\
Q=\frac{d \mathbf{h}}{d t}=I \frac{d \mathbf{\Omega}}{d t}
\end{array}
$$

, where $Q$ is torque and $F$ is force. The presence of rotational velocity requires the use of

$$
F=m\left(\frac{d \mathbf{v}}{d t}+\Omega \times \mathbf{v}\right)
$$

, where $\Omega$ and $\mathbf{v}$ are the angular and linear velocities vectors respectively.

The components of force and torque acting on the body are

$$
\begin{aligned}
& \vec{F}=X \hat{i}+Y \hat{j}+Z \hat{k} \\
& \vec{Q}=L \hat{i}+M \hat{j}+N \hat{k}
\end{aligned}
$$

The acceleration is expanded as, 


$$
\begin{aligned}
& a_{x}=\dot{u}-r v+q w-x\left(q^{2}+r^{2}\right)+y(p q-\dot{r})+z(p r+\dot{q}) \\
& a_{y}=\dot{v}-p w+r u-y\left(p^{2}+r^{2}\right)+z(q r-\dot{p})+x(p q+\dot{r}) \\
& a_{z}=\dot{w}-q u-p v-z\left(p^{2}+r^{2}\right)+x(p r-\dot{q})+y(q r+\dot{p})
\end{aligned}
$$

,where

$$
\begin{array}{r}
\vec{a}_{b}=a_{x} \hat{i}+a_{y} \hat{j}+a_{z} \hat{k} \\
\vec{\omega}_{b}=p \hat{i}+q \hat{j}+r \hat{k} \\
\vec{r}_{p / b}=x \hat{i}+y \hat{j}+z \hat{k}
\end{array}
$$

The total force is found by integrating the force for every point on the body. Assuming a body fixed coordinate system, B, at the center of mass, the differential equations for linear motion of a rigid body is given as

$$
\begin{aligned}
& X=M_{b}(\dot{u}-r v+q w) \\
& Y=M_{b}(\dot{v}-p w+r u) \\
& Z=M_{b}(\dot{w}-q u+p v)
\end{aligned}
$$

,where

$$
\mathbf{v}=u \hat{i}+v \hat{j}+w \hat{k}
$$

The derivation of the differential equations for rigid body rotational motion can be found in [7]. The Euler equations, given off-diagonal terms present in the inertia tensor, are 


$$
\begin{aligned}
& A \dot{p}-(B-C) q r+D\left(r^{2}-q^{2}\right)-E(p q+\dot{r})+F(p r-\dot{q})=L \\
& B \dot{q}-(C-A) r p+E\left(p^{2}-r^{2}\right)-F(q r+\dot{p})+D(q p-\dot{r})=M \\
& C \dot{r}-(A-B) p q+F\left(q^{2}-p^{2}\right)-D(r p+\dot{q})+E(r q-\dot{p})=N
\end{aligned}
$$

$A, B, C, D, E, F$ are components of the inertia tensor, and are used by

$$
\left[\begin{array}{l}
h_{1} \\
h_{2} \\
h_{3}
\end{array}\right]=\left[\begin{array}{ccc}
A & -F & -E \\
-F & B & -D \\
-E & -D & C
\end{array}\right]\left[\begin{array}{l}
\omega_{1} \\
\omega_{2} \\
\omega_{3}
\end{array}\right]
$$

,where

$$
\begin{array}{ccc}
A=\sum m\left(y^{2}+z^{2}\right) & B=\sum m\left(x^{2}+z^{2}\right) & C=\sum m\left(x^{2}+y^{2}\right) \\
D=\sum m y z & E=\sum m x z & F=\sum m x y
\end{array}
$$

The differential equations given in 2.13 through 2.15 and 2.17 through 2.19, are the Newton-Euler equations and constitute a full set of differential equations for rigid body motion. The rotational equations in Eq. 2.17 through Eq. 2.19 are used to present the flap degree of freedom.

\subsubsection{Blade Flap}

Analysis of Blade Flap begins by summing the moments due to inertial motion and structural stiffness about the flap hinge to produce,

$$
K_{\beta} \beta=-\int_{0}^{R} r m(r)\left(r \ddot{\beta}+r \Omega^{2} \beta\right) d r
$$


The term $K_{\beta}$ is a measure of the blade hinge stiffness in the flap dof. The inertial terms $\ddot{\beta}$ and $\beta$ on the right hand side, represent the force due to outof-plane flapping acceleration, and in-plane centrifugal acceleration, respectively. This analysis make the assumption that there is no energy dissipation in the structural response; in subsequent analyses this would be important to model, but our discussion will diverge before this point.

The temporal differential equation is transitioned to a spatial differential equation by

$$
\begin{array}{r}
\psi=\Omega t \\
\frac{d^{2}}{d t^{2}}=\Omega^{2} \frac{d^{2}}{d \psi^{2}}
\end{array}
$$

The spatial differential equation is now

$$
\beta^{\prime \prime}+\lambda_{\beta}^{2} \beta=0
$$

, where $\lambda_{\beta}^{2}$ is called the flap frequency ratio and is defined as,

$$
\lambda_{\beta}^{2}=1+\frac{K_{\beta}}{I_{\beta} \Omega^{2}}
$$

The solution to the second order homogeneous differential equation 2.25 is

$$
\beta=c_{1} \cos \left(\lambda_{\beta} \psi\right)+c_{2} \sin \left(\lambda_{\beta} \psi\right)
$$

,where flap frequency ratio, $\lambda_{\beta}^{2}$, will define the undamped frequency of blade flap.

The moments present at the flapping hinge must be reassessed when fuselage pitch and roll velocity are added, because of the added angular velocity compo- 
nents. The angular velocity components in the $\hat{i}, \hat{j}, \hat{k}$ coordinate system shown in figure 2.4 .

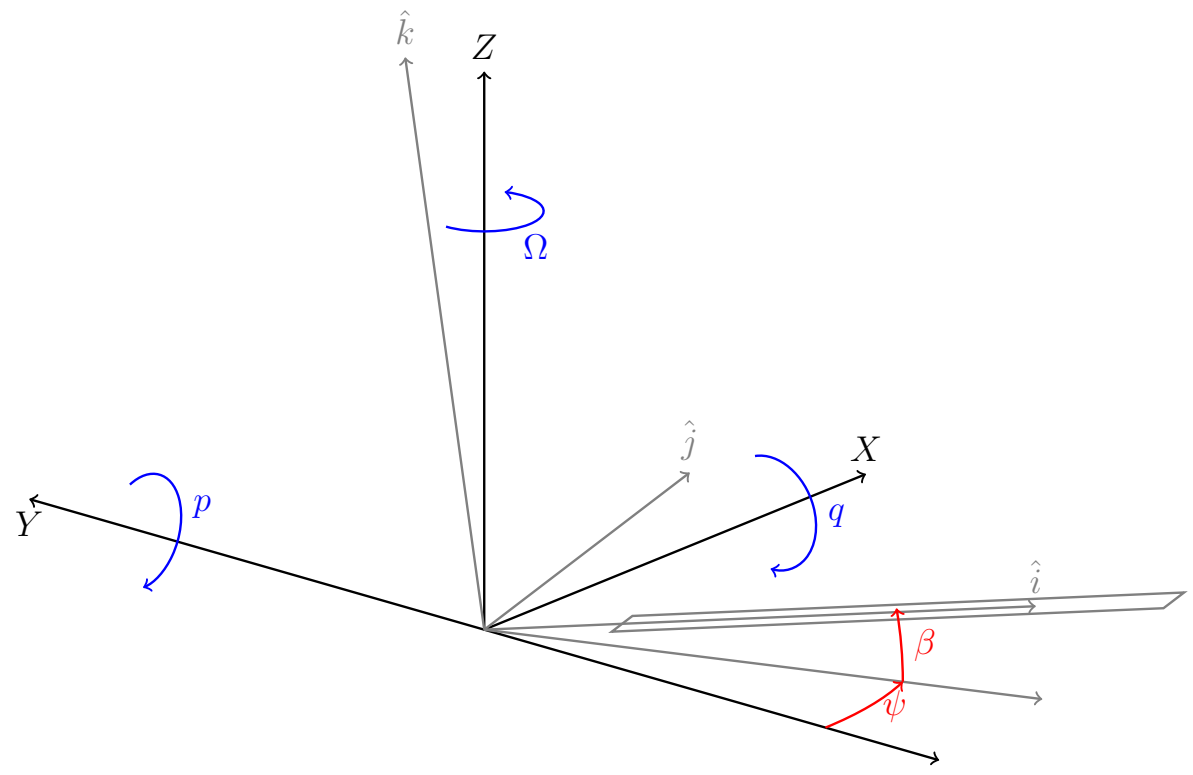

Figure 2.4: Flapping hinge coordinate system definition

For flapping and main rotor rotation only, angular velocity components of the blade are

$$
\omega=[\Omega \sin (\beta), \quad-\dot{\beta}, \quad \Omega \cos (\beta)]
$$

Matrix equation form is used when generating the equations that account for pitch and roll velocities; Angular components in matrix form are

$$
\omega=R_{m}(\beta)\left(R_{m}(\psi)\left[\begin{array}{c}
-p \\
q \\
0
\end{array}\right]+\left[\begin{array}{l}
0 \\
0 \\
\Omega
\end{array}\right]\right)+\left[\begin{array}{c}
0 \\
-\dot{\beta} \\
0
\end{array}\right]
$$

, where $R_{m}(\psi)$ and $R_{m}(\beta)$ are rotation matrices about z-axis and the y-axis, respectively. 
The component accelerations are derived relative to the origin of the flapping hinge. Euler equations are employed to produce blade flap dynamics. The simplification by Padfield from the full differential is

$$
\beta^{\prime \prime}+\lambda_{\beta}^{2} \beta=\frac{2}{\Omega}(p \cos (\psi)-q \sin (\psi))
$$

In this equation it is assumed that rotor rotation speed is much greater than the pitch and roll velocities; this assumption is usually valid for traditional helicopters.

Aerodynamic forces are added to the blade before solving the differential equation. Effects of drag are negated and a two-dimensional lift model is used for this analysis. The final flapping differential equation is

$$
\beta^{\prime \prime}+\lambda_{\beta}^{2} \beta=\frac{2}{\Omega}(p \cos (\psi)-q \sin (\psi))+\frac{1}{I_{\beta} \Omega^{2}} \int_{0}^{R} l(r, \psi) r d r
$$

, where

$$
l(r, \psi)=\frac{1}{2} \rho V^{2} c a_{0} \alpha
$$

$\theta, \alpha$, and $\phi$ are the geometric twist angle of attack and inflow angle, respectively. The quantities, $c$ and $a_{0}$ are the chord length and the lift curve slope, respectively. Figure 2.5 defines components of two-dimensional airfoil analysis. 


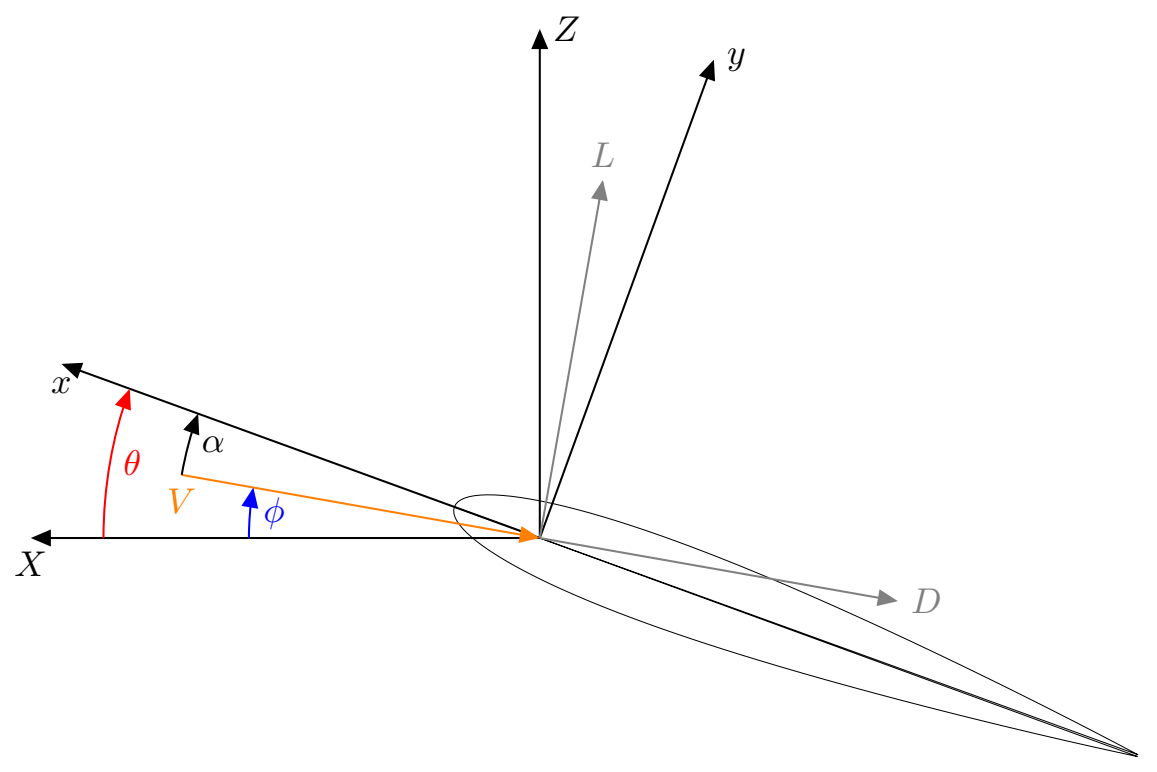

Figure 2.5: Two Dimensional Aerodynamic Model

After simplification, the final flapping differential equation is

$$
\beta^{\prime \prime}+\lambda_{\beta}^{2} \beta=\frac{2}{\Omega}(p \cos (\psi)-q \sin (\psi))+\frac{\gamma}{8}\left(\theta-\frac{4}{3} \lambda_{i}+\frac{p}{\Omega} \sin (\psi) \frac{q}{\Omega} \cos (\psi)\right)
$$

,where $\lambda_{i}$ and $\gamma$ are the inflow ratio and Lock number, respectively. The lock number is defined as

$$
\gamma=\frac{\rho c a_{0} R^{4}}{I_{\beta}}
$$

$\lambda_{i}$ and $\theta$ are the inflow ratio and blade pitch respectively; blade pitch is assumed to be a sinusoidal function of $\psi$ and is traditionally based on pilot control. The differential equation is solved using quasi-steady assumption.

The goal of this discussion is to check if the quasi-steady assumption is valid for the HPH configuration. This assumption may be used if the rotor reaction 
to a single control input is an order of magnitude faster than the change in the fuselage angular velocity or pilot control; it will allow the decoupling of the main rotor and fuselage dynamics.

The Lock number and Flap Frequency Ratio, $\gamma$ and $\lambda_{\beta}^{2}$, are used to determine if the quasi-steady assumption can be applied. These numbers represent the ratio of aerodynamic to inertial forces, and the ratio of structural to inertial forces, respectively. Table 2.1 shows the Lock number and flap frequency ratio for three traditional helicopters and the Da Vinci HPH; aerodynamic forces are greater than the inertial forces for all the helicopters presented. Notice that the HPH Lock number is 57 times greater than the average Lock number for the traditional helicopter. The flap frequency ratio is 18 times greater than the average flap frequency ratio for the traditional helicopter. The previous two facts imply that the aerodynamic and structural dynamic responses is much greater than the inertial response for the Da Vinci HPH.

Assessment of the goals for past HPH project teams makes this clear conceptually. The goal for past HPH projects is to build the vehicle bigger, lighter and stronger. The strength in the rotor blade spars and other structure will increase the flap frequency ratio. The Lock number will be increase as the rotor blades become bigger without a equivalent increase in blade weight.

Guttieri[32] concludes the response time for a disturbed Da Vinci rotor blade in flap is greater than 3 seconds. The change in fuselage angular velocity is not an order of magnitude slower than the change in rotor flap angular velocity, and thus the quasi-steady assumption cannot be used.

Totah[6] found, through simulation, the maximum pitch velocity is approximately $0.45 \mathrm{rad} / \mathrm{sec}$. The design rotation speed of the Da Vinci main rotor is approximately $0.63 \mathrm{rad} / \mathrm{sec}$. The rotor rotation speed was assumed to be an order of magnitude faster than the pitch velocity of the fuselage. This is not the case for HPH configurations, thus the assumption is not valid for the HPH.

The quasi-steady assumption and the rotation speed assumption will not be valid for the HPH. The equations will have to be re-derived by including previ- 
ously negated terms to continue with this line helicopter dynamic analysis. The absence of experimental data will not allow for validation of the newly generated dynamic equation. The use of traditional helicopter dynamic analysis is discouraged by number of terms that must be re-evaluated for importance. Multi-body system simulation is chosen instead, due to the ability to fully treat the coupling between the rotor blades and the fuselage and its simple implementation.

\subsection{Multi-Body System Simulation}

Multi-body systems simulations are built from basic dynamics. The method can generate and simulate the equations for an arbitrary number of bodies and joints. The equations are generated in the form most easily manipulated for numerical integration, not the minimal representation used by analytical dynamic analysis. Von Schwerin[36] discusses in-depth the advantages of matching the structure of the dynamics equations to the numerical solver. These options in-

clude changing the coordinate system, the variables used and the formalism of numeric vs symbolic. Theory of multi-body system simulation will be considered beyond the scope of this thesis. MathWork's SimMechanics is the multi-body system simulator used. MBSSIM requires models for all physics beyond body dynamics and gravity. This required development of an aerodynamic model.

\subsection{Aerodynamic Model}

A discrete aerodynamic model was developed to account for the aerodynamic effects experienced by helicopter rotor blades. Different aerodynamic models will be discussed first. Each model can be used to simulate a different aerodynamic effects. The chosen aerodynamic model will be discussed in-depth.

Momentum theory[46] is the simplest method. Momentum theory uses the actuator disk assumption and the principle of conservation of momentum to calculate the power for a given thrust. This is a one-dimensional method and doesn't 
account for variation in span-wise blade parameters or a complex rotor wake environment.

The second method is blade element theory, which discretizes a rotor blade and integrates the lift and drag over the entire blade based on the local blade element conditions. The span-wise airfoil geometry can be varied at each blade element. Classical implementation of the method achieves a commanded thrust by iterating on geometric twist/pitch. Empirical inflow equations are used to model the effects of flow through the rotor as a function of thrust. Ground effect is implemented in blade element theory by altering the local span-wise or global power due to induced drag.

These two method are combined to create a third aerodynamic model. Combined Blade element-momentum theory uses momentum theory to model inflow effects. It can still model non-constant span-wise airfoil geometry. Combined blade element-momentum theory(BEMT) rotor aerodynamic analysis will be used in the work presented below, due to its ease of implementation and validated results[17]. BEMT cannot model complex wake effects, such as, trailing tip vortices.

Vortex methods use discrete vortex filaments that trail from the rotor blade to mimic complex airflows present in the rotor wake. Gilad applied prescribed wake vortex method to the HPH rotors. Gilad concluded vortex methods don't produce results that are significantly more accurate than BEMT to justify the added time in the preliminary design phase.

Computational fluid dynamics(CFD) simulations are the most complex set of modelling techniques. CFD can model very complex free wake effect in the near and far-field. CFD does require a significant amount of computation resources to implement, and validate. CFD is too expensive for this stage of the HPH design[47]. BEMT is chosen as the aerodynamic model used for simulation of the dynamics of the HPH in a multi-body system simulation. The methodology for determining the aerodynamic forces in the MBSSIM are presented in the next section. 


\subsubsection{Methodology}

MBSSIM discretizes a vehicle body into a finite number of elements, which is used for implementation of the BEMT model. This aerodynamic model formulation can be found in Leishman[46], Bramwell[7], Padfield[34], or Johnson[43].

Blade element properties, local linear and angular velocity and the linear displacement from the ground are the inputs to the aerodynamic model. The aerodynamic model will calculate the resultant aerodynamic forces in the local blade element coordinate system.

Lift curve slope, parasite drag coefficient, chord length, reference area, and pitch angle are the local blade properties required for each element. Blade radius, and the total number of blades are global rotor properties required for each element.

A solution requires fixed point iteration on pitch to achieve the commanded thrust in traditional formulation of BEMT. Pitch/geometric twist angle is commanded at each span-wise location, making thrust and power an output in the MBSSIM formulation. This method is similar to experimental procedure rather than computer simulation. The inflow velocity is calculated using the inflow ratio defined as

$$
\lambda=\frac{v_{i}}{\Omega R}
$$

The inflow ratio calculation is described in section 2.4.2. The inflow velocity and the vehicle vertical velocity are used to find the local vertical velocity at the blade element, shown as

$$
U_{P}=\lambda(r) \Omega R+V_{c}
$$

The local horizontal velocity is an input to the aerodynamic model implemented in MBSSIM. The local horizontal velocity for a rotor in hover is calculated as 


$$
U_{T}=\Omega R(r)
$$

Sideslip forces are generated from velocity in the span-wise direction. Sideslip forces are assumed to have little or no effect. Sideslip velocity is used in calculation of velocity magnitude,

$$
U=\sqrt{U_{T}^{2}+U_{R}^{2}+U_{P}^{2}}
$$

The inflow angle is calculated as

$$
\phi=\tan ^{-1}\left(\frac{U_{P}}{U_{T}}\right)
$$

The inflow angle is used to determine the local angle of attack of the rotor blade by

$$
\alpha=\theta-\phi
$$

The coefficient of lift and drag are calculated and dimensionalized to give the forces in the wind axes in equations 2.41 through 2.43. The lift and drag forces act perpendicular and parallel to the velocity vector, respectively.

$$
\begin{gathered}
C_{l}=C_{l_{\alpha}} \alpha \\
L=\frac{1}{2} \rho U^{2} S C_{l} \\
D=\frac{1}{2} \rho U^{2} S C_{d}
\end{gathered}
$$

The method for calculation of the drag coefficient requires more detailed explanation. Assuming a drag polar equation is the simplest method for drag 
coefficient calculation. A common form is

$$
C_{d}=C_{d o}+K C_{l}^{2}
$$

The coefficients, $K$ and $C_{d o}$, are the induced drag coefficient and the parasite drag coefficient, respectively. A common empirical equation for $K$ is

$$
K=\frac{1}{\pi e A R}
$$

Interpolated aerodynamic tables are the second method for calculating drag coefficient. Numerical or experimental data is used to generate the aerodynamic tables. The tables can be interpolated using the local coefficient of lift or angle of attack and Reynold's number. The drag polar equation was used in this implementation of the aerodynamic model. The value of $C_{d o}$ is calculated by averaging the zero-lift drag coefficient over the proper range of Reynolds numbers.

MBS simulation requires force input in the local body reference coordinate system. The local body coordinate system x-direction points in-plane upstream, the z-direction points perpendicular to the disc plane, and the y-direction makes an orthogonal coordinate system. The coordinate system is shown in figure 2.5. The positive z-direction is defined as away from the ground reference. The rotation of the forces from the wind axes into the local body frame is done using a rotation matrix.

Select configurations of the HPH require both counter-rotating and forward rotating rotors. This needs to be explicitly accounted for in the MBSSIM aerodynamic model. The linear and angular velocity are assumed positive. If both are negative, a signal will be sent to the rotation matrix to change the rotation such that the drag is always acting in the direction opposite the body velocity. 
The two rotation matrices are

$$
R_{m}=\left[\begin{array}{ccc}
-\cos (\phi) & 0 & -\sin (\phi) \\
0 & 0 & 0 \\
-\sin (\phi) & 0 & \cos (\phi)
\end{array}\right]
$$

and

$$
R_{m}=\left[\begin{array}{ccc}
\cos (\phi) & 0 & \sin (\phi) \\
0 & 0 & 0 \\
-\sin (\phi) & 0 & \cos (\phi)
\end{array}\right]
$$

The coordinate system transform is

$$
\left[\begin{array}{l}
F x \\
F y \\
F z
\end{array}\right]=R_{m}\left[\begin{array}{l}
D \\
0 \\
L
\end{array}\right]
$$

The modelling of reversed flow will be discussed in the next section.

\subsubsection{Flow effects}

There are three major flow effects that are dealt with in this implementation of the aerodynamic model into MBS, inflow, reversed flow, and ground effect. Inflow is the name given to vertical airflow through the rotor as a result of thrust. The inflow affects the amount of thrust the rotor can produce by effectively decreasing the blade's angle of attack. Their are three different inflow models described by Leishman. The first and simplest model assumes constant inflow proportional to the thrust coefficient and is

$$
\lambda=\sqrt{\frac{C_{\text {Treq }}}{2}}
$$

The second inflow model uses local span-wise information to determine the inflow and is 


$$
\lambda(r)=\frac{\sigma C_{l_{\alpha}}(r)}{16}\left[\sqrt{1+\frac{32}{\sigma C_{l_{\alpha}}(r)} \theta(r) r}-1\right]
$$

This model doesn't require iteration on inflow. A model that doesn't require iteration allows for simpler implementation. Each blade element's inflow calculator uses the total rotor solidity, to match the traditional implementation of BEMT.

The last inflow model accounts for tip loss effects. Tip loss is caused by the pressure differential at the rotor tip, which causes a decrease of the inflow velocity at the tip of the blade. The last inflow model makes use of momentum theory, and is

$$
\begin{array}{r}
f=\frac{N_{b}}{2}\left(\frac{1-r}{\lambda(r)}\right) \\
F=\frac{2}{\pi} \arccos \left(e^{-f}\right) \\
\lambda(r)=\frac{\sigma C_{l_{\alpha}}(r)}{16 F}\left[\sqrt{1+\frac{32 F}{\sigma C_{l_{\alpha}}(r)} \theta(r) r-1}\right]
\end{array}
$$

The aerodynamic model implemented here uses the second inflow model.

Reversed flow is a physical event when the airflow over a section of the rotor changes direction. A traditional helicopter rotor experiences reversed flow in high forward velocity flight regimes, where the forward velocity exceeds the rotor rotation speed near the root of the blade. This event is a special case for traditional helicopters and is usually ignored. The rotor rotational speed is slower and well within reach of the HPH's angular velocity. Reversed flow must be accounted for in the aerodynamics model.

If reverse flow is found the blade section is assumed to be stalled. A stalled blade section will generate no lift, and will generate drag in the opposite direction.

Ground effect occurs when a lifting surface nears the ground. Ground effect is 
modelled by a proportional decrease in the induced power required for a constant thrust. Ground effect is experienced by both non-rotating and rotating lifting surfaces. Traditional helicopter experimental data regarding ground effect is not in the proper flight regime for the HPH. The ground effect coefficient is defined as

$$
k_{G}=\frac{P_{I G E}}{P_{O G E}}
$$

This ground effect coefficient is applied per-radial station to the inflow ratio,

$$
\lambda_{I G E}(r)=k_{G}(r) \lambda_{O G E}(r)
$$

The ground effect coefficient data is traditionally plotted against the distance from the ground non-dimensionalized by the rotor radius, or $\frac{z}{R}$. The minimum value of $\frac{z}{R}$ for traditional helicopters is 0.46 to 0.61 . This contrasts with the maximum value for a HPH of 0.46. Traditional ground effect experimental data or analytical equations are not valid for the purposes of this aerodynamic model.

University of Maryland's(UMD) Gamera project generated experimental data for ground effect in the proper flight regime. The experiment used the UMDs ground effect test rig (GETR), discussed in [17], [48], and [49]. The GETR is a $1.37 \mathrm{~m}$ sub-scale test rotor which is moved translated vertically from a $z / R$ value of 2 to .1. The thrust from the rotor and the power required by the motor were recorded. A curve fit is applied to the ground effect coefficient data. Ground effect becomes negligible as the rotor gets further from the ground, so a horizontal asymptotic function was chosen for the curve fit. The horizontal asymptote for $k_{G}$ is 1 as the height ratio goes to positive infinity. The data shows that the ground effect factor becomes negligible at a height ratio of 2 . This experimental data and the curve fit are shown in figure 2.6. 


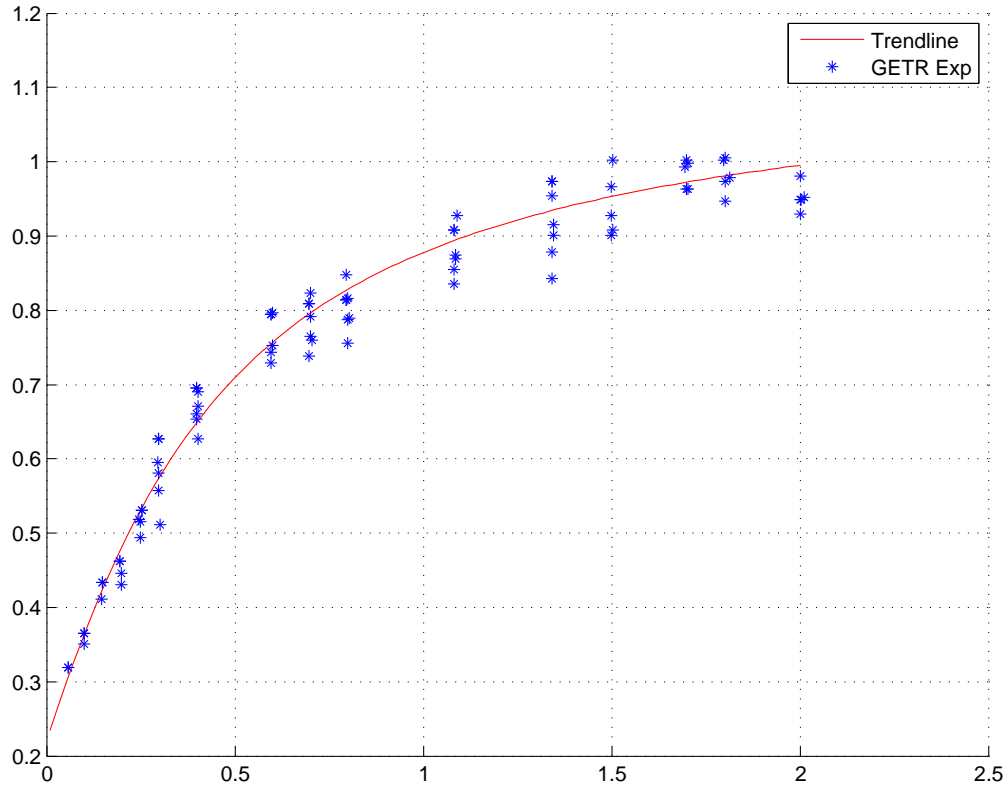

Figure 2.6: Ground Effect Data from UMD along with the corresponding trendline

The theory behind the aerodynamic model has now been presented. The model will be verified and validated in section 3.4.

\subsection{System Identification}

System identification will be used to generate a linear state space model. The inner workings of system identification and its associated methods are discussed in detail in Van Overschee[41]. System identification will be treated as a black box in this thesis. The input form, options available to the user and, the form and quality of the output will be discussed. System identification is the process of using time response data from a simulation to generate a mathematical model. The implementation of system identification using a commercial computer program will be described in chapter 3 .

The time dependent dynamic states of the system are referred to as the output. The state space model that is product of system identification is referred to as the identified model. The time-dependent data that is used as an input to the 
system is referred to as the input data. The instantiated object fed into the system identification function is referred to as the input object.

Numerical integration algorithms use adaptive time stepping to resolve areas with larger derivative. Uniform time step interpolation must be performed to make a uniform time step over the data set. Uniform time step is a requirement of the specific system identification algorithm. The time response data is assumed to be of sufficient resolution to apply a linear interpolation without any loss of data characteristics.

\subsection{Stability}

System identification was used to generate a linear state space model such that the vast literature surrounding linear system stability can be brought to bear on the problem[50]. The stability of the system can be evaluated through linear stability analysis theory. This section begins with a discussion of the solution to the linear state space system. The definition of stability will then be discussed. Finally, the types of stability and indications of stability will be discussed.

The identified system is a linear discrete-time state space system. The current state vector $x(k)$ is

$$
\begin{aligned}
k=k_{o}: \quad x(k+1)= & A(k) x_{k}+B(k) u(k) \\
k=k_{o}+1: \quad x(k+1)= & A(k) x_{k}+B(k) u(k) \\
= & A\left(k_{o}+1\right) A\left(k_{o}\right) x_{o}+A\left(k_{o}+1\right) B\left(k_{o}\right) u\left(k_{o}\right)+ \\
& B\left(k_{o}+1\right) u\left(k_{o}+1\right) \\
k=k_{o}+2: \quad x(k+1)= & A(k) x_{k}+B(k) u(k)
\end{aligned}
$$

Using inspection, the solution reduces to 


$$
x(k)=A^{k} x_{o}+\sum_{j=0}^{k-1} A^{j} B u(k-j-1)
$$

, if the system is time-invariant.

The complete solution, $y(k)$, is

$$
y(k)= \begin{cases}C x_{o}+D u\left(k_{o}\right) & , k=k_{o} \\ C A^{k} x_{o}+\sum_{j=0}^{k-1} C A^{k-j-1} B u(j)+D u(k) & , k \geq k_{o}+1\end{cases}
$$

Rugh[50] defines stability as involving the boundedness properties and asymptotic behavior of a system. Linear systems can either be classified as exponentially stable, or unstable. The proof for stability and all involved theorems will not be presented in this thesis.

A system is exponentially stability, if there exist a finite positive constant $\gamma$ and a constant $0 \leq \lambda \leq 1$ such that for any $k_{o}$ and $x_{o}$ the solution presented above satisfies

$$
\|x(k)\| \leq \gamma \lambda^{k-k_{o}}\left\|x_{o}\right\|, \quad \text { for } \quad k \geq k_{o}
$$

This definition can be applied to the state equations, without assessing the solution to the state equations, through the calculation of the eigenvalues. The eigenvalues are calculated for the discrete time case through a z-transform on the zero-input state equation and are the roots of

$$
\mathbf{Z}\left[A^{k}\right]=z(z I-A)=\frac{z \cdot \operatorname{adj}(z I-A)}{|z I-A|}
$$

A linear time invariant state equation is exponentially stable if and only if all eigenvalues of $\mathrm{A}$ have magnitude strictly less than 1 , is the key result. If this 
condition is not satisfied, the solution will identified by default as unstable. In the unstable case the solution is assumed to grow without bounds.

\subsection{Summary}

The theory used in this thesis is presented in this chapter. The NewtonEuler equations are presented to begin the presentation of traditional helicopter dynamic analysis. The traditional helicopter dynamic analysis focuses on the dynamic of the main rotor, specifically the flap degree of freedom. Equations of motion for the flap degree of freedom are derived with aerodynamic, structural and fuselage motion effects. The quasi-steady assumption is applied to decouple the rotor and fuselage interaction. The quasi-steady assumption cannot be applied to the HPH due to similarity in fuselage and rotor response times. This breakdown creates the need for a different method for HPH dynamic analysis. This alternate method is chosen to be Multi-Body System Simulation.

MBSSIM uses a set of bodies and joints to automatically generate the equations of motion for a system. These equations of motion can then be numerically integrated to generate a time response for the system. MBSSIM requires models

for all physics beyond body dynamics and gravity. This required development of an aerodynamic model. The theory for the aerodynamic model is presented in this chapter.

MBSSIM and aerodynamic theory are used to generate dynamic time response data. The time response data is processed and used for system identification. System identification generates a linear discrete time state space equation. The linear discrete time state space system is found to be to be stable or unstable thus identifying the time response data as stable or unstable. The method for determining the stability of a linear discrete state space system is presented in this chapter . 


\section{Chapter 3}

\section{Implementation}

The implementation of the models is explained here. Simulink ${ }^{\circledR}$ is a GUI, which uses blocks to represent mathematical processes applied to a time depen-

dent signal. The SimMechanics toolbox ${ }^{\mathrm{TM}}$ adds the ability to perform multi-body system simulations to Simulink. An example of implementation of a simple physical model will be discussed to better introduce the reader to MBSSIM. A set of object classes used to add scripting to the MBSSIM functionality were develop for the HPH application.

The implementation of the aerodynamic model is presented in this chapter. The aerodynamic model is verified by comparing rotor performance for a single flight condition using a varying number of elements. The specific method used for verification is a Richardson Extrapolation. The Richardson Extrapolation determines the numerical uncertainty by calculating the error in the model based on the change in performance for a varying numbers of elements. The Richardson Extrapolation calculates the apparent order of the aerodynamic model. An effort is made to validate the implemented aerodynamic model. The validation compares experimental data to the numerical data for equal flight conditions. 


\subsection{SimMechanics Implementation}

The SimMechanics block set contains basic bodies, joints, sensor and actuators. The body block holds user-defined mass and inertia properties. The user can define the coordinate system orientation and position. The body block can have any number of coordinate systems and can be at any orientation or distance from the coordinate system attached to the previous joint. The center of gravity position and orientation can be defined. The orientation of a coordinate system can be set through a set of Euler angles, a 3-by-3 orthogonal rotation matrix, or a 1-by-4 row vector quaternions. Joints blocks hold user-defined degrees of freedom. Rotation and translation are the two types of degrees of freedom. Joints can have a maximum of 6 degrees of freedom, 3 translation and 3 rotation. The basic body and joint block type can be copied and combined to create any physical mechanism. A body cannot be attached to another body and a joint cannot be attached to another joint. Systems can be modelled in a generalized way, or in detail depending on the level of analysis or design.

Compliant elements create motion in the system. The actuator block is the basic form of the SimMechanics compliant element. The input of a actuator block is a Simulink signal, and the output is connected to a joint or body. The body actuator block induces motion in the body through force or moments. The joint actuator block induces motion in the joint through forces, moments or the prescription of motion. The prescription of motion requires the position, velocity and acceleration of each degree of freedom actuated. Prescribed motion does not generate a reaction force or moment to counteract the motion. Prescribed motion

will not be used in the simulation of a $\mathrm{HPH}$ due to the lack of reaction forces or moments.

The sensor block is the fourth major block type. The sensor block can be used to measure the states of a joint or body. The body sensor can measure twenty-four states, linear position, linear velocity, linear acceleration, nine element rotation matrix, angular velocity, and angular acceleration. These states can be measured in the absolute coordinate system, or in the local coordinate system in which 
the sensor is attached. The joint sensor can measure the position, velocity and acceleration for each joint degree of freedom. The joint sensor can measure the reaction forces or moment over the joint. Closed loops can be develop through the use of a sensor and actuator block.

Sensors and actuator block output and input a Simulink signal, respectively. The Simulink signal can be displayed or analysed by the user using several types of output blocks. The model can be simulated to produce the time response of the configuration. This multi-body system approach to modelling complex behavior of bodies allows a reduction in the designers time spent in understanding and modelling of complex helicopter dynamics. This is appropriate for the HPH project. The downside of MBSSIM and numerical time integration is the absence of explanation for couplings. There is no equation that can be checked to determine the strength or weakness of components of the dynamic response. Sensors can be used to mitigate this downside through reaction force and torque sensing.

A sensor is attached to the rotor rotational joint to sense the reaction forces and torques across the rotor joint. These reaction forces and torques are used to calculate the power required and thrust generated.

Simulink allows the development of custom block libraries, that when used in a model, are still linked to the original library block. This link can be used to push or pull changes from or to the original library block. This feature allows for modularization and discretization. Documentation and discussion of the best practices in SimMechanics can be found in Appendix A.

Before continuing to the next section, a small example will be presented to better introduce the reader to MBSSIM. This example models a double pendulum and is taken from MathWorks $([8])$. The double pendulum can be described as having to two unique parts. First, the bar has some mass, inertia and length, and second is the joint which determines around which axis the bar can rotate. Figure 3.1 shows a simple drawing a double pendulum with the described elements. 


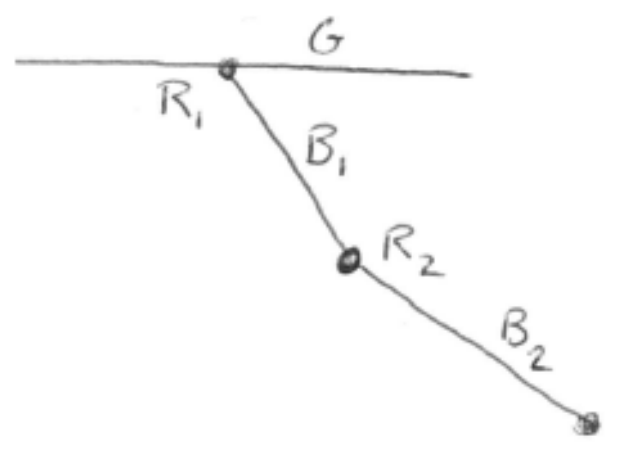

\section{Figure 3.1: Double Pendulum Drawing[8]}

These two elements of the double pendulum are described in SimMechanics as a body block and a revolute joint block. Figure 3.2 shows a SimMechanics model of the double pendulum. The red blocks represent revolute joints, and the blue blocks represent the mass and length of each aspect of the pendulum. The green block represents the fixed surface to which the double pendulum is attached. Sensor blocks and initial condition blocks are included in this model. This model can now be simulated and the system time response determined. 


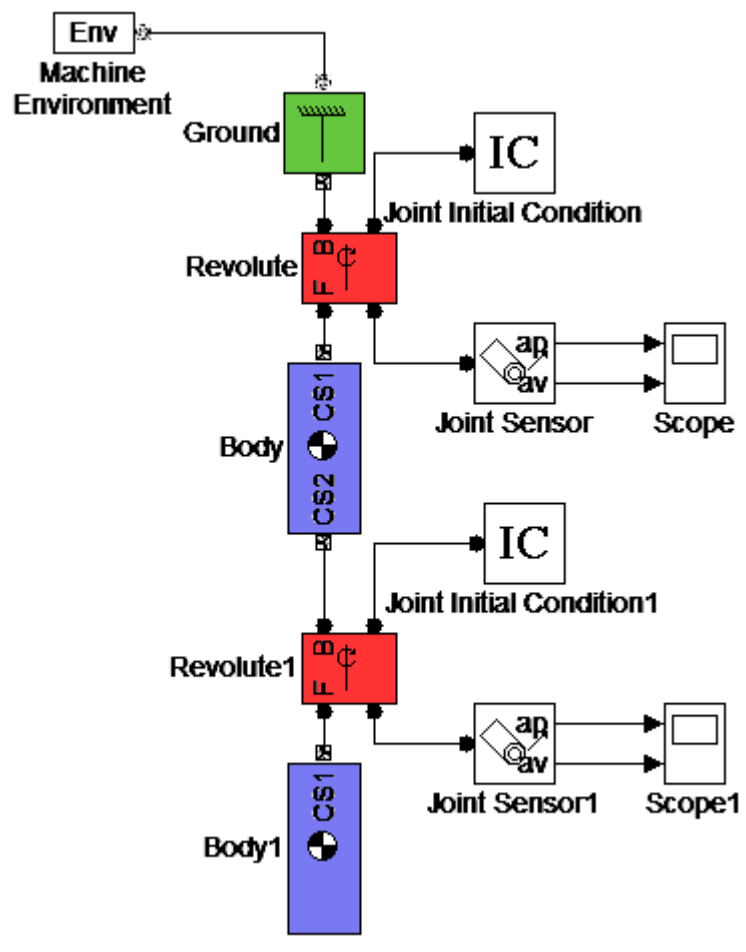

Figure 3.2: Double Pendulum Model[8]

The example above demonstrates how a physical system can be simplified and modelled in SimMechanics. This same method is used to develop the HPH models used in this thesis.

\subsection{Text User Interface}

Properties must be changed manually in normal SimMechanics modelling. The GUI aspect of SimMechanics is efficient for development of multi-body systems. SimMechanics is unable to script changes to the model. The text user interface developed for this thesis enables the scripting of SimMechanics model property changes and the recording of the time response results. This interfacing will enable the MBS to be used in design tools, such as parameter sweeps.

The text user interface is a set of object classes, which are attached to different components of the multi-body system. The Model Discovery class, and Block 
Parameter class are the two main types of object classes in the text user interface. The block parameter class contains all information about the properties of each block in the model.

Objects are instantiated and attached to specific Simulink model blocks. The attached objects query the model block for specific parameters and populate the corresponding object properties. The object and the model block are now linked, such that when a object property value is altered, the corresponding model block parameter is altered. Each block parameter object property has access methods that will control the data types and values sent to the model blocks. This is a safeguard against a common source of simulation error. The block parameter objects are sub-classed due to variation in model block parameters.

A Model Discovery class object is instantiated for each SimMechanics model that is generated. The Model Discovery object has properties linked to global model parameters. A Model Discovery object has properties that contain the figures of merit, scope data and identified system data. The Model Discovery object also contains handles for the block parameter class objects. Model Discovery class methods are used to simulate the model, process the model time response and input/output groups of model parameters.

Figure 3.3 shows a block diagram of the parameter classes and subclasses, along with their unique properties. 

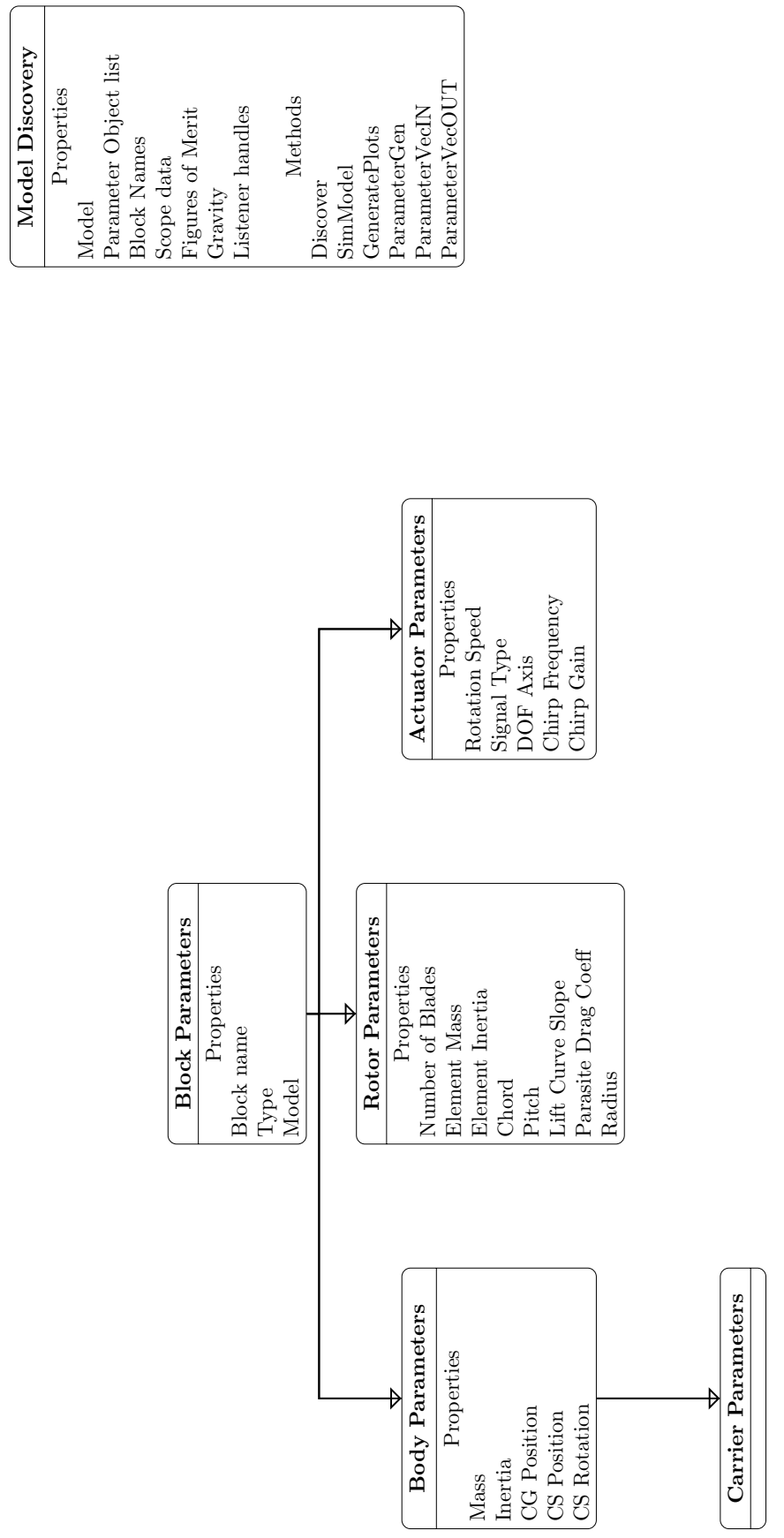

Figure 3.3: Class Diagram 


\subsection{System Identification}

The system identification toolbox ${ }^{\mathrm{TM}}$ in MATLAB ${ }^{\circledR}$ is used to perform system identification through the $\mathrm{n} 4 \mathrm{sid}$ function. This function requires the input of an iddata object, which contains time, input and output data, and the order of the identified model. The form of the identified state space model is

$$
\begin{aligned}
& x(k+1)=A x(k)+B u(k) \\
& y(k+1)=C x(k)+D u(k)
\end{aligned}
$$

The $A, B, C, D$ matrix sizes are determined by the order of the model, number of inputs, and outputs. If $n$ is the model order, $m$ is the number of system inputs, and $l$ is the number of system outputs, $A$ is $n \times n, B$ is $n \times m, C$ is $l \times n$, and $D$ is $l \times m$. The number of parameters available to the system identification algorithm is set when the model order chosen. The system identification toolbox ${ }^{\mathrm{TM}}$ implemented in MATLAB $^{\circledR}$, has a assessment and recommendation system for choosing the model order. The user is referred to the MathWorks documentation for more details[51].

\subsection{Aerodynamic Model}

In section 2.4, the theory behind the aerodynamic model was discussed. The implementation of the theory into SimMechanics is discussed in this section. The implemented model solution will be verified and validated to be accurate enough for our purposes. Code verification takes the form of debugging and error checking and will not be discussed. Solution verification has three aspects, input accuracy, numerical solution accuracy, and output data accuracy. Numerical solution accuracy will be the only aspect discussed. Validation studies measure the amount of agreement between computational results and experimental measurements. The 
theory and methods behind verification and validation studies are discussed in detail in Oberkampf[52].

The aerodynamic theory implementation is shown in figure 3.4. This model represents a feedback system meant to actuate a body element. This body element will be actuated to simulate a lifting surface element in a rotor blade. 


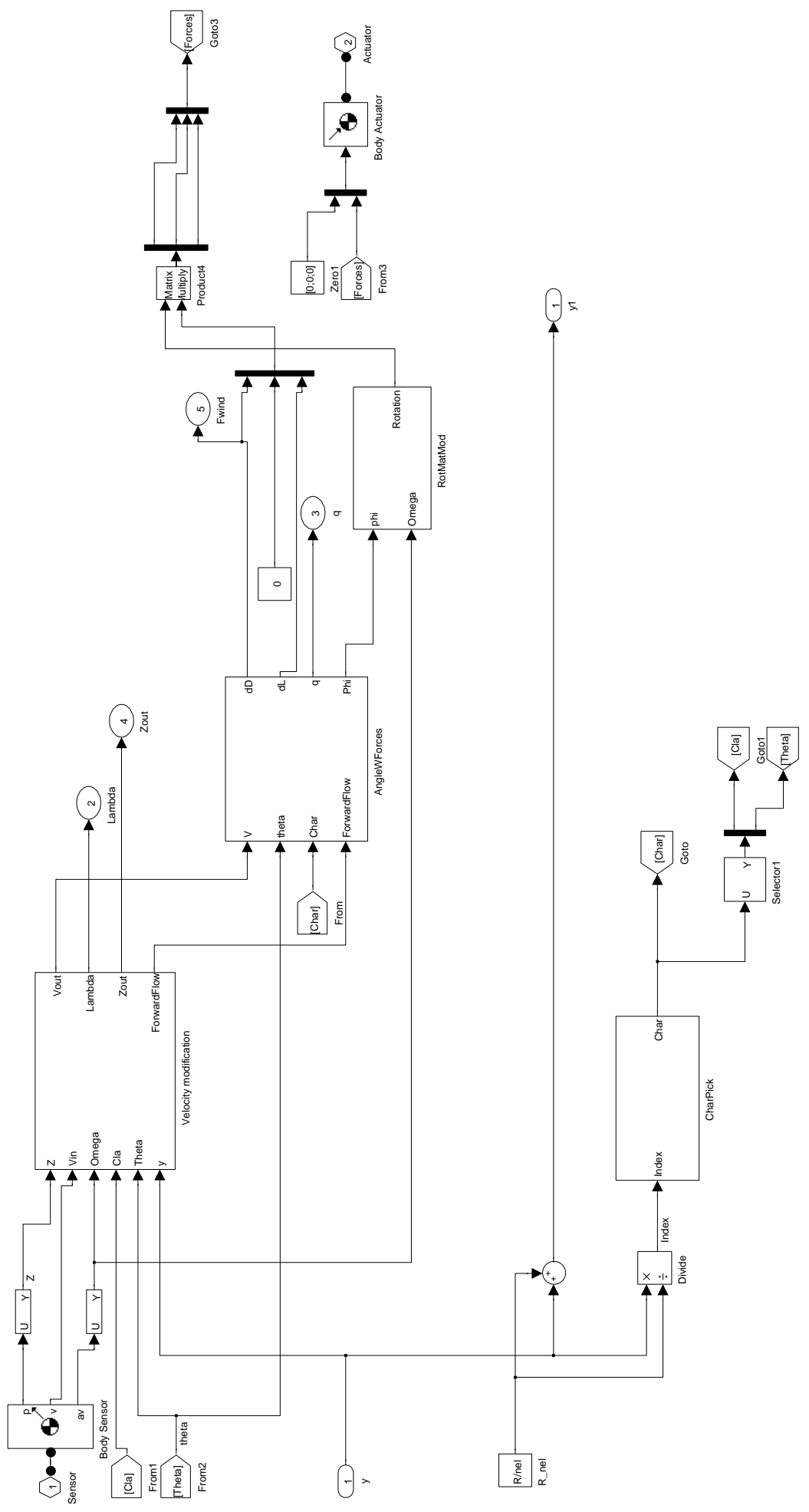

Figure 3.4: Aerodynamic model implemented in Simulink 


\subsubsection{Verification}

Numerical error estimation is the focus of the verification section. Estimation of numerical error is determined using the order of accuracy through refinement of the finite element method used in SimMechanics. A SimMechanics rotor is modelled and simulated with a varying number of body elements to assess the change in a global parameter, Power coefficient $\left(C_{P}\right)$. The parameter is assumed to converge to a value absent of numerical error as the number of elements increases toward positive infinity. Figure 3.5 exhibits this convergence.

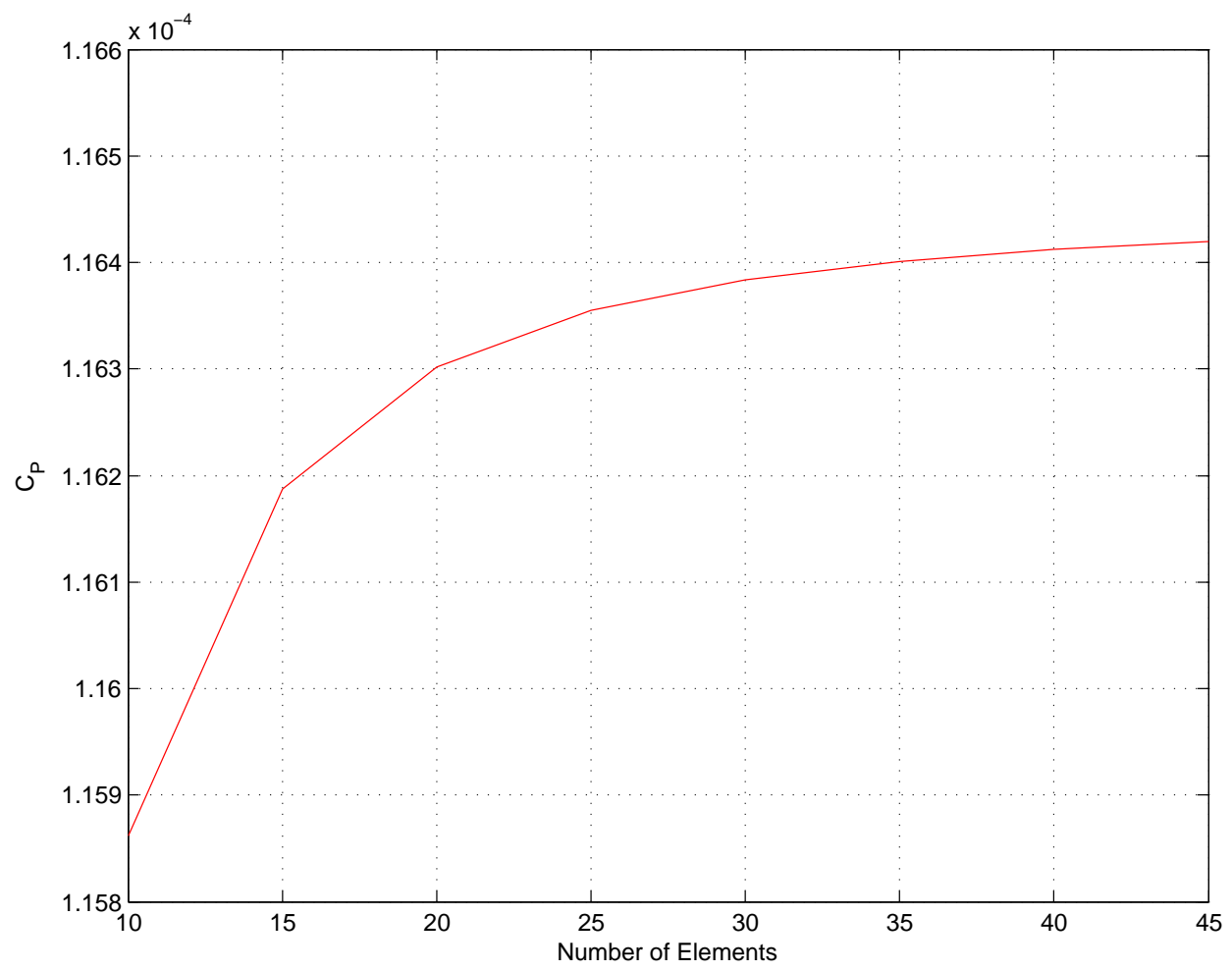

\section{Figure 3.5: Grid Refinement Study for Aerodynamic model on repre- sentative rotor}

An a posteriori error estimation was performed using the grid refinement study data to determine the observed order of the system and the estimated numerical error the figure of merit[53]. Richardson extrapolation is the specific a posteriori error estimation used. The results of this study are summarized in Table 3.1 below. Apparent order, extrapolated error, and GCI are the important figures to note. The apparent order for this study is 2nd order, indicating quadratic 
Table 3.1: Discretization Error: Richardson Extrapolation

\begin{tabular}{|l|l|}
\hline Attribute & $C_{p}$ \\
\hline \hline Number of element, $\left(N_{1}, N_{2}, N_{3}\right)$ & $45,30,10$ \\
\hline Grid refinement factor, $r_{21}$ & 1.5 \\
\hline Grid refinement factor, $r_{32}$ & 3 \\
\hline$C_{p}^{1}$ & $0.11641 \times 10^{-3}$ \\
\hline$C_{p}^{2}$ & $0.11638 \times 10^{-3}$ \\
\hline$C_{p}^{3}$ & $0.11586 \times 10^{-3}$ \\
\hline Apparent order, $p$ & 2.0001 \\
\hline$C_{p \text { ext }}^{21}$ & $0.4659 \times 10^{-4}$ \\
\hline Approximate Relative Error, $e_{a}^{21}$ & $0.31134 \times 10^{-2} \%$ \\
\hline Extrapolated relative Error, $e_{\text {ext }}^{21}$ & $149.8 \%$ \\
\hline Fine-grid convergence index, $G C I_{\text {fine }}^{21}$ & $3.1132 \times 10^{-2} \%$ \\
\hline
\end{tabular}

convergence. The extrapolated relative error is a measure of the relative error when the number of elements approaches infinity. The extrapolated relative error for the finest mesh is approximately $150 \%$. This is due to the relatively low number of elements used when compared to the order of magnitude of the power coefficient. The grid convergence index(GCI) is the ratio of the approximate relative error to the grid refinement.

Verification study indicates that the numerical uncertainty is sufficiently low when using ten elements. Ten elements will be used in the rotor validation studies and in all models used in the results section.

\subsubsection{Validation}

The aerodynamic model needs to be validated to predict realistic trends and effects, now that the model has been verified. The validation study will compare a numerical rotor model and attached aerodynamic model with experimental data of helicopter rotor. The experimental data needs to have dynamic similarity with the complete system that the tool will be applied to.

Dynamic Similarity is established by matching the Reynold's numbers at the tip of the rotor blade. Reynold's number is given by 


$$
R E=\frac{V c}{\mu}
$$

, where $\mu$ is the kinematic viscosity, and is assumed to be $1.5236 \times 10^{-5} \frac{\mathrm{m}^{2}}{\mathrm{sec}}$. Table 2.1 details the Tip Reynold's number ranges for the historical HPHs. The validation data selected to match these ranges comes from the University of Maryland(UMD), where the recent development of the Gamera has produced a large amount of experimental data on low-Reynolds number rotor performance.

Table 3.2: Experimental Test Description

\begin{tabular}{|l|l|l|}
\hline Attribute & UMD GETR & UMD FSTR \\
\hline \hline Radius $(\mathrm{m})$ & 1.37 & 6.5 \\
\hline Chord $(\mathrm{m})$ & .27 & 1 \\
\hline Twist & none & none \\
\hline Taper & none & none \\
\hline Reynolds Number Range $\left(\times 10^{5}\right)$ & $1.37-2$ & $5-9$ \\
\hline Airfoil & NACA 0012 & Eppler 387 \\
\hline
\end{tabular}

The two experimental setups used for validation data are the sub-scale ground effect test rig(GETR), and the full scale HPH blade test rig(FSTR), which appear in [17] and [49]. The UMD Full Scale test rig experiences ground effect, and for this reason the GETR was included to validate the aerodynamic model out-of-ground effect(OGE). Table 3.2 lists the characteristics for each experimental setup. A SimMechanics model was built with specifications for each UMD experimental setup used to record the validation data.

Figures 3.6 through 3.9 show comparison of the SimMechanics aerodynamic model and GETR experimental with a variation of rotor rotation speed. Figures 3.10 and 3.11 show a comparison of the SimMechanics model and the Full scale test rig with variation in rotor height above ground. The dashed line in each figure represents the numerical aerodynamic model results from Gilad[17], and are presented for comparison.

The SimMechanics Model in figure 3.6, shows good correlation with the experimental data and numerical simulation performed by Gilad. The last data point in the figure shows the start of stall as a higher thrust is commanded. The 
SimMechanics model did not account for stall at high angles of attack. The SimMechanics model consistently over-predicts the coefficient of power compared to Gilad's numerical simulation.

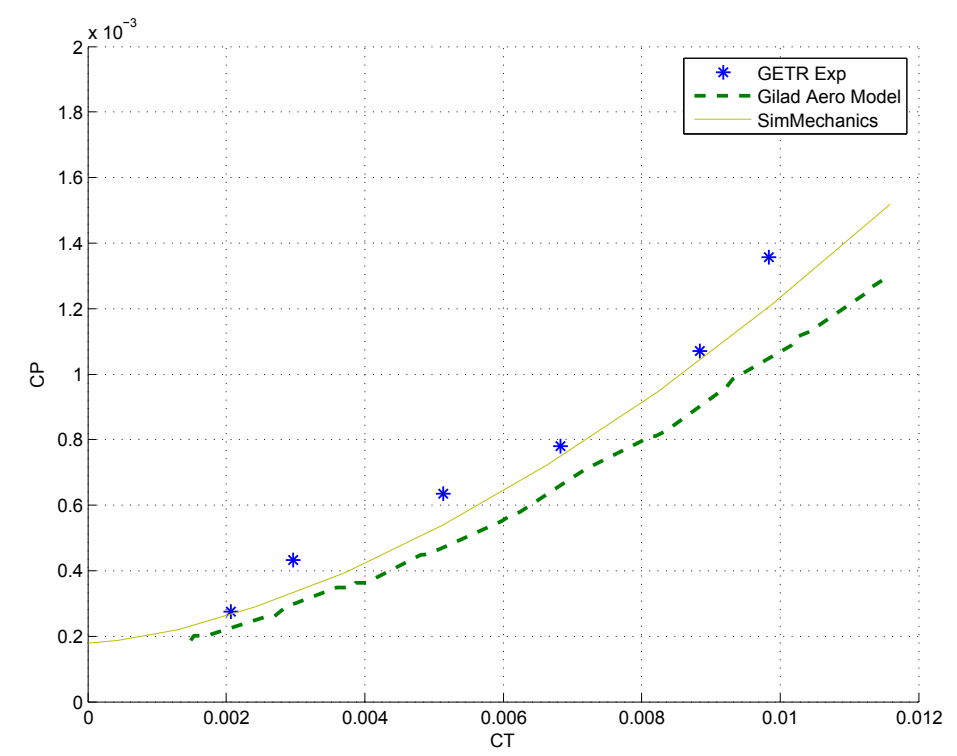

Figure 3.6: SimMechanics Validation using UMD GETR data $\Omega=$ 54 rpm $z / R=2$

Figure 3.7 shows slightly better correlation between the experimental data and the SimMechanics model. The SimMechanics model still over-predicts the coefficient of power compared to Gilad's numerical simulation. 


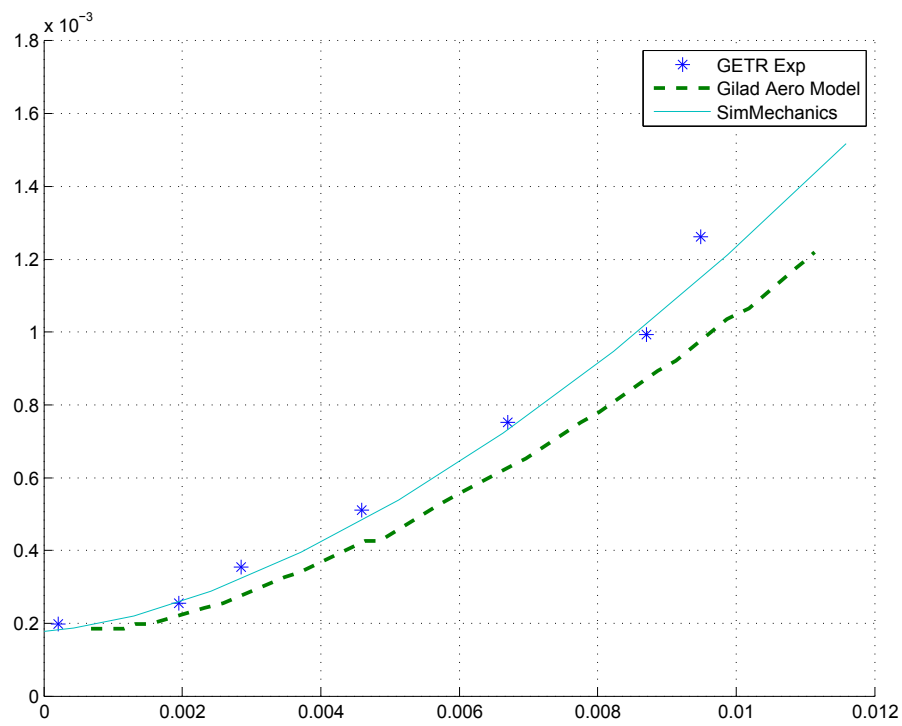

Figure 3.7: SimMechanics Validation using UMD GETR data $\Omega=$ $66 r p m z / R=2$

Figure 3.8 an under-prediction of the coefficient of power compared to the experimental data. The SimMechanics model, again, shows an over-prediction compared to Gilad's numerical simulation. 


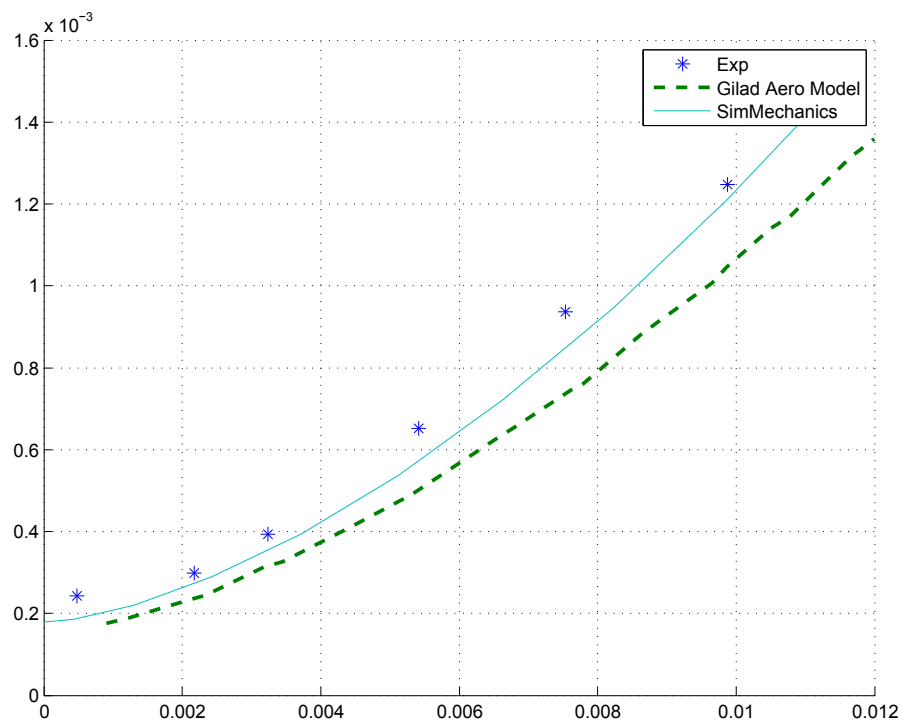

Figure 3.8: SimMechanics Validation using UMD GETR data $\Omega=$ $78 \mathrm{rpm} \quad z / R=2$

Figure 3.9 shows the SimMechanics model agains the experimental data published by the UMD. This data is taken at 82 RPM, which corresponds to a Reynolds number of $2.08 \times 10^{5}$. The SimMechanics model under-predicts the coefficient of power compared to the experimental data. The SimMechanics model over-predicts the coefficient of power compared to Gilad's numerical simulation. 


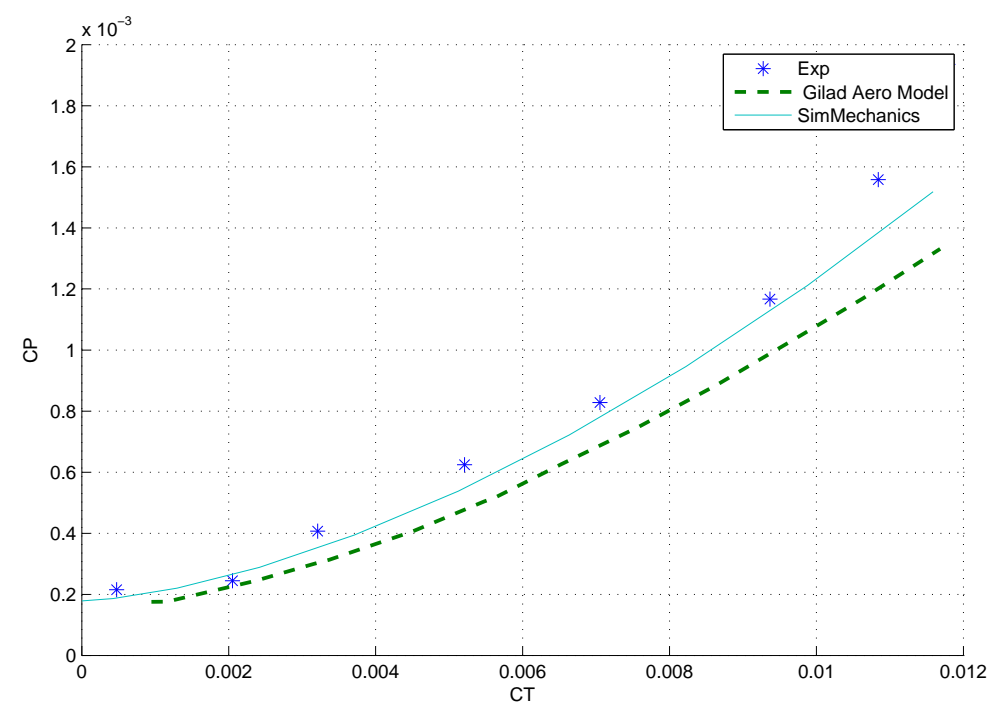

Figure 3.9: SimMechanics Validation using UMD GETR data $\Omega=$ $82 \mathrm{rpm} \quad z / R=2$

Figure 3.10 shows the SimMechanics rotor model compared to the experimental data collected from the UMD's full scale test rig. The experimental data was collected at a Reynolds number of $8.04 \times 10^{5}$ and a height ratio of .1 The SimMechanics rotor model over-predicts the coefficient of power at coefficients of thrust between 0.005 and 0.007 compared to the experimental data. The SimMechanics model has low error when compared to the experimental at coefficient of thrust numbers above 0.008 . 


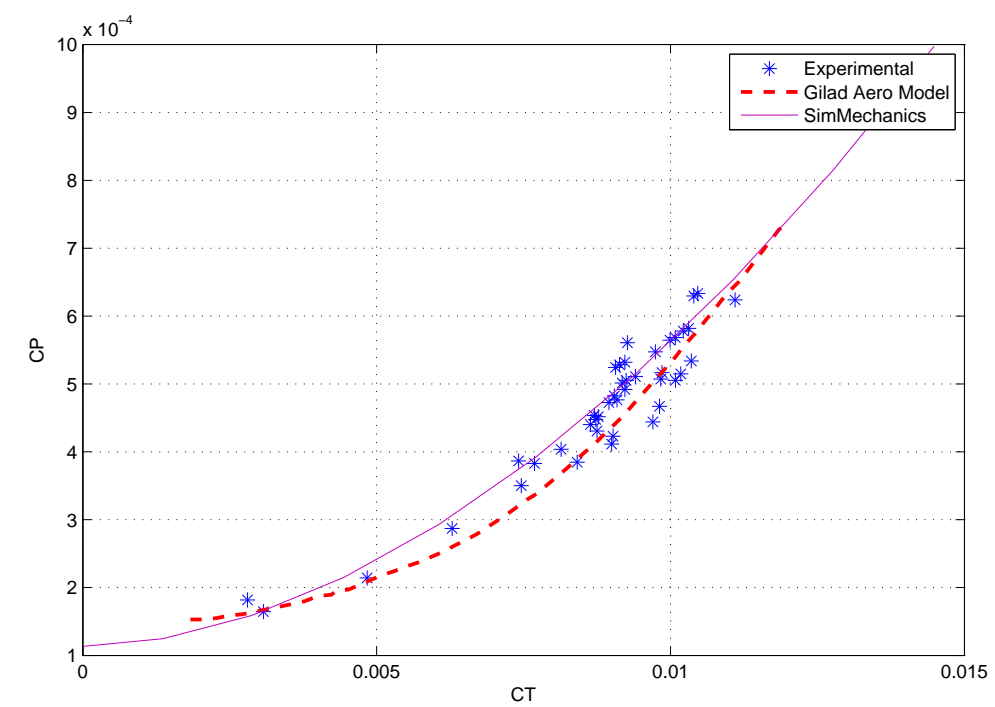

Figure 3.10: SimMechanics Validation using UMD FSTR data $\Omega=$ $18 \mathrm{rpm} \quad z / R=.1$

Figure 3.11 shows the SimMechanics rotor model compared to the experimental data collected from the UMD's FSTR. The experimental data was collected at a Reynolds number of $8.04 \times 10^{5}$ and a height ratio of .2. The SimMechanics model, again, over-predicts the power coefficient at mid-range thrust coefficients, and shows lower error near higher thrust coefficients. Experimental data is not given for low thrust coefficients, $C_{T}<0.007$, but the SimMechanics model does appear to have a lower zero-thrust power coefficient when compared to Gilad's numerical simulation. This under-prediction is directly correlated to the aerodynamic tables used, and the parasite drag coefficient used. 


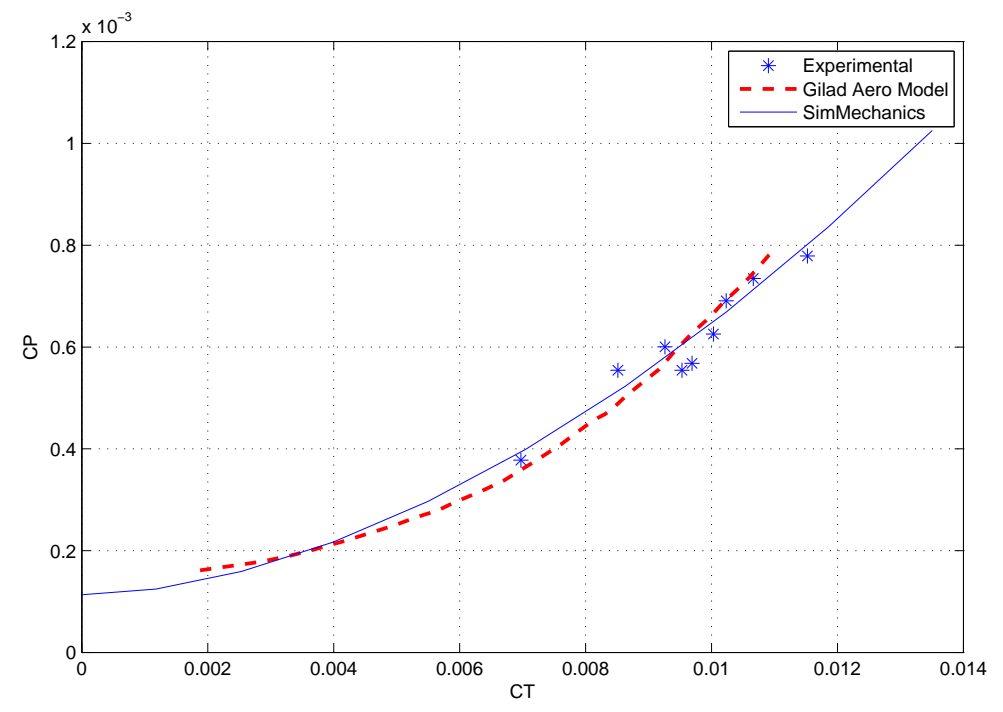

Figure 3.11: SimMechanics Validation using UMD Full Scale Test Rig data $\Omega=18 \mathrm{rpm} \quad z / R=.2$

The GETR data tests the SimMechanics model's OGE functionality, at Reynold's numbers out of the HPH range. The full scale test rig data tests the ground effect and Reynolds number correlation of the SimMechanics model. The SimMechanics model shows correlation with the experimental data, that is sufficient for use in this methods demonstration.

The validation study requires estimation of experimental uncertainties, for proper and formal validation of computational models[54]. The experimental uncertainty was not computed and presented in conjunction with the experimental data. Consequently, the models cannot be labelled as validated. The models correlate sufficiently for the purposes of this thesis, through visual inspection.

\subsection{Summary}

The implementation chapter has shown the important aspects of the SimMechanics multi-body system simulation program. The text user interface that used SimMechanics to execute simulations was discussed in this chapter. The 
text user interface enables the user to script commands. Command scripting is not available in the SimMechanics GUI. The method for system identification is treated as a black box.

The inputs and outputs to the system identification black box are discussed. System identification require the input of response data and the desired order of the linear state space system. The order was left to be chosen by the MATLAB System Identification toolbox.

The rotor aerodynamic model implemented in SimMechanics was verified through a Richardson extrapolation. The Richardson extrapolation show that the rotor aerodynamic model was second order accurate, with a low GCI percentage.

An effort to validate the rotor aerodynamic model implemented in SimMechanics is show at the end of this chapter. The UMD experimental data from the Ground effect test rig and Full scale test rig are used. Several charts are presented that compare the experimental data points to the rotor aerodynamic model trends for the two different experimental setups and several different flight conditions. The rotor aerodynamic model showed good correlation with the experimental data. The model was not validated due to the lack of experimental uncertainty data. 


\section{Chapter 4}

\section{Results}

All the components of dynamic analysis of multi-body system simulation for a HPH have been present in the previous chapters. A demonstration of the HPH multi-body system simulation will be presented in this chapter. This demonstration will discuss the implications of HPH multi-body system simulation use in design studies. This chapter will demonstrate the MBSSIM on the Da Vinci and Gamera SimMechanics models. The SimMechanics models and the SimMechanics visualization of the two configurations are shown in 4.1 and 4.3 , and 4.2 and 4.4 , respectively. 


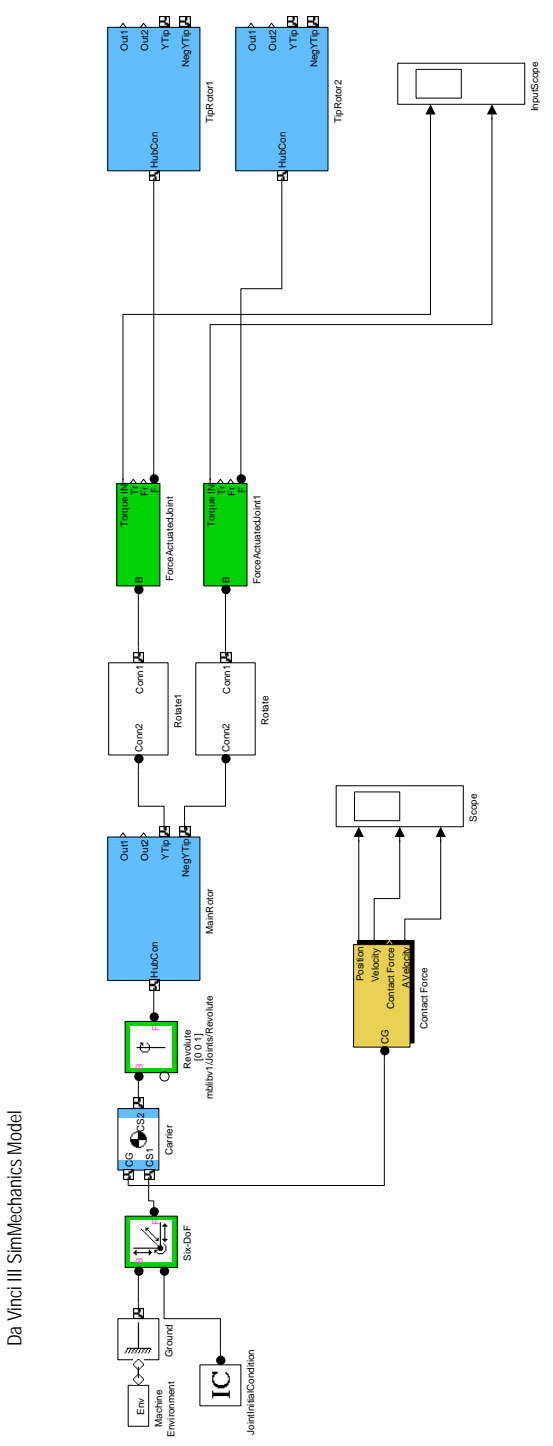

Figure 4.1: SimMechanics Model of tip rotor configuration 


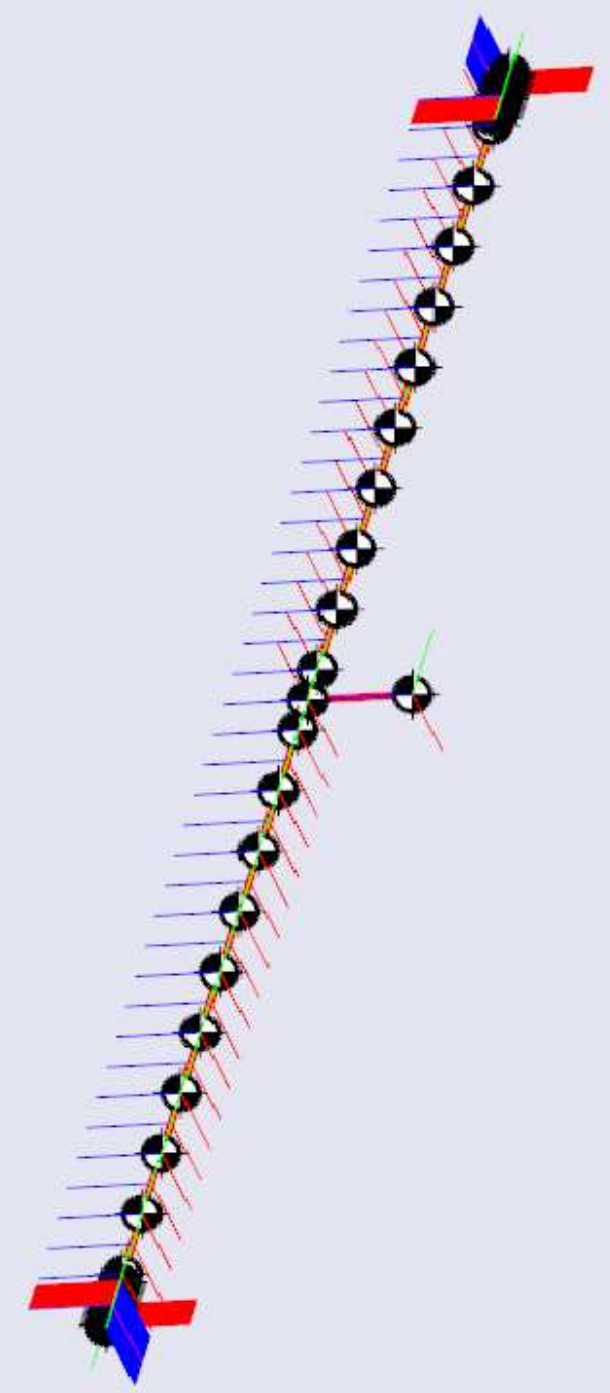

Figure 4.2: SimMechanics Visualizations of tip rotor configuration 


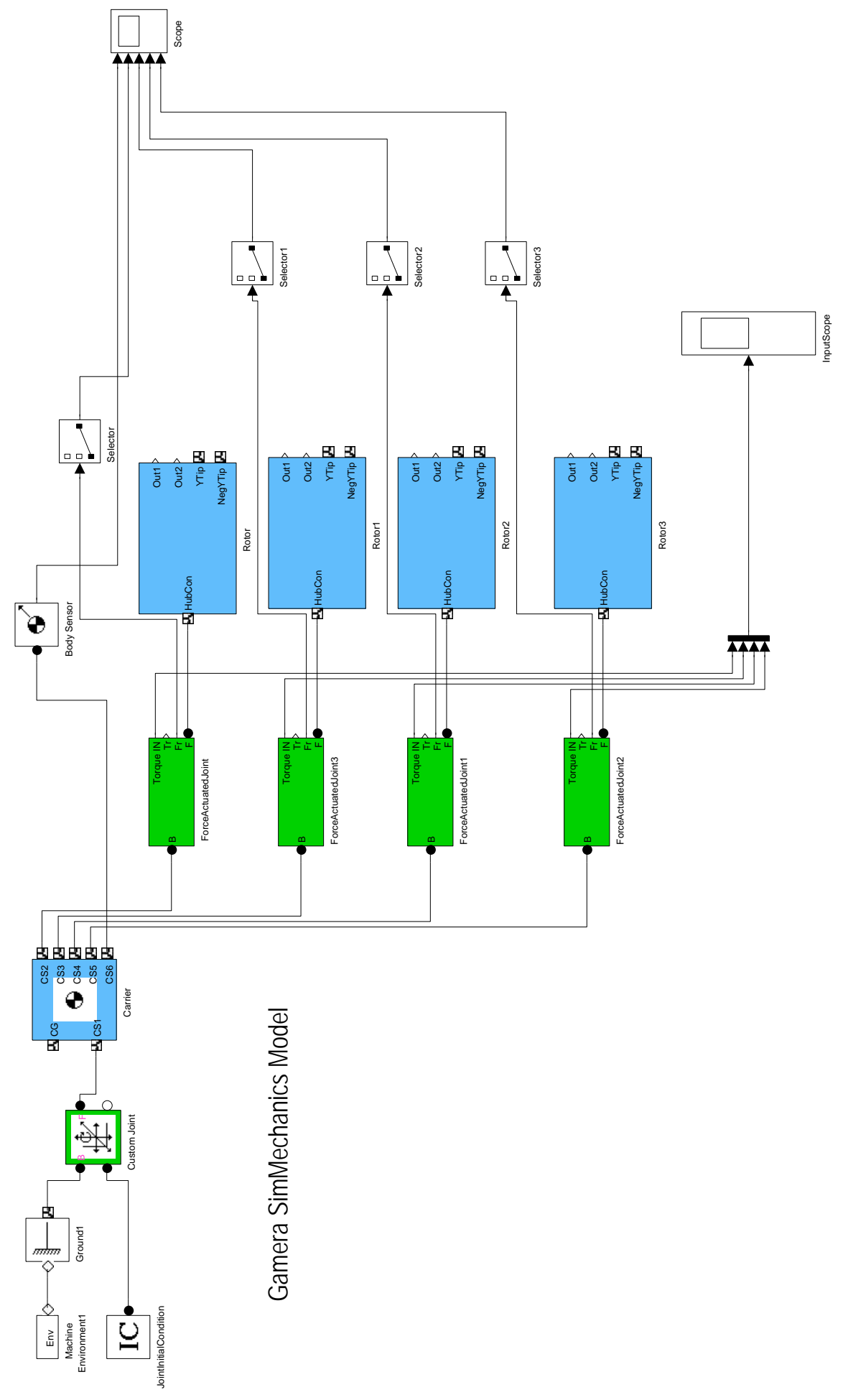

Figure 4.3: SimMechanics Model of quadrotor configuration 


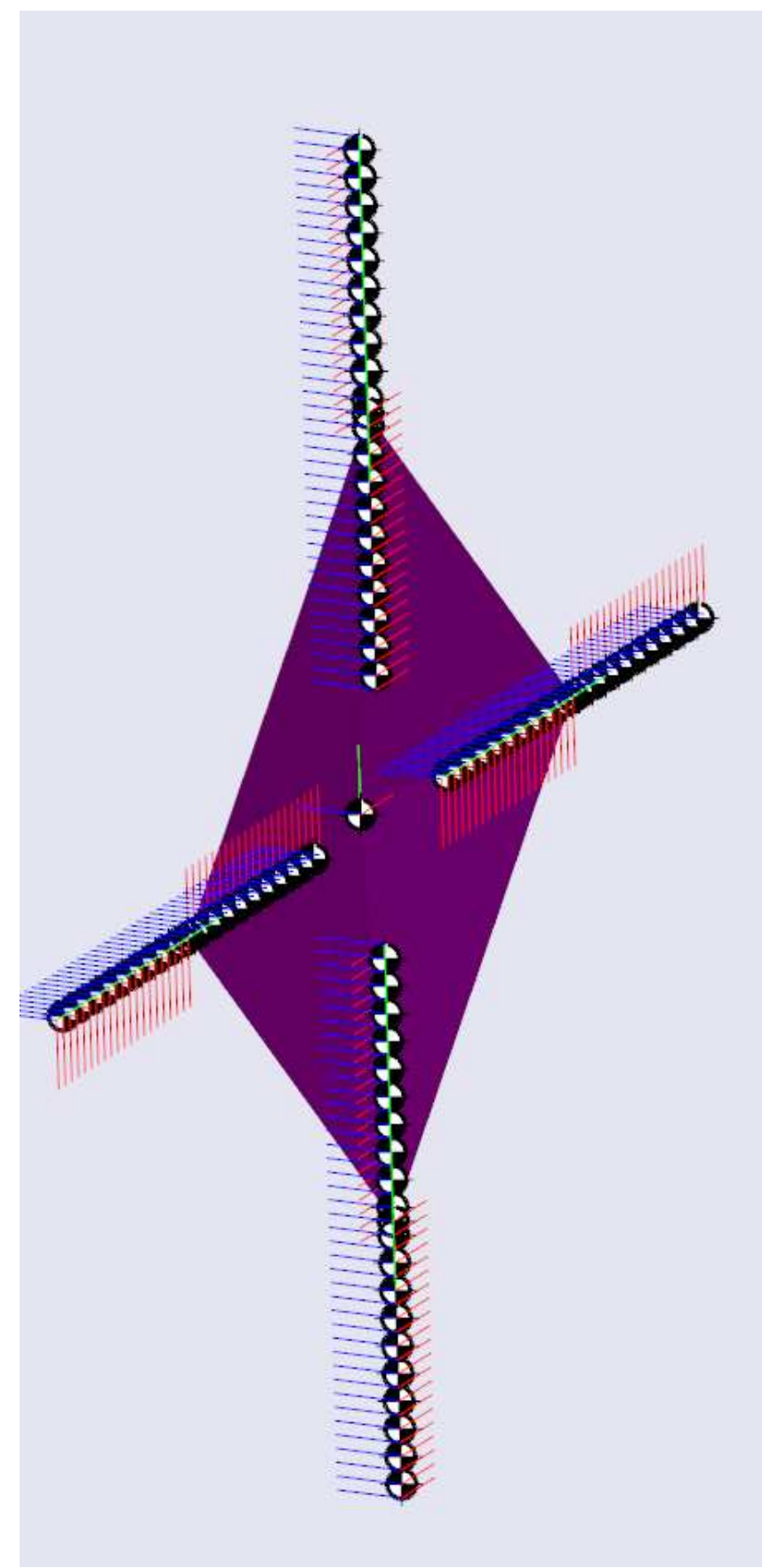

Figure 4.4: SimMechanics Visualizations of quadrotor configuration 
Due to the high instability associated with the tip propeller-driven main rotor configuration shown by the time response given in figure 4.5, the quadrotor configuration will be the focus of this chapter.
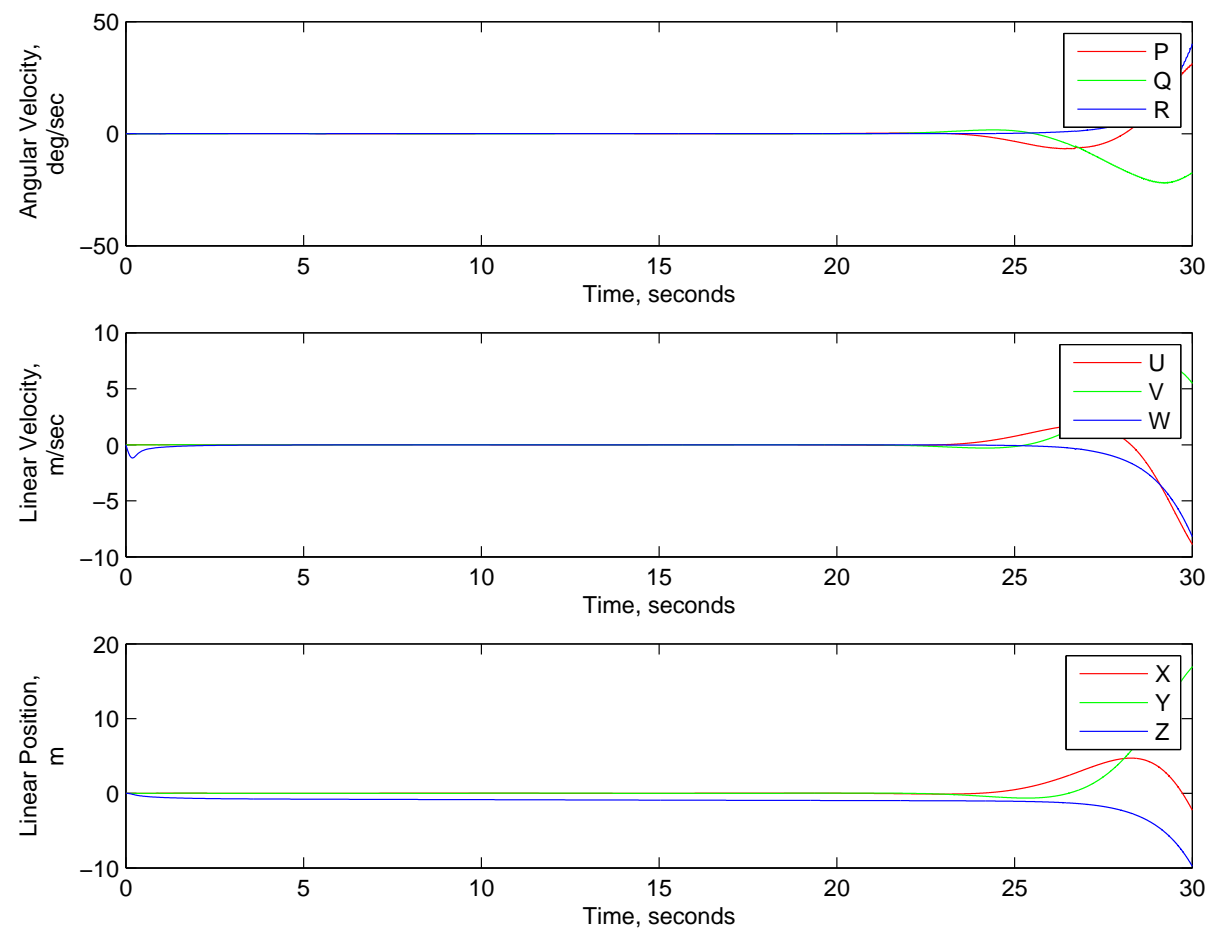

\section{Figure 4.5: Time Response for Base Da Vinci III configuration}

The base symmetric quadrotor configuration time response is presented first. The effects of varying the baseline configuration is presented second. Thirdly, the stability trends of both the Gamera and the Da Vinci configuration will be presented. The properties of the base configuration are given in Table 4.1. There is assumed to be no span-wise variation in rotor properties. Parameter produced through system identification will be discussed after time response. The carrier linear velocity and displacement, and angular velocity are used as the output data. The torque applied to each rotor is used as the input data. The model order is held at three for the stability trends; the system identification toolbox will choose the best model order for the perturbation studies. 
Table 4.1: Quadrotor Baseline configuration characteristics

\begin{tabular}{|l|l|}
\hline Characteristic & Value \\
\hline \hline Rotor Radius, $m$ & 6.5 \\
\hline Number of Elements per blade, -- & 10 \\
\hline \# of blades,-- & 2 \\
\hline Element Mass, $\mathrm{kg}$ & 0.33125 \\
\hline Blade Pitch, $\mathrm{deg}$ & 5 \\
\hline Chord, $\mathrm{m}$ & 1 \\
\hline Lift Curve Slope, $1 / \mathrm{rad}$ & 6.45 \\
\hline Lift induced drag coefficient $\mathrm{K},--$ & 0.013 \\
\hline Parasite Drag Coefficient $C_{d o},-$ & 0.01216 \\
\hline Commanded Rotation Speed, $R P M$ & 20 \\
\hline Carrier Mass, $\mathrm{kg}$ & 22.2 \\
\hline
\end{tabular}

\subsection{Time Responses}

Perturbation of the base configuration demonstrate the effect of construction tolerances. A 6-DOF joint connects the ground to the carrier, making this a 6-DOF simulation. The vehicle and its rotors, will start from rest at the beginning of each simulation. The rotor actuator uses a Proportional-IntegralDerivative(PID) controller to command the rotation speed. The PID controller will generate some wind-up time indicated by the spike near the beginning of the simulation. The controller has unlimited torque available to achieve the desired rotation speed. A human powerplant doesn't possess unlimited torque, which means these simulation are not a realistic models of piloted HPH. The vehicle will likely be falling due to the absence of a ground collusion constraint during the windup time. The simulation will run for a total of 50 seconds. The time response of the base configuration is given in figure 4.6. Figure 4.7 shows the torque applied to each rotor for the base simulation. The input torque will be similar to this for the rest of the simulations unless otherwise presented. Each case is an perturbed from the baseline and are presented in the list below.

1. Figure 4.9: increased element mass for a single rotor by $10 \%$

2. Figure 4.8: increased each term in element inertia matrix for a single rotor by $10 \%$ 
3. Figure 4.10: increased the collective pitch of a single rotor by $5 \%$

4. Figure 4.11: changed the vehicle's initial lateral attitude from zero to 5 degrees

5. Figure 4.13: increased the vehicle's initial lateral velocity from 0 to $.1 \mathrm{~m} / \mathrm{sec}$

\subsubsection{Gamera: Base Configuration}

The base configuration time response shows a quick drop and then a fast acceleration vertically to a steady state height of approximately 5 meters. There is no significant angular velocity behavior. The identified model for this simulation has both eigenvalues slightly less than one, indicating stable identified system. The torque applied to the rotors peaks at $1250 \mathrm{~N} / \mathrm{m}$. The actuator torque reaches steady state by 5 seconds. This is an indicator that the rotors have reached a steady state rotation speed. 

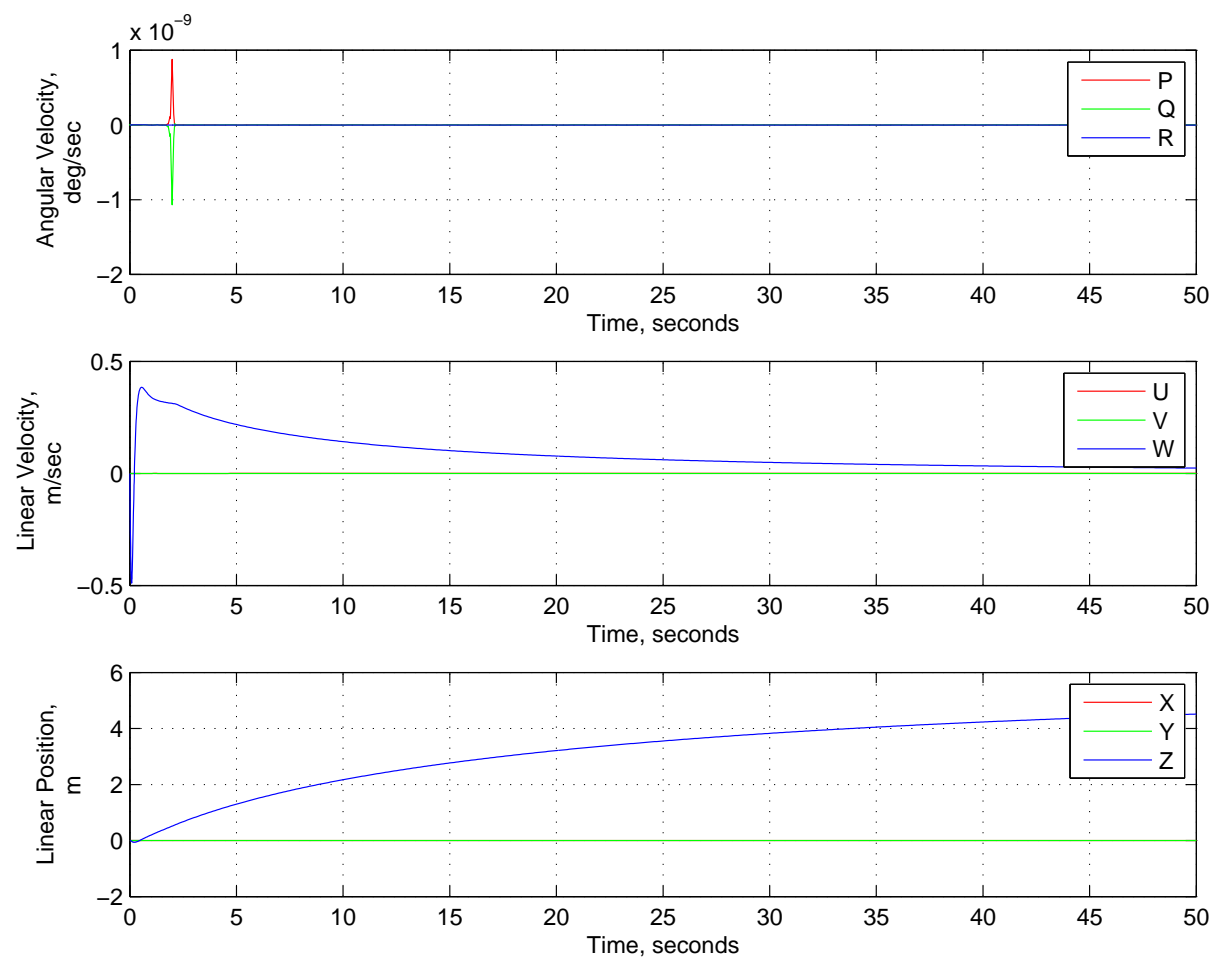

Figure 4.6: Time response for base quadrotor configuration 


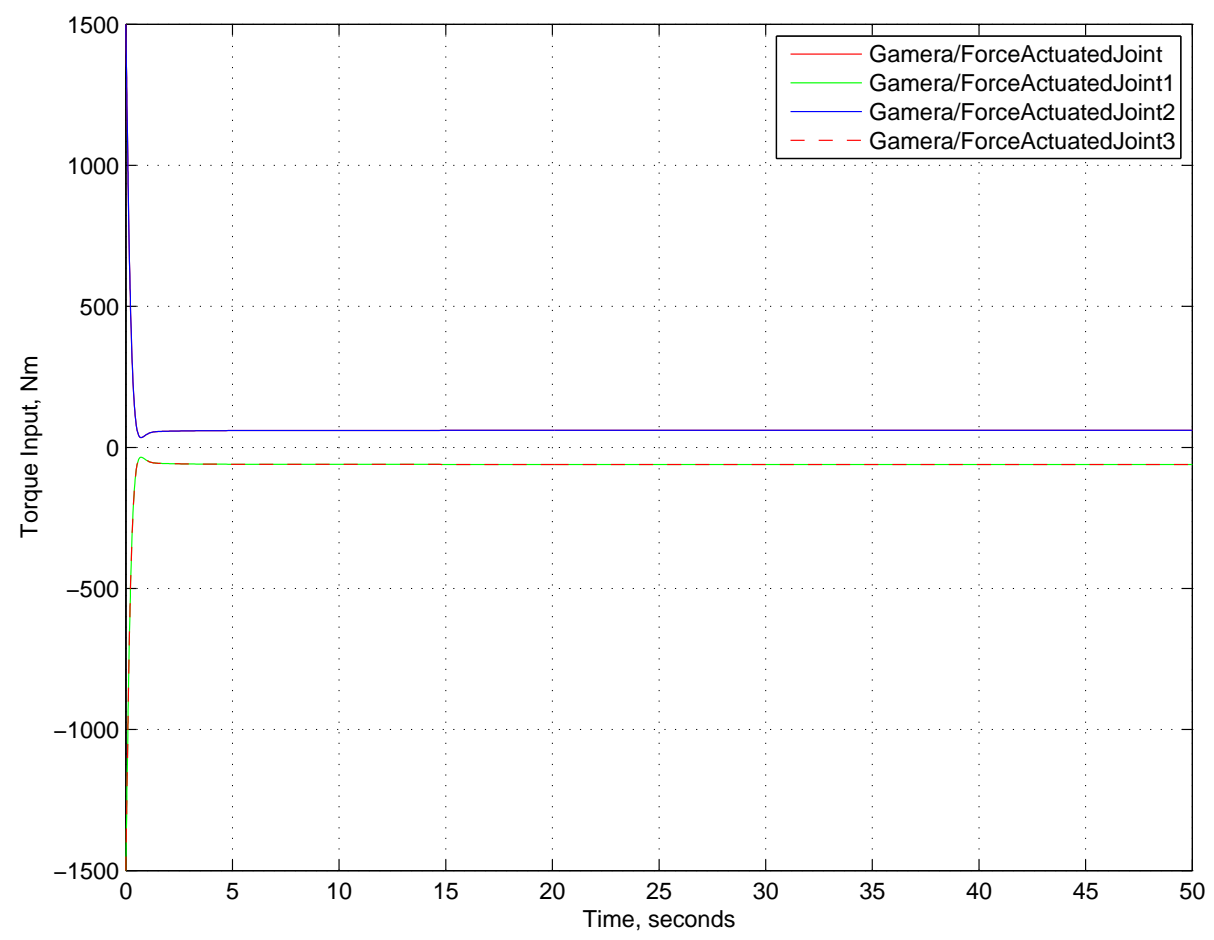

Figure 4.7: Torque applied to rotors on base configuration 


\subsubsection{Gamera: Uneven Rotor Inertia}

Figure 4.8 shows the vehicle climbs to a altitude of approximately 4 meters and hovers level with very little oscillation. The identified model, however, has one eigenvalue greater than one, indicating an unstable mode. This could be an due to the slowly increasing lateral displacement. There is no significant stability issues present in the uneven inertia case.
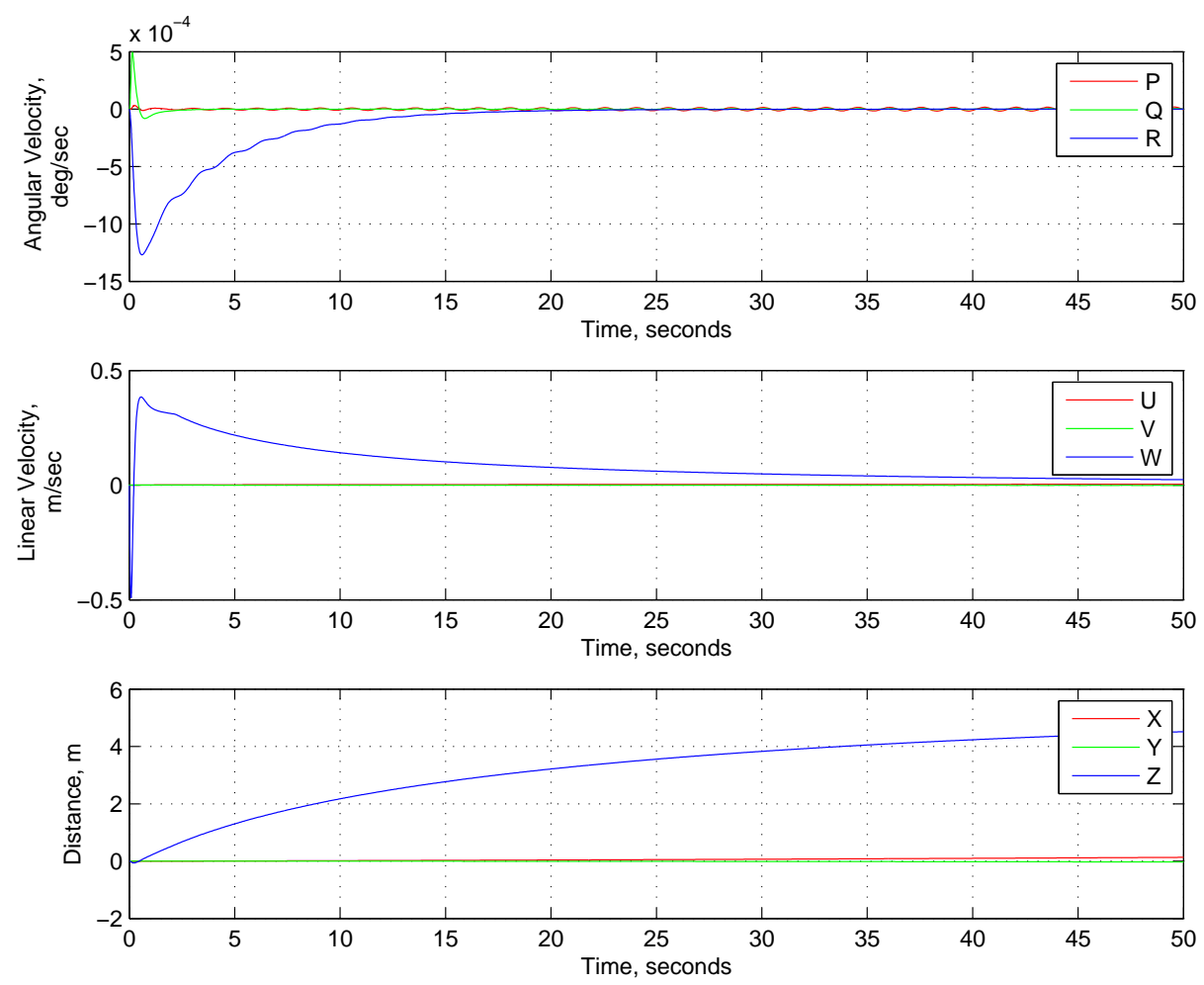

Figure 4.8: Time response for the quadrotor with a uneven inertia

\subsubsection{Gamera: Uneven Rotor Mass}

Figure 4.9 show that in the first 5 seconds the carrier has positive vertical velocity. The carrier begins to lose altitude and translate laterally. There is a divergent high frequency angular oscillations around its lateral x-axis. This behavior is similar to the crash experience by the Gamera II on 8/20/2012 shown 
in [16]. The lack of significant angular oscillation in the video as is in the simulation might be attributed to the structural damping of the fuselage frame. The identified model for this simulation has two unstable eigenvalues. The stability of the identified system matches the divergence of the time response, visually.
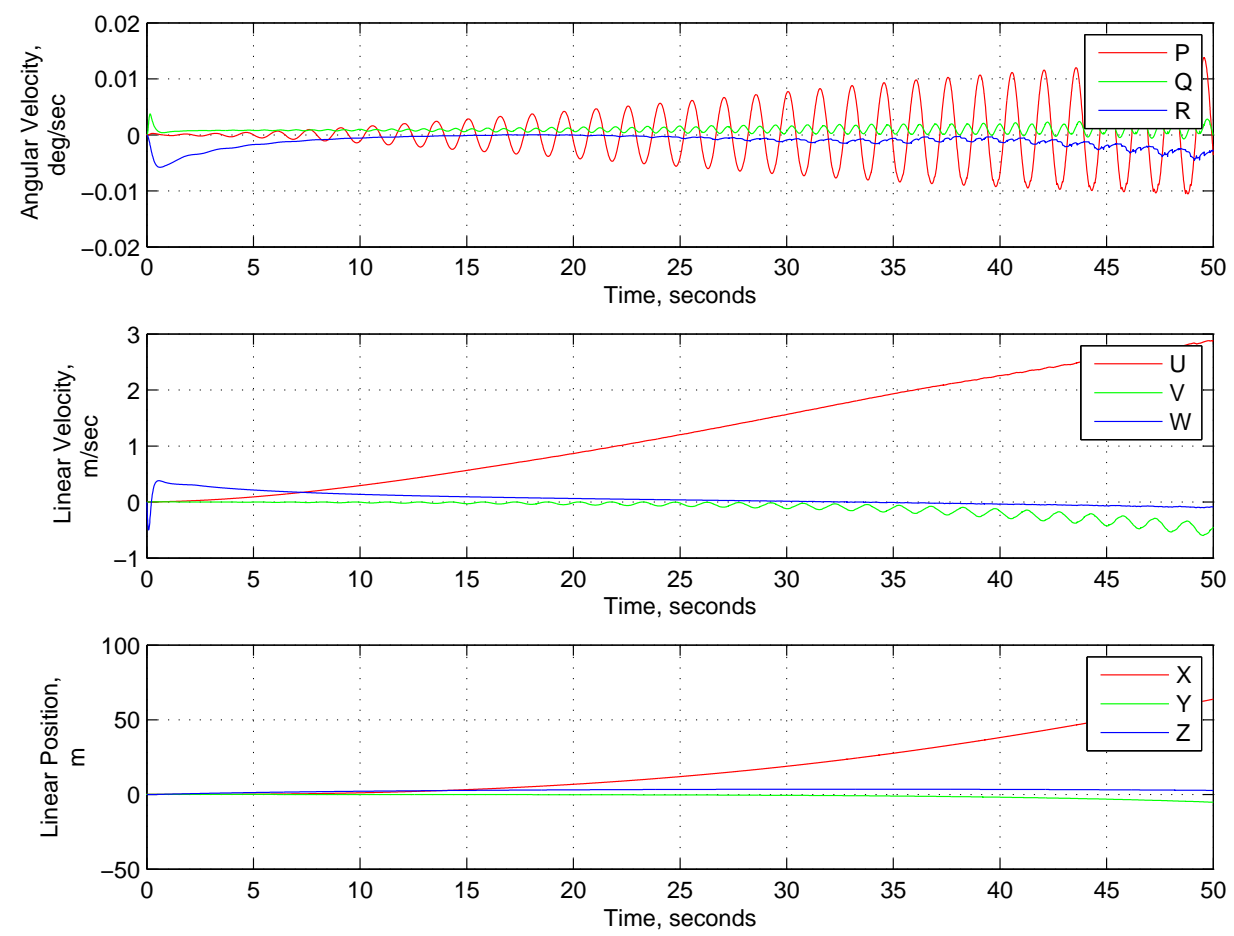

Figure 4.9: Time response for the quadrotor with a uneven mass

\subsubsection{Gamera: Uneven Rotor Pitch}

Figure 4.10 shows an initial positive vertical velocity, which decreases until the end of simulation. There is significant lateral displacement by the vehicle on the order of 75 meters. This will be clearly out of bounds as defined in competition rules. There is also the presence of high frequency-high amplitude angular oscillation about the lateral x-axis. These oscillations occur at approximately twice per rotor revolution, or $36 \mathrm{~Hz}$. Small amplitude oscillation with the same frequency begins in the lateral y-axis linear velocity, near the end of the simulation. The 
identified model for this simulation has two unstable eigenvalues. The stability of the identified system matches the divergence of the time response, visually. Geometric twist, mass and inertia perturbation simulations can serve to determine the building and balancing tolerances, as well as the assembly tolerances that will drive requirements in the design.
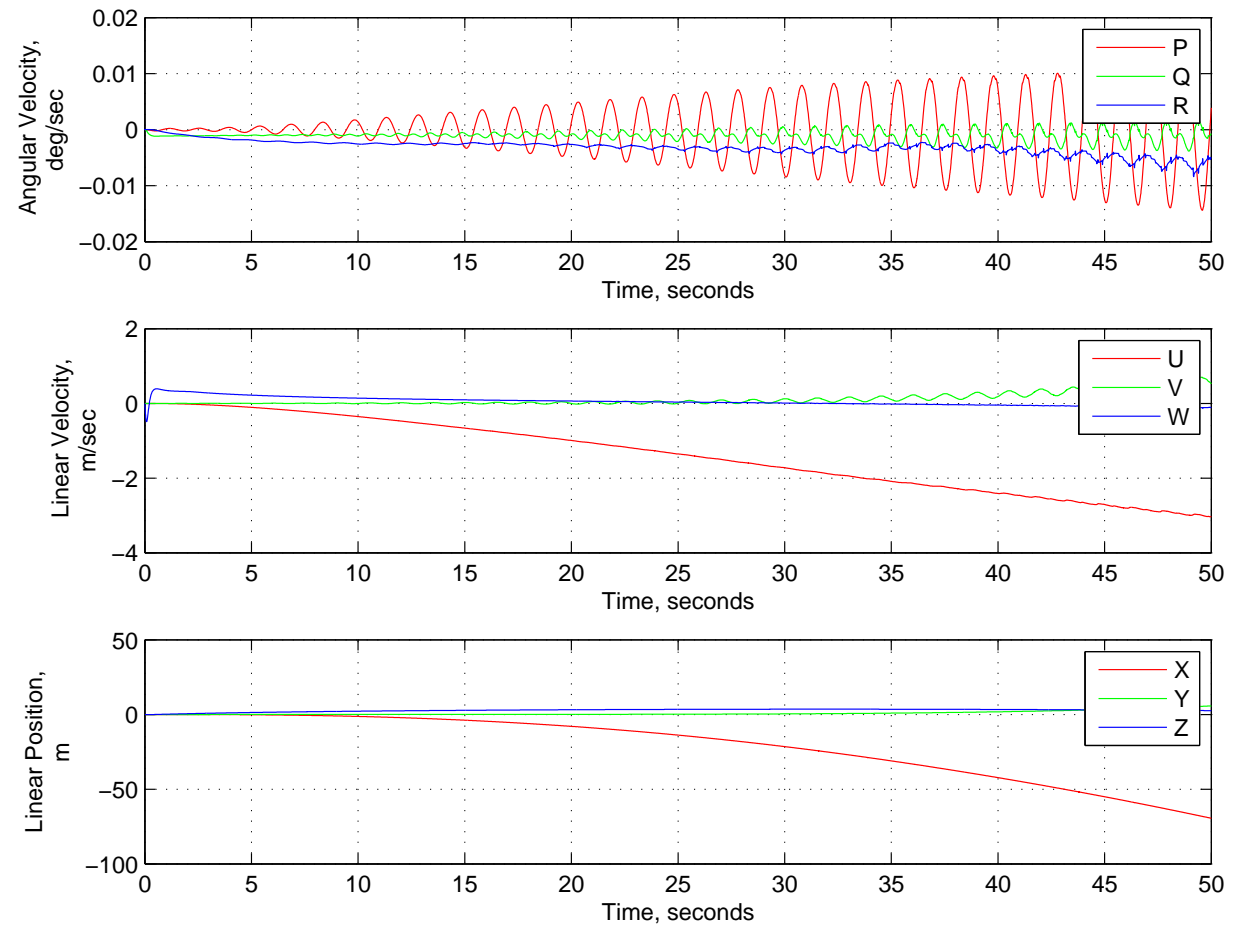

Figure 4.10: Time response for the quadrotor with a uneven geometric twist

\subsubsection{Gamera: Initial Angular Offset}

Figure 4.11 shows that the initial angular displacement of the vehicle generates the angular oscillation described in the previous simulations. This oscillation becomes relatively bounded by 20 seconds. High frequency noise is present in the vehicle yaw velocity. This high frequency noise is attributed to the aerodynamic model's method for handling reverse flow through stall. As the vehicle's angular velocity nears the rotor rotation speed, reverse flow regions are found near the 
tips of the blades. This effect will not be physically accurate. Operation of a $\mathrm{HPH}$ in this regime is not recommended. The linear velocity and displacement in the lateral direction begin to increase. The x-direction linear velocity stabilizes at approximately $5 \mathrm{~m} / \mathrm{s}$. Figure 4.12 shows the torque applied to each rotor in this simulation. The large oscillations in torque are caused by the high yaw rate. The rotors will experience high velocity, and reversed flow, depending on the rotor's azimuth. Actual flight test of these vehicles is not expected to reach this level of yaw velocity This analysis demonstrates important aspects of gear ratio and pilot selection.
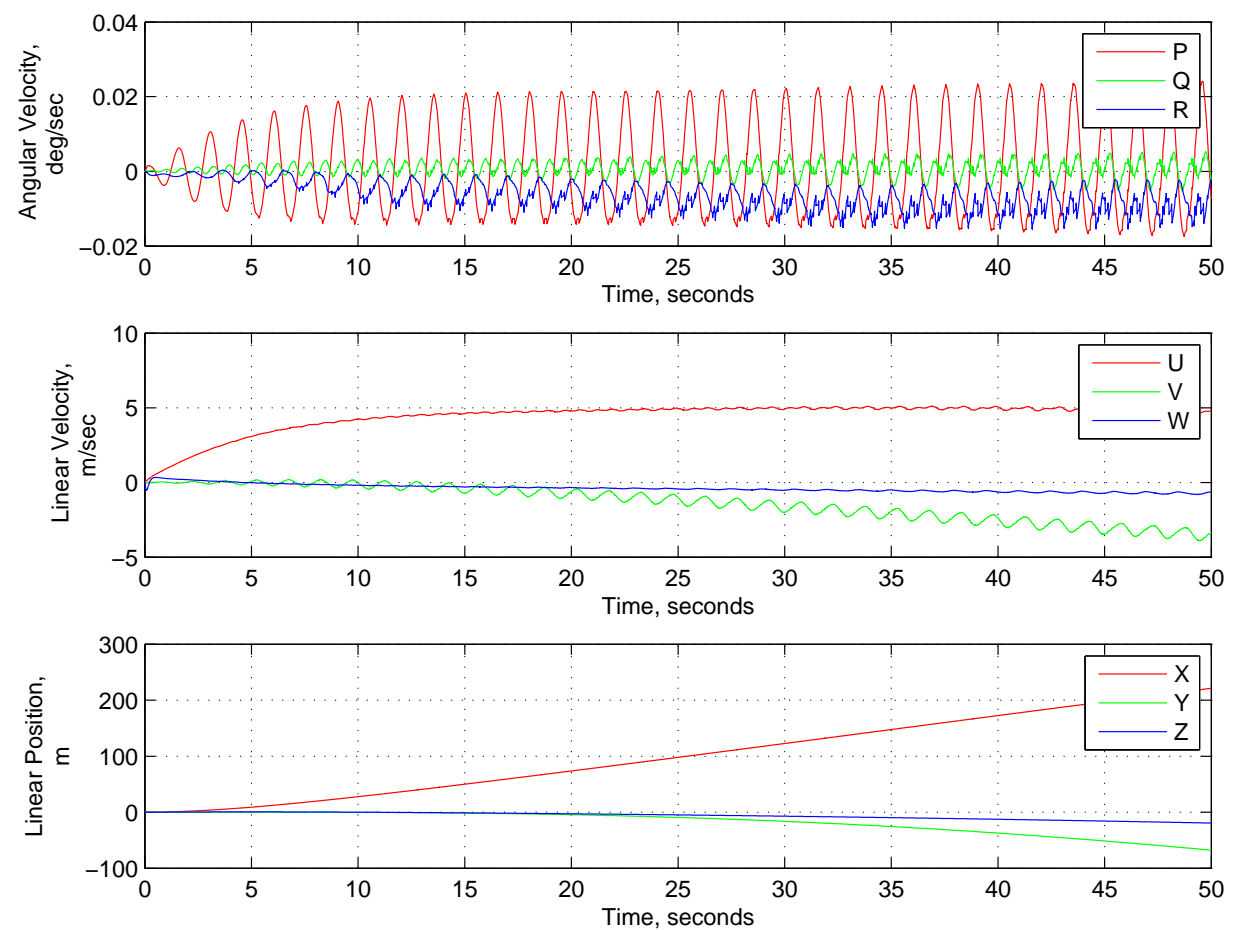

Figure 4.11: Time Response for the quadrotor with a initial angular displacement 


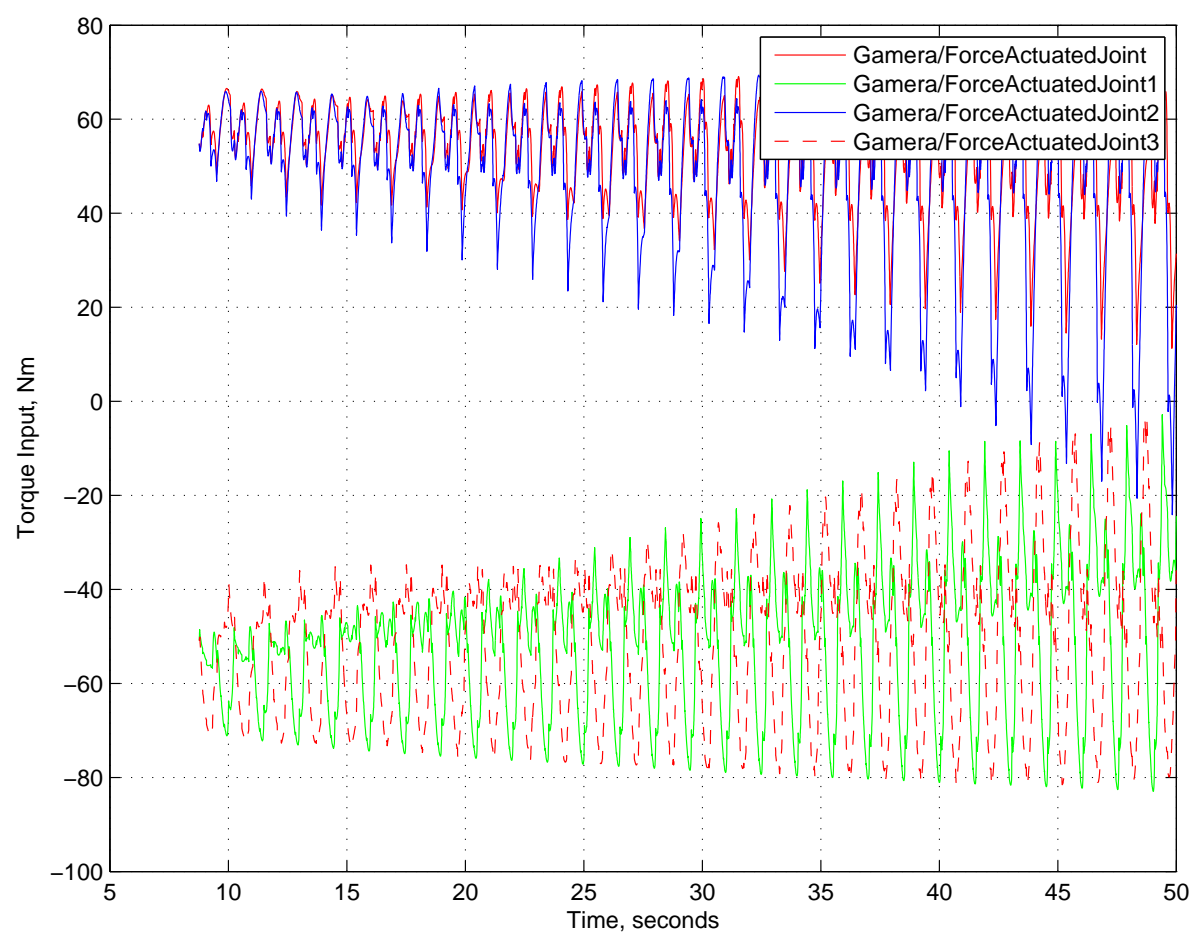

Figure 4.12: Torque Applied to rotors

\subsubsection{Gamera: Initial Linear Velocity}

Figure 4.13 shows that the carrier can recover initial linear velocity, and stabilize at a level attitude. The carrier in this simulation slowly climbs past an altitude of 4 meters at the end of the simulation. The vehicle does have a small sustained lateral velocity in both axis. This lateral velocity is also found when examining the video footage of the Yuri I flights in [55]. The identified model shows one unstable eigenvalue, which is due to the lateral linear displacement. 

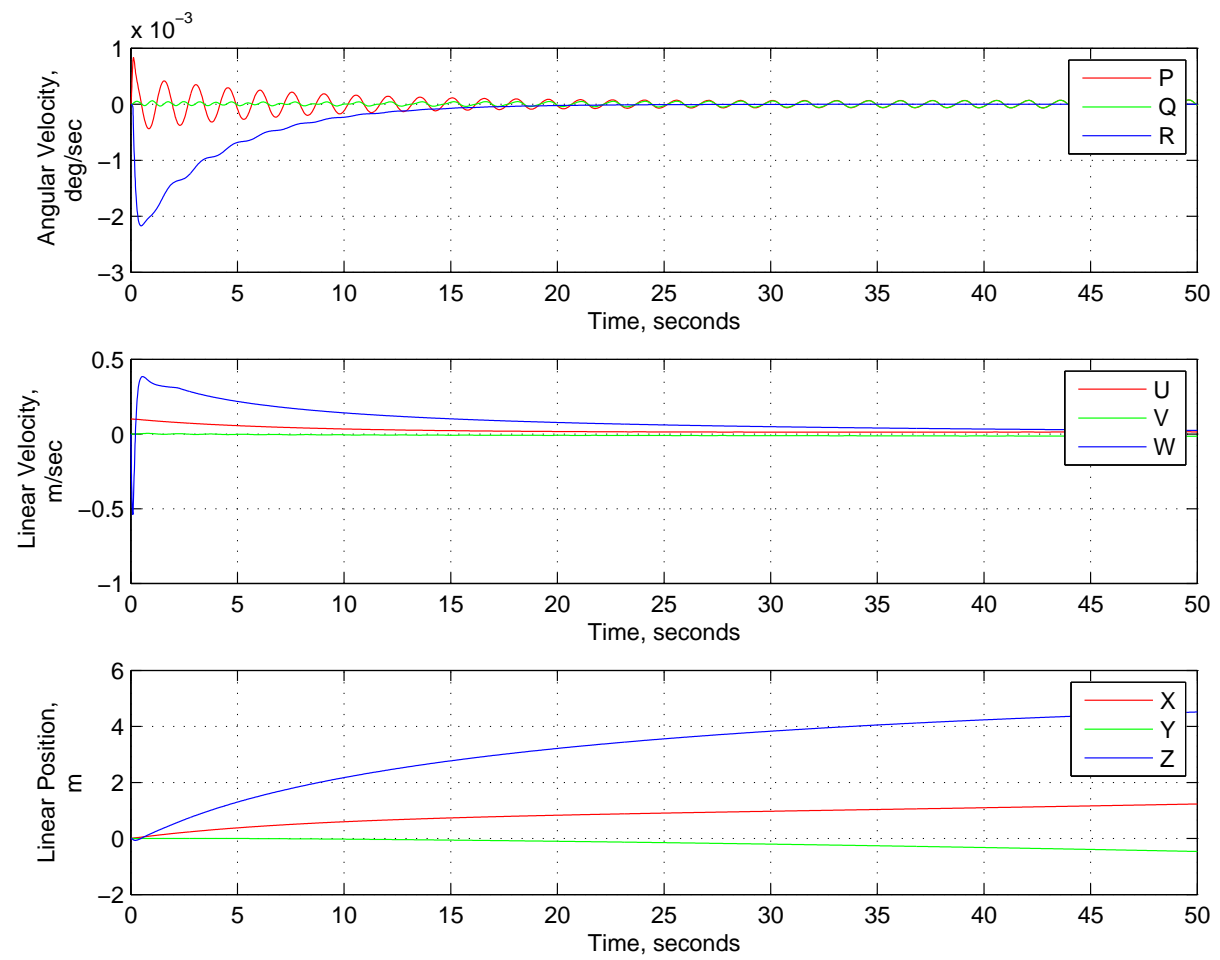

Figure 4.13: Time Response for the quadrotor with a initial linear velocity

\subsection{Parameter Studies}

Parametric studies of the quadrotor and tip-driven propeller HPH configurations are presented in this section. These parametric studies demonstrate this methods ability to rapidly trade configurations in the preliminary HPH design phase. The eigenvalue magnitudes are determined for changing values of HPH configuration parameters. The dihedral of each rotor blade will be varied in the quadrotor configuration. The dihedral angle is defined as zero when the rotor thrust axis aligns with the ground-fixed vertical axis. The dihedral angle is negative when all rotor thrust axes point toward the center of the vehicle and positive when then point away. The rotors and carrier will begin from rest. The simulation will run for 50 seconds. The vehicle is set at an initial angle of 5 degrees to induce a moderate dynamic response. Figure 4.11 shows the base time response for the quadrotor configuration with zero dihedral. 
Figure 4.14 show the change in the magnitude of each eigenvalue for changing dihedral angle. Stability is defined as an eigenvalue magnitude less than one. The vehicle is identified as stable at zero dihedral, which is corroborated by the perturbation study in the previous section. A trend is not easily found in this data due to the statistical methods used in system identification. The vehicle has an unstable response in all modes at high positive dihedral, which is accurate conceptually. The vehicle response has a trend to be stable a small positive dihedral angles. If this is a physical effect that can be validated in an experimental setup it could be used to the advantage of the design team in making the vehicle robust to initial offset angles, without losing a moderate amount of thrust.

The vehicle has a stable response at a negative dihedral angle of five degrees, which makes sense conceptually. The vehicle response is unstable at small negative dihedral angles, approximately -3 degrees, before stabilizing at -5 degrees. If this is a physical effect, it will be a pitfall for the designer who relies on conceptual wisdom for design. 


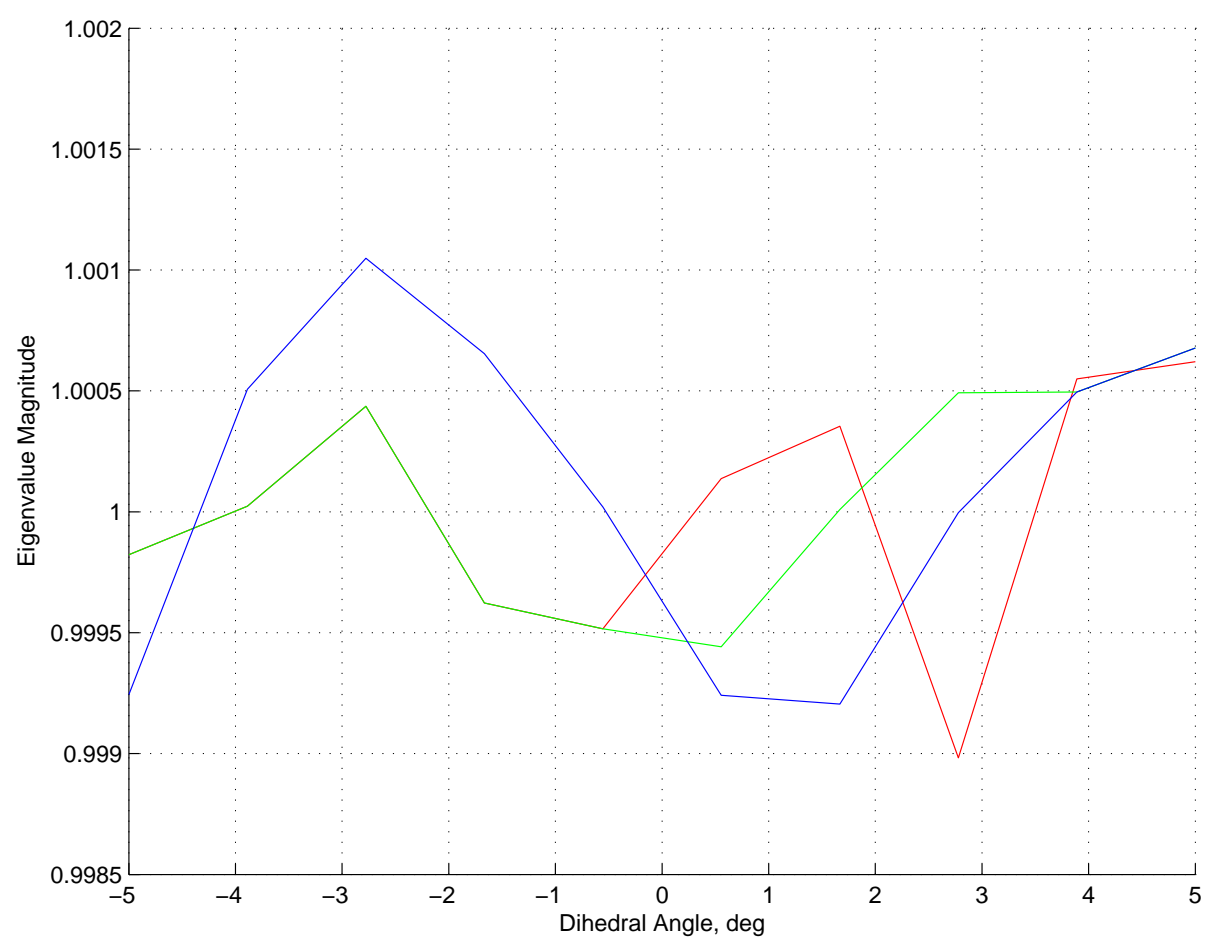

Figure 4.14: Eigenvalue Magnitude for change in Rotor Dihedral

Figure 4.15 shows the effect of moving the rotor from below the carrier to above the carrier in the tip-propeller driven main rotor configuration. This model was not perturbed, similar to the previous model, excitation present with the already unstable configuration. The model is unstable in every location, which is corroborated by the Da Vinci model base configuration time response shown in figure 4.5. The vehicle response is unstable in every configuration, with a trend for a less unstable system (Eigenvalue magnitude closer to 1) when the carrier below the rotor blade. This parameter study would be important in the HPH conceptual design phase. The reduced power required due to the rotor placement could be traded against the change to stability of the system. 


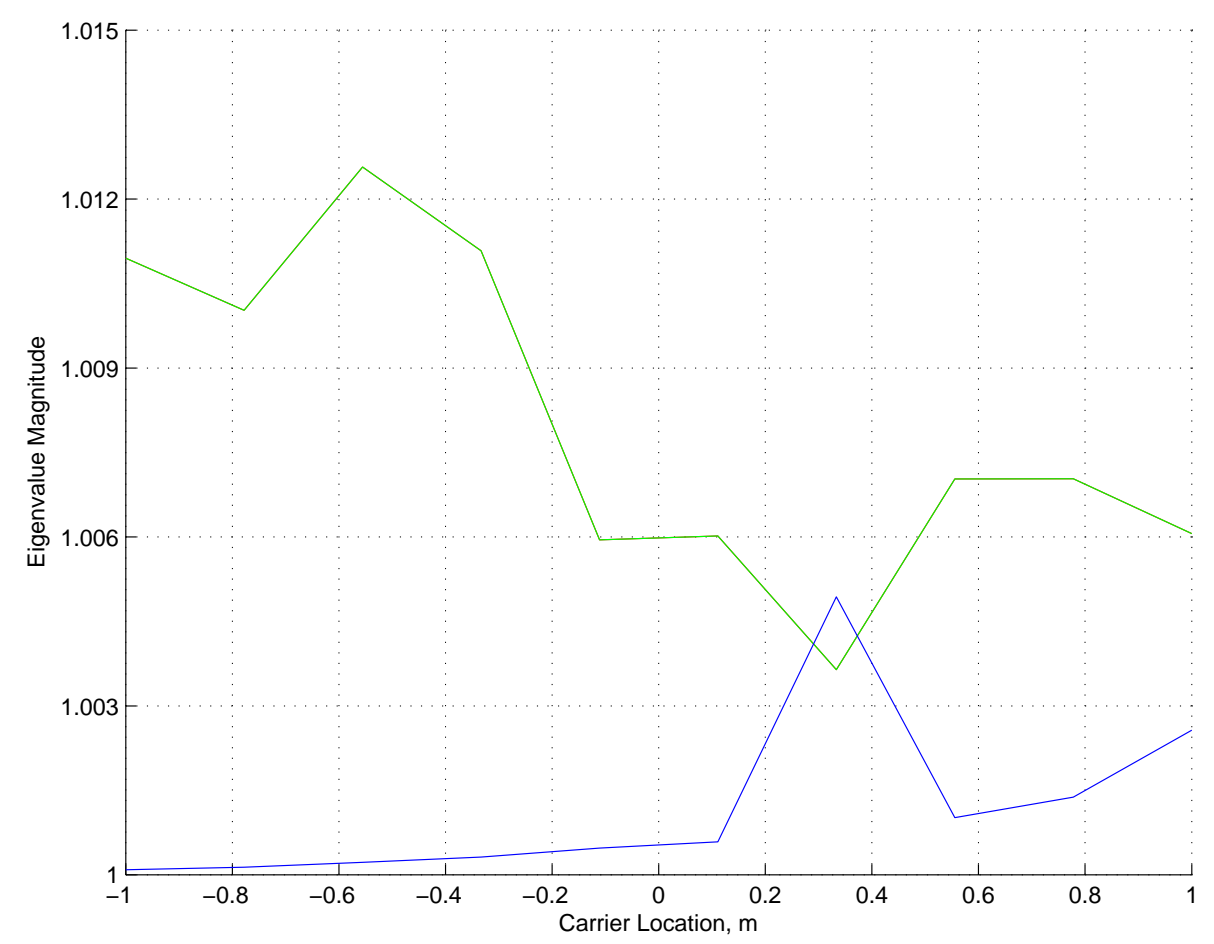

Figure 4.15: Eigenvalue Magnitude for changing carrier vertical location

\subsection{Summary}

The results chapter presented time responses and parametric stability studies for the quadrotor and tip propeller driven main rotor configurations. SimMechanics models were generated to mimic the Da Vinci III and Gamera I HPH configuration. The base configuration time response was presented for each configuration. The Da Vinci III simulation was unstable in its base configuration. The Gamera I simulation showed stability in it base configuration. The torque applied to the rotors was presented for the Gamera I base configuration. The Gamera I configuration was altered and the resulting dynamic time responses for the selected vehicle states were presented. The rotor mass, rotor inertia, and collective pitch were all altered on a single rotor of the quadrotor configuration. An initial angular vehicle attitude and initial linear velocity was also simulated and the time responses presented. 
The section 4.2 presented a parametric study of stability for the Gamera and Da Vinci configurations. For the Gamera configuration, the pointing angle, called the dihedral angle in this thesis, was varied for all rotors. The vehicle was set at an initial angle of 5 degrees as in the perturbation studies to excite the vehicle. Zero and negative 5 degrees of dihedral gave a stable response, while positive 5 degrees caused an unstable system response. The Da Vinci configuration's carrier location relative to the rotors was altered to determine its affect on the stability of the vehicle response. The response is unstable in all configurations, but does trend toward stability for a carrier far below the rotor blades. These trends are complex due to the system identification method used and the results of them are used to demonstrate the tool's abilities and are not meant to impinge on a design until the tool's models are verified and validated.

These results show that multi-body system simulation and system identification can be used to model the dynamic stability characteristics of the quadrotor and tip-propeller driven HPH configurations. These results can be extrapolated to encompass any HPH configuration that can be discretized into a multi-body system model. These results show that the method can be used to make HPH design decisions. 


\section{Chapter 5}

\section{Conclusion}

\subsection{Summary}

The Sikorsky prize has taunted inventors and hobbyists since 1980. Several groups have attempted to achieve the requirements of 3 meters, and 60 seconds. All attempts have failed to achieve both. There are three sides to this problem, Aerodynamics, Structures and Dynamic stability. HPH aerodynamics have been investigated using rotorcraft aerodynamic theory, wind tunnel tests, small scale models and full scale models. Every group reaches the conclusion that the rotors need to have an efficient airfoil design, with a large rotor that spin very slowly to reach peak power efficiency.

The structural design requirements of the HPH are derived from the aerodynamic analysis. These requirement show that the vehicle needs to have large rotors and low weight carbon fiber materials along with tension members and empty inner structure are used to achieve this type of structure in recent vehicles. The structure of the HPH is similar to the structure of other human powered aircraft. This similarity allows the application of the lessons learned

and best practices from the successful Daedalus project from MIT and Snowbird ornithopter from University of Toronto. 
Historical HPHs that achieve lift off have all experienced dynamic stability problems. The Da Vinci III achieved lift-off in 1989, but due to oscillation about a lateral axis it returned to the ground. The Gamera project has reached over 2 meters in vertical height and due to translation, lift was lost and the vehicle had a hard landing. The dynamic stability of the HPH has been research by three people. These three researchers did their work in the late 1980 during the Da Vinci program. Two of the researchers Totah and Patterson concluded that the Da Vinci configuration could be stable if a tip flap control system was implemented. This control system would actuate a tip flap based on the differential sensed tip height. The third researcher, Guttieri, reported the quasi-static assumption could not be used in the analysis of the Da Vinci configuration dynamics due to the difference in rotor rotation speeds and HPH response times. The Totah and Patterson report used the Quasi-steady assumption, thus rendering their analysis and conclusion as unusable. These reports show a lack of research in the area of dynamic stability for HPH.

The HPH problem sets requirements for aerodynamics, structures and dynamic stability. Aerodynamics and structures have been treated in numerous reports. The stability of the HPH cannot be easily approached by traditional helicopter dynamics methods and has not been properly treated. This thesis shows that the quasi-steady assumption cannot be used.

This thesis solves the problem of analysis of a HPH dynamics through use of multi-body system simulation, system identification and linear stability analysis theory.

Multi-body system simulation requires the development of models that represent discrete bodies and joints. A aerodynamic model is developed using combined blade element-momentum theory and implemented in the multi-body system. The multi-body system simulation generates a dynamic time response for a HPH configuration. The time response data is used to identify a linear discrete state space model through the process of System Identification. Linear Stability theory can then be applied to the state space model to determine its stability characteristics. These stability characteristics describe the stability properties 
of the time response data generated by the multi-body system simulation. This method can determine the stability properties of a HPH for specific flight conditions.

The multi-body system simulator is SimMechanics. SimMechanics is based on Simulink, a programming GUI. An interface to the SimMechanics GUI is developed for this thesis to allow for scripting of interactions with the multi-body system simulation. The interface uses object-oriented programming techniques to control data access and flow with SimMechanics.

The aerodynamic model implemented in SimMechanics is verified through a Richardson extrapolation and an effort is made to validate it with experimental data from UMD's Gamera project. The experimental data used didn't include experimental uncertainty data, and thus the aerodynamic model could not be validated.

The results of this thesis present a demonstration of the method for dynamic analysis of the Gamera quadrotor configuration. Time responses for the base quadrotor configuration and the base quadrotor configuration with varied properties are presented. The stability properties identified through system identification and linear stability analysis seemed to match the stability characteristics of the time response. Parametric studies are presented to demonstrate this methods ability to be used in a design capacity. The results show that this method of dynamics analysis can be used to determine the dynamic response and stability properties of a HPH configuration.

This thesis only presents the development and demonstration of this method and not results that should impinge on any HPH design efforts. The video footage of HPHs flight test provides confidence, that this method is correct for HPH application. 


\subsection{Future Work}

The tool needs improvement and focus in three areas: error propagation, system identification, and model development. The tool needs the capability to calculate the sensitivity of the SimMechanics model, which would involve a formal treatment of error propagation through complex systems. The system identification capabilities presented possess only preliminary abilities compared to the full field of knowledge that accompanies the process of system identification. It would be advantageous to assess the behavior and limits of the system identification method used.

Local aerodynamic moment should be added to the aerodynamic model. A moment is generated around the span wise axis, due to non-collocated aerodynamic center and center of gravity of an airfoil section. It is recommended that the aerodynamic moment be applied in concert with an elastic model allowing the rotor blade to twist about the span wise axis. This will enable the modelling of a vast number of dynamic modes present in physical helicopter blades, mainly feathering and feather-flap coupling. The aerodynamic moment can be calculated and applied in the same way as the lift is calculated and applied.

\subsubsection{Model Development}

The aerodynamic model will need to be re-evaluated and a structural and weights model developed, in order to capture the complex effects that are present in the HPH.

The structural model can easily be incorporated along side the aerodynamic model using the already discretized blade elements. The most basic level of the structural model will make the blades elastic and allow the rotors to flex during simulation. The more advanced for will have similar capabilities to that of a finite element analysis program, which will have the ability to measure stress and inform the designer of probable failure modes after simulation. Addition of a weights model will make a closed loop in the design, which will require iteration 
before simulation. This weight model, however, will enable more realistic trades of rotor radius.

The aerodynamic model presented here is validated for hover conditions, which is sufficient for the main rotors of the HPH. This is not sufficient for the high advance ratios that are experienced by the tip-propellers on the Da Vinci configuration, for example. Each model used needs to be verified and validated using experimental data. This, along with proper uncertainty analysis, will lead to confidence in the multi-body system simulation that is the ultimate goal of the tool. As the project progresses and physical components are built, the simulation model can be updated to present the most accurate dynamic response, which will intern continuously drive the vehicle design. Done properly, this method will reduce the cost and time required during the build and test phase of the project.

Slight alteration of the airfoil properties creates significantly different results. Lift and drag data for rotor blade should be measure in a non-rotating wind tunnel experiment to isolate true airfoil aerodynamic properties after rotor construction. 


\section{Bibliography}

[1] B. Berry, G. Bowen-davies, K. Gluesenkamp, Z. Kaler, J. Schmaus, W. Staruk, E. Weiner, and B. K. S. Woods, "Design Optimization of Gamera II : a Human Powered Helicopter," in American Helicopter Society 68th Annual Forum, 2012.

[2] G. Lehoux, "Project: Yuri I."

[3] University of Maryland, "Gamera II Photo Gallery," 2012.

[4] J. Langford, "The Daedalus Project : A Summary of Lessons Learned," in AIAA/AHS/ASEE Aircraft Design, Sysferns and Operations Conference, (Seattle, WA), 1989.

[5] T. J. Hawkins, Aerodynamic $\& 3$ power considerations for Human Powered Helicopter in Vertical Flight. PhD thesis, Stanford, 1996.

[6] J. J. Totah and W. Patterson, "Control of a Human-Powered Helicopter in Hover," Tech. Rep. November, 1988.

[7] A. Bramwell, G. Done, and D. Balmford, Helicopter Dynamics. ButterworthHeinemann, 2nd ed., 2001.

[8] G. Rouleau and S. Popinchalk, "Modeling Mechanical Systems: The Double Pendulum," 2009.

[9] AHS, "AHS Igor I. Sikorsky Human Powered Helicopter Competition," 2012. 
[10] S. Larwood and N. Saiki, "Aerodynamic Design of the Cal Poly Da Vinci Human Powered Helicopter.pdf," in American Helicopter Society Vertical Lift Aircraft Design Conference, 1990.

[11] K. Colvin, "Da Vinci III flight: Human Powered Helicopter," 2009.

[12] W. Staruk, B. Woods, B. Berry, and G. Bowen-davies, "DESIGN AND FABRICATION OF ULTRA-LIGHTWEIGHT COMPOSITE STRUCTURES FOR THE GAMERA HUMAN- POWERED HELICOPTERS," 2011.

[13] P. Ridden, "Gamera II human-powered helicopter flight record confirmed by NAA," gizmag, Aug. 2012.

[14] J. Schmaus, B. Berry, G. Bowen-davies, B. Bush, C. Friedman, M. Gilad, A. Sridharan, W. Staruk, and B. Woods, "Design and Development of Gamera : A Human Powered Helicopter from the University of Maryland," in American Helicopter Society 68th Annual Forum, 2011.

[15] A. Naito, "Review of Developments in Human-Powered Helicopters," International Human Powered Vehicle Association, vol. 9, no. 2, 1991.

[16] TeamGameraHPH, "Gamera II HPH 8.6 ft (2.6 m) Altitude Flight and Hard Landing," 2012.

[17] M. Gilad, Evaluation of Flexible Rotor Hover Performance in Extreme Ground Effect. Master's thesis, University of Maryland, 2011.

[18] J. R. Cruz, "Weight Analysis of The Daedalus Human Powered Aircraft," Technical Soaring, Jan. 1990.

[19] J. R. Cruz and M. Drela, "Structural Design Conditions for Human Powered Aircraft," in OSTIV, (Wiener Neustadt, Austria), 1989.

[20] R. B. Sullivan and S. H. Zerweckh, "Flight Test Results for the Daedalus and Light Eagle Human Powered Aircraft," tech. rep., Massachusetts Institute of Technology, Cambridge, MA, 1988. 
[21] M. Drela, "Low-Reynolds-number airfoil design for the M.I.T. Daedalus prototype- A case study," Journal of Aircraft, vol. 25, pp. 724-732, Aug. 1988.

[22] P. Lissaman, H. R. Jex, and P. Maccready, "Aerodynamics of Flight at Speeds under $5 \mathrm{~m} / \mathrm{s}$," tech. rep., 1978.

[23] S. Finberg and R. B. Sullivan, "The flight-control system for the Daedalus Human Powered Aircraft," AIAA, vol. 218, pp. 83-90, May 1968.

[24] H. R. Jex and D. G. Mitchell, "Stability and Control of the Gossamer Human-Powered Aircraft by Analysis and Flight Test," tech. rep., Systems Technology, Inc, Hawthorne, CA, 1982.

[25] T. Reichert and C. D. Robertson, "Aircraft Overview: Snowbird HumanPowered Ornithopter," tech. rep., University of Toronto Institute for Aerospace Studies, Toronto, Canada, 2010.

[26] F. E. Mehler, The Structural Testing and Modification of a full-scale Ornithopter's Wing Spars. PhD thesis, University of Toronto, 1997.

[27] C. D. Robertson, Structural Characterization, Optimization, and Failure Analysis of a Human-Powered Ornithopter. PhD thesis, University of Toronto, 2009.

[28] J. D. Delaurier, "The development of an efficient ornithopter wing," tech. rep., University of Toronto Institute for Aerospace Studies, Downsview, Ontario, Canada, 1993.

[29] R. F. Larijani, A Non-Linear Aeroelastic Model for the Study of FlappingWing Flight. PhD thesis, University of Toronto, 2000.

[30] J. D. Delaurier, "An Aerodynamic Model For Flapping-Wing Flight," The Aeronautical Journal of the Royal Aeronautical Society, 1993.

[31] AHS, "AHS Congratulates AeroVelo for Human Powered Helicopter First Flight!," 2012. 
[32] R. Guttieri, "Human Powered Helicopter Stabilization," tech. rep., Cal Poly San Luis Obispo, San Luis Obispo, 1988.

[33] AHS, "Upturn Human Powered Helicopter Flies!," 2012.

[34] G. D. Padfield, Helicopter Flight Dynamics. Oxford, UK: Blackwell Publishing Ltd, 1st ed., Jan. 2007.

[35] W. Schiehlen, "Multibody System Dynamics : Roots and Perspectives," Multibody System Dynamics, vol. 1, pp. 149-188, 1997.

[36] R. von Schwerin, MultiBody System Simulation. 1999.

[37] M. Zaeh and D. Siedl, "A New Method for Simulation of Machining Performance by Integrating Finite Element and Multi-body Simulation for Machine Tools," CIRP Annals - Manufacturing Technology, vol. 56, no. 1, pp. 383386, 2007.

[38] Z. Zhao and G. Ren, "Multibody Dynamic Approach of Flight Dynamics and Nonlinear Aeroelasticity of Flexible Aircraft," AIAA Journal, vol. 49, no. $1,2011$.

[39] I. Argatov, "Development of an asymptotic modeling methodology for tibiofemoral contact in multibody dynamic simulations of the human knee joint," Multibody System Dynamics, vol. 28, pp. 3-20, Nov. 2011.

[40] J. Cuadrado, D. Dopico, J. a. Perez, and R. Pastorino, "Automotive observers based on multibody models and the extended Kalman filter," Multibody System Dynamics, vol. 27, pp. 3-19, Apr. 2011.

[41] P. Van Overschee and B. De Moor, Subspace Identification for Linear Systems. Kluwer Academic Publishers, 1996.

[42] E. A. Morelli and V. Klein, "Application of System Identification to Aircraft at NASA Langley Research Center," AIAA Journal of Aircraft, vol. 42, no. 1, 2005. 
[43] W. Johnson, Helicopter Theory. Dover Books on Aeronautical Engineering Series, Dover Publications, 1994.

[44] A. Filippone, "On the Possibility of Human-Powered Vertical Flight," Journal of the American Helicopter Society, vol. 52, no. 4, p. 371, 2007.

[45] R. L. Bielawa, Rotary Wing Structural Dynamics and Aeroelasticity. Reston, VA: AIAA, 2nd ed., 2006.

[46] J. G. Leishman, Principles of Helicopter Aerodynamics. 2 ed., 2006.

[47] R. Steijl and G. N. Barakos, "Computational Study of Helicopter RotorFuselage Aerodynamic Interactions," AIAA Journal, vol. 47, pp. 2143-2157, Sept. 2009.

[48] M. Gilad, I. Chopra, and O. Rand, "Performance Evaluation of a Flexible Rotor in Extreme Ground Effect," 2012.

[49] J. Schmaus, B. Berry, and W. Gross, "Experimental Study of Rotor Performance in Deep Ground Effect with Application to a Human-Powered Helicopter," in American Helicopter Society 68th Annual Forum, (Fort Worth, Texas), 2012.

[50] W. J. Rugh, Linear Systems Theory. Prentice-Hall, 2nd ed., 1996.

[51] MathWorks, "Identifying State-Space Models :: Linear Model Identification ( System Identification Toolbox )."

[52] W. L. Oberkampf and T. G. Trucano, "Verification and Validation in Computational Fluid Dynamics," Tech. Rep. March, Sandia National Laboratories, 2002.

[53] I. B. Celik, U. Ghia, P. J. Roache, and C. J. Freitas, "Procedure for Estimation and Reporting of Uncertainty due to Discretization in CFD Applications," tech. rep., West Virgina University, Morgantown, West Virginia.

[54] W. L. Oberkampf, "Verification and Validation in Computational Simulation," 2004. 
[55] NHK, "Causing The Wind: The Human Helicopter," 1994.

[56] V. Chudnovsky, A. Mukherjee, J. Wendlandt, and D. Kennedy, "Modeling Flexible Bodies in SimMechanics," tech. rep., 2006. 


\section{Appendix A}

\section{Custom Library Blocks}

The custom library block are used to take advantage of modularization. The hierarchy of the custom library blocks is shown below in figure A.1.

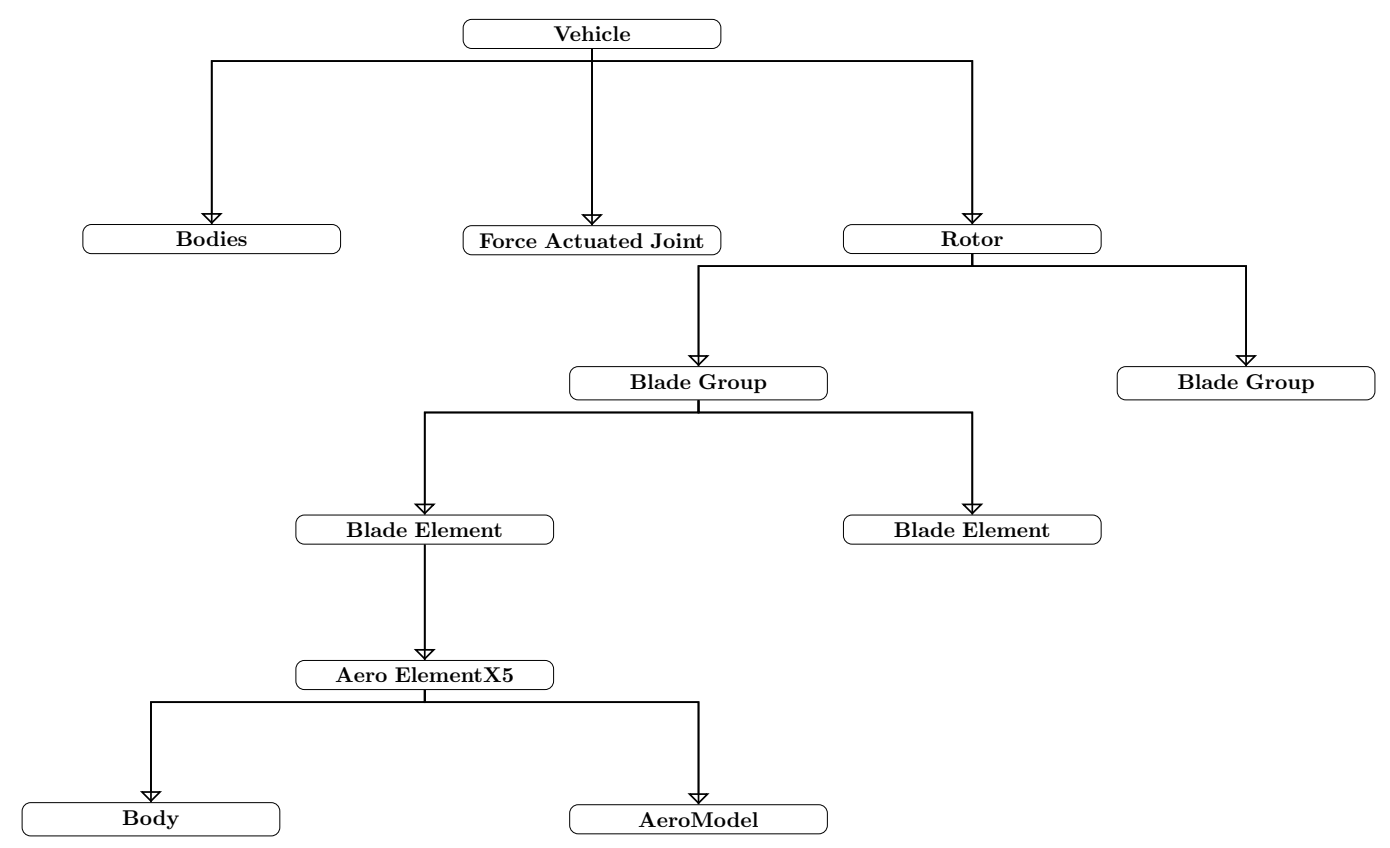

Figure A.1: Class Diagram 


\section{A.1 Aerodynamic Model}

The theory for the aerodynamics model is discussed in the main body of the thesis above, but the actual implementation was a little less straight forward. The aerodynamics model is a subsystem that has both output and input connectors to the body which it is acting on. The AeroModel also has input for the current dimensional coordinate where the element is located. This allows the user to specify non-constant geometric properties along the span of the rotor blade. There are two major subsystems in the AeroModel, Velocity Modification, and AngleWForces. Velocity Modification uses the sensed linear and angular velocity, local pitch, and lift curve slope to determine the local inflow ratio and whether the blade is stalled due to reversed flow. The AngleWForces subsystems uses the local blade characteristics and velocities to determine the dimensional lift and drag forces that are acting at this blade section. The inflow angle, $\phi$ is also calculated, and is used to rotate the wind axes into the local blade axes. The three element column vector of forces is then sent to the body actuator which will change the Simulink signal into a SimMechanics signal to actuate the Aero element.

The AeroElement is the smallest complete body element, and contains a body block, and a AeroModel subsystem that actuates it.

The BladeElement is a grouping of five AeroElements. The BladeGroup is a set of BladeElements. In the custom library, there are several BladeGroups with the number in the name designating the number of AeroElements that are contained within the set. The Blade group is meant to represent a complete rotor blade. Two blade groups and a hub body make up a the rotor block. The rotor block is a masked subsystem that contains a bus creator to package the user-defined rotor properties for use in the AeroModel. 


\section{A.2 Force actuated joint}

The Force Actuated joint is generated to model a powerplant used to run the $\mathrm{HPH}$. In this case, we are looking to run the rotor at a command rotational speed. To achieve this, a feedback system was implemented where the rotational speed of the joint is sensed and using a PID controller actuates the joint. This joint uses a subsystem mask for ease of interfacing. The several quantities are output for uses in exterior calculation. The torque input is used for System identification. The reaction forces and torques are used to calculate the power and thrust. 


\section{Appendix B}

\section{How to's}

This chapter will run through an example of building a Co-axial HPH configuration. The specifications this example are given in Table B.1. This tutorial will assume a basic level of familiarity with Simulink and MATLAB. It will also require the use of the Custom library blocks file that was detailed in the last chapter. Figure B.1 outlines the steps for building and simulating a HPH SimMechanics model. 


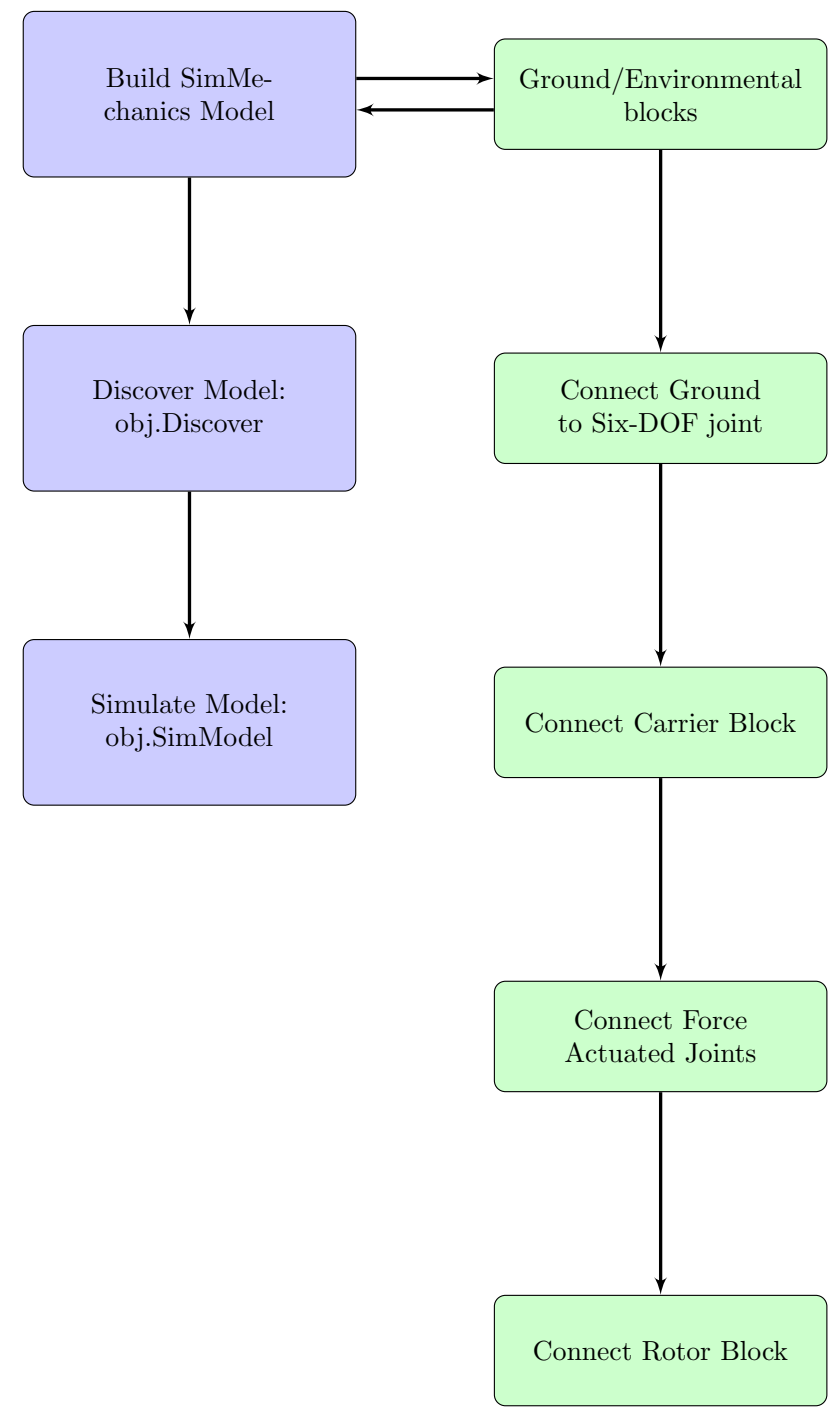

Figure B.1: How to: 
Table B.1: Coaxial Helicopter Characteristics

\begin{tabular}{|l|l|}
\hline Characteristic & Co-Axial rotor \\
\hline Rotor Radius $(\mathrm{m})$ & 6.5 \\
\hline \# Elements per blade $(-)$ & 10 \\
\hline Rotation Speed $(\mathrm{rad} / \mathrm{s})$ & 2.09 \\
\hline Blades \# per rotor $(-)$ & 2 \\
\hline Tip Reynold's \# $\left(\times 10^{5}\right)$ & 9 \\
\hline
\end{tabular}




\section{B.1 Build a Compliant SimMechanics Model}

In this section, a SimMechanics model, that is compliant with the interface, will be built. The basic block that will be needed in any SimMechanics model are the Ground, Machine Environment and a joint to connect the ground to the vehicle. The carrier body block is connected to the ground via a 6-dof custom joint. The carrier body block will represent all mass and inertia that is not represented by the rotors. In the carrier body block, the coordinate systems need to be configured such that all coordinate systems are relative to CS1. Since we are using two rotors, the Carrier Body block will have two Coordinate systems that are located at the rotor hub. Next, two Force Actuated Joints are added to actuate the two rotors. Attached to the actuators are the rotor subsystems. The mask properties for the rotors, and force Actuated Joints, need to be set. The properties used in this example are given in Table B.2. For this example, the Center spring stiffness, Center Spring Damping, Initial Frequency, Final Frequency, Chirp Signal Gain, and Sample Time will be ignored. The final built model is shown in figure

Table B.2: SimMechanics Coaxial Model characteristics

\begin{tabular}{|l|l|l|l|}
\hline Property & Value & Property & Value \\
\hline Rotor Radius $(\mathrm{m})$ & 6.5 & Rotation Speed $(\mathrm{rad} / \mathrm{s})$ & 2.09 \\
\hline \# Elements per blade $(-)$ & 10 & Chord Profile $(\mathrm{m})$ & 1 \\
\hline Rotation Speed $(\mathrm{rad} / \mathrm{s})$ & 2.09 & Pitch Profile, $\mathrm{deg}$ & $5 *$ ones $(1, \mathrm{Nb})$ \\
\hline $\mathrm{Nb}(-)$ & 2 & Joint Axis of Action & {$\left[\begin{array}{ll}0 & 0\end{array}\right]$} \\
\hline$C_{l \alpha}$ & ones $(1, \mathrm{Nb}) * 6.45$ & Rotation Speed $(\mathrm{RPM})$ & 20 \\
\hline Kaero & $.013 *$ ones $(1, \mathrm{Nb})$ & Signal Type & 4 \\
\hline$C_{d o}$ & $.01216 *$ ones $(1, \mathrm{Nb})$ & & \\
\hline
\end{tabular}




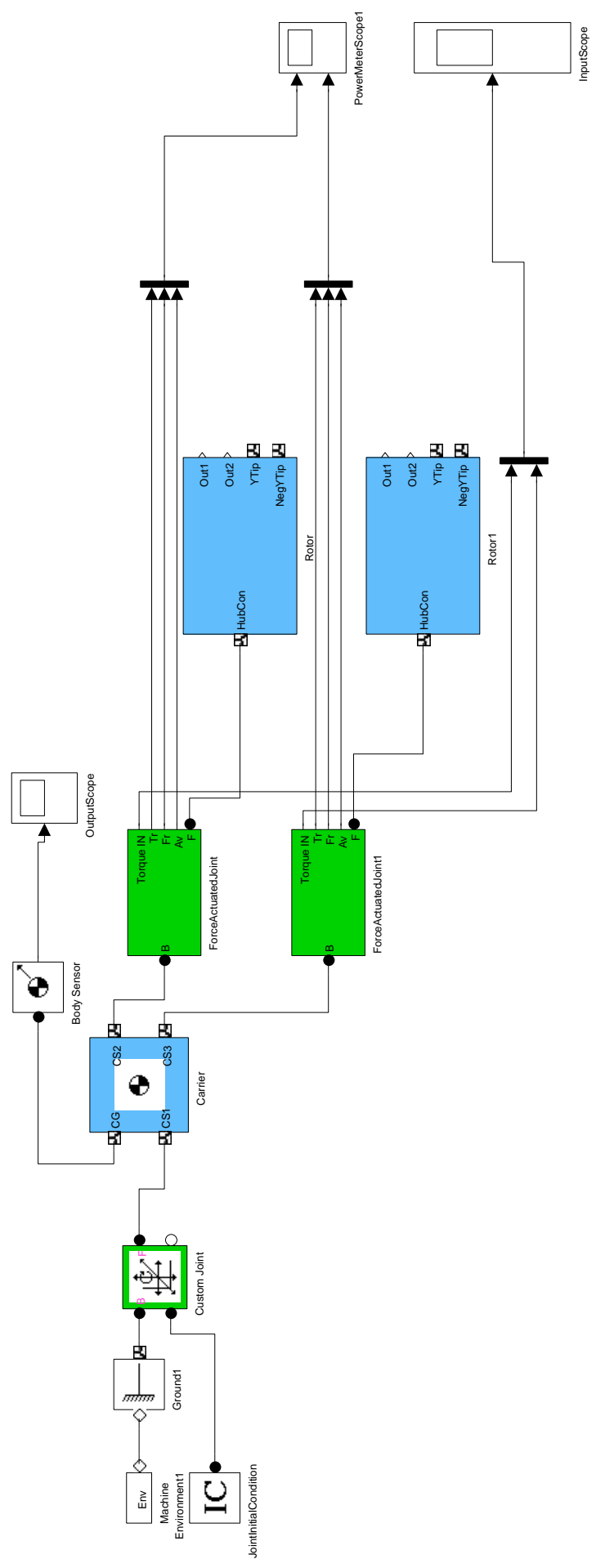

Figure B.2: SimMechanics Model of CoAxial HPH configuration 


\section{B.2 Use the Model Discovery Class}

During instantiation of the object, only the model name is required. The action of opening and accessing the model begins in the next step with the Discover method. This method will perform the function of exploring the designated model and looking for keywords that correspond to the general layout of the HPH. The current list of keywords are as follows: Carrier, ActuatedJoint, Prop, Rotor, Scope, InitialCondition. These blocks will be assigned a Parameter class object which will hold all of the block's usable parameters as well as their access methods. Attached to each parameter class object are event listeners that will trigger the simulation to run if a parameter is changed. This gives the designer instant feedback about a single change to the design. The ParameterVecIN method will change all or some of the block parameters, which is useful for multi-variable optimization.

The ModelDiscovery object contains the following list of key properties: $P a$ rameterStruct, Scopes, SysIDChar, FOM. ParameterStruct organizes all the parameter objects in a structure. ParameterList is a duplicate list that contains all the same Parameter objects. Since the objects are all handles, changing one will change the other instance. The scopes property is a structure of scopedata objects that are organized based on the name of the scope they were taken from. Keywords for scopes are Input, Output, Carrier and PowerMeter; these are case insensitive. The SysIDChar contains a structure that holds information about the identified system that is found during the system identification process. Finally, the FOM property contains a structure that holds any important figures of merit. Currently, FOM contains only the metrics for total steady state power and total steady state thrust. These are tuned to the Gamera configuration. The Scopedata class is also a custom class that will use the structure output to the workspace from Simulink to build a object to contain the data. The scopedata object contains methods that allow it to easily plot and label the data that it contains. 


\section{B.3 Generate Time Response Plots}

The simulation of the model can be executed using the SimModel method. This method uses the sim function to execute simulation on the specified Simulink model, but this method also captures, organizes and processes the scope data. The SimModel method also initiates the system identification process, on the model Input and Output data available. A plot of the time response of this example is given in figure B.3. Once the SimModel method has executed, the ModelDiscovery object is populated with information from the Scopes, Figures of Merit and the identified system. This process can be repeated to perform multi-variable optimization or quantitative trades studies.
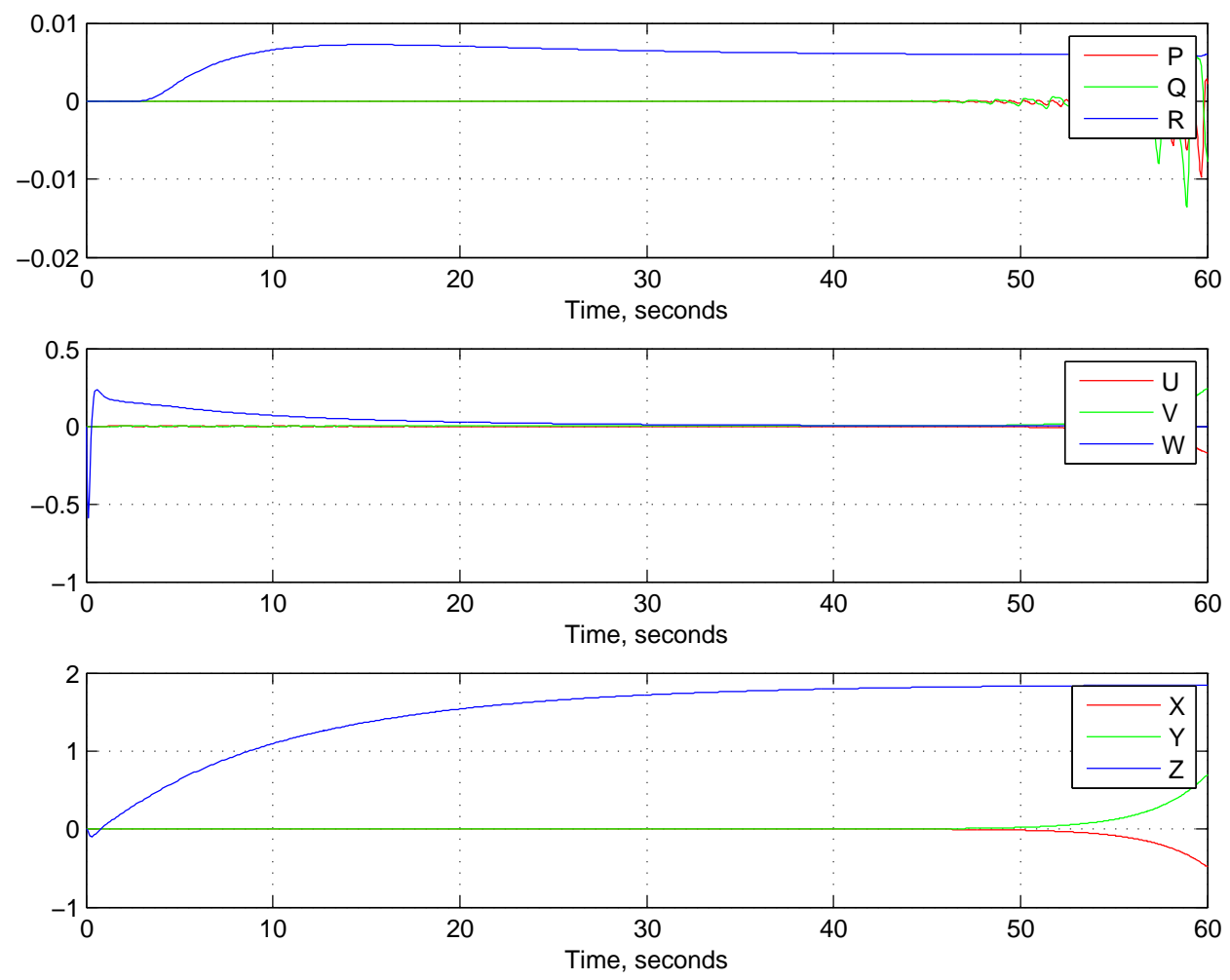

Figure B.3: Carrier Time Response 


\section{B.4 Add Structural Model}

A structural model will be necessary to get a realistic dynamic representation of the HPH. Currently, the rotor subsystem block uses weld joints in-between each Aero Element. If a joint with some degree of freedom,(e.g. flap, lag, and/or feather) is used instead, this will allow the elements to move relative to each other. The system can mimic the elasticity of a beam, if a spring-damper block is attached to the joint. This work is done in [56]. A example SimMechanics Blade element block with elasticity implemented is shown in figure B.4, where the Revolute $S D$ block is a subsystem that contains the revolute joint with attached spring damper. This subsystem is masked to allow input of the Spring and damper constants. A significant amount of computational power will be required if all 3 degrees of freedom are used. It is suggested that a lower order stiff solver be used to decrease the computation time required for the Aeroelastic model.

The center spring assumption can be used as a simplification to a fully elastic beam. In this model, only the center most joint will have any degrees of freedom, with the other joints being welded.

A elastic rotor subsystem can be made into a library block, once the elasticity model has been choosen. 


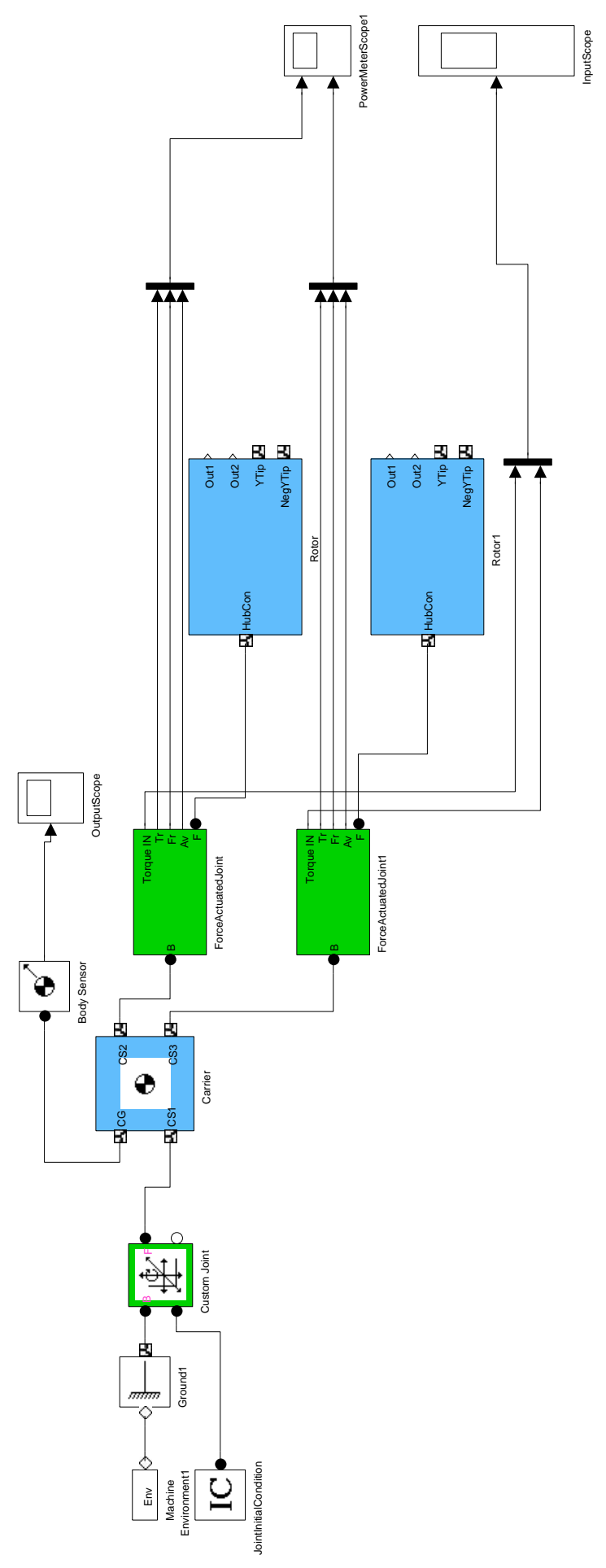

Figure B.4: SimMechanics Model of Elastic Blade element 


\section{B.5 Add Powerplant Model}

A realistic powerplant model can be built to mimic effects of rider power saturation, rider endurance limitations, gear ratios, and side-to-side wobble. This model would build-on/replace the Force Actuated joint that is currently used. This model would be used for the dynamic response, where a commanded rotation speed will be more useful for designing and comparing complete configurations. 